\title{
Welche Qualität macht den Unterschied?
}

\author{
Plastisch-Ästhetische Nasenchirurgie \\ Which Quality Makes the Difference?
}

\author{
Cosmetic Rhinoplasty
}

\section{(ㄷ) (1) $(8) \Theta$}

Autor

Andreas Dacho

Institut

Praxis für Plastische und Ästhetische Chirurgie, ATOS Klinik

Heidelberg

Schlüsselwörter

Nasenchirurgie, Rhinoplastik, Qualität, plastisch-ästhetische Chirurgie

Key words

Rhinoplasty, quality, cosmetic surgery

Bibliografie

DOI https://doi.org/10.1055/a-1012-9407

Laryngo-Rhino-Otol 2020; 99: S165-S194

(c) Georg Thieme Verlag KG Stuttgart · New York

ISSN 0935-8943

Korrespondenzadresse

Priv.-Doz. Dr. med. Dr. med. habil. Andreas Dacho

Praxis für Plastische und Ästhetische Chirurgie

ATOS Klinik Heidelberg

Bismarckstr. 9-15

D-69115 Heidelberg

Tel.: + 49 (0) 6221/9832900, Fax: +40 (0) 6221/9832929

nase@atos.de

\section{ZUSAMMENFASSUNG}

In kaum einem anderen Bereich ist das Ergebnis so offensichtlich, wie bei der plastisch-ästhetischen Nasenchirurgie. Umsomehr spielen sowohl die Qualität des Eingriffs und im Bereich der Ästhetik die Qualität des Ergebnisses eine herausragende Rolle. Hierbei sind die Erwartungen und der Anspruch des Operateurs nicht immer deckungsgleich mit der des Patienten. Des Weiteren haben Kostenträger, im Falle von Kombinationsoperationen, einen eigenen Fokus zum Thema Qualität, der häufig mit Wirtschaftlichkeit verwechselt wird. Objektive Kriterien spielen für den Arzt eine wesentliche Rolle, wobei für die Patienten sehr häufig weiche Kriterien von wesentlicher Bedeutung sind. Dies ist umso diffiziler, da Schönheit im Auge des Betrachters liegt und mit funktionellen Kriterien, wie Atmung und Riechen vereinbar sein müssen. In der Vielzahl von Qualitätskriterien fällt es dem Laien schwer die richtige Arztwahl zu treffen, insbesondere angesichts unzähliger Siegel, Zertifkate und des Einflusses des Internets bzw. von Social Media. Aber auch der Chirurg muss aus einer Vielzahl von Kongressen, Kursen und Symposien herausfinden, welche dieser Veranstaltungen eine bestimmte Mindestqualität garantiert. Trotz einer enormen Veränderung und Verfeinerung der rhinochirurgischen OP-Techniken im Rahmen der letzten Jahrzehnte, wird die Ergebnisqualität seitens der Patienten konstant vor die Prozedurqualität gestellt. Dieses Referat versucht eine Standortbestimmung möglichst objektivierbarer Qualitätsindikatoren in Kombination mit unterschiedlichen Anforderungen und Standpunkten zum Thema Qualität - aus Sicht des Arztes, des Patienten und des Kostenträgers - zu beleuchten und diese im Spiegel sich verändernder OP-Techniken der plastisch-ästhetischen Nasenchirurgie zu betrachten.

\section{ABSTRACT}

In hardly any other field outcome is more obvious as in plasticaesthetic nasal surgery. Both the quality of the procedure and the quality of the result play an outstanding role in the arena of aesthetics. Expectations and interests of the surgeon are not always congruent with those of the patient. Furthermore, payers, in the case of combination surgery, have their own focus on quality, which is often confused with economy. Objective criteria play an essential role for the physician, with soft criteria being very important for patients. This is much more difficult because beauty is in the eye of the beholder and must be compatible with functional conditions such as breathing and smelling. In the variety of quality standards, it is difficult to make the right choice of surgeon for the non-professional, especially in the face of countless seals, certificates and the influence of the internet and social media. But even the surgeon has to ascertain a minimum level of quality from a large number of congresses, courses, and symposia. Despite a tremendous change and refinement of the rhinosurgical techniques over the past decades, quality of the outcome is constantly being prioritized for process quality by the patients. This paper attempts to define most objective quality indicators in combination with different requests and aspects on quality through the eyes of physicians, patients and payors and tries to asses these facts in the context of changing surgical techniques of plastic-aesthetic nasal surgery. 


\section{Inhaltsverzeichnis}

Zusammenfassung

Abstract

1. Einleitung

2. Qualität in der Medizin im Fokus der Rhinoplastik

2.1 Anforderung an die Qualität

2.1.1 Aus ärztlicher Sicht

2.1.1.1 Ärztliches Zentrum für Qualität in der Medizin (ÄZQ)

2.1.1.2 Arbeitsgemeinschaft der Wissenschaftlichen Medizinischen Fachgesellschaften (AWMF)

2.1.2 Aus Patientensicht

2.1.3 Aus Kostenträgersicht

2.1.3.4 Gesetzliche Krankenversicherung (GKV)

2.1.3.5 Private Krankenversicherung (PKV)

2.1.3.6 Gesetzliche Unfallversicherung (GUV)

3. Indikatoren der Qualität

3.1 Qualitätskriterien und Qualitätsindikatoren

3.1.1.1 Versorgungsaspekte

3.1.1.2 Qualitätskriterien

3.1.1.3 Qualitätsindikatoren/Referenzbereiche

3.1.1 Aus ärztlicher Sicht

3.1.2 Aus Patientensicht

3.1.3 Aus Kostenträgersicht

4. Kontrolle und Sicherung der Qualität

4.1 QM-Methoden und -Instrumente

4.1.1 Aus ärztlicher Sicht

4.1.1.1 Qualitätsziele zur Patientenorientierung aus Sicht der Ärzteschaft S174

4.1.1.2 Praktische Umsetzung der Patientenorientierung im QM

4.1.1.3 Leitlinien und Qualitätsmanagement

4.1.1.4 Aufgaben und Ziele von Leitlinien

4.1.1.5 Wirksamkeit und Qualität von Leitlinien

4.1.1.6 Nutzung von Leitlinien im Qualitätsmanagement Leitlinien-Implementierung

4.1.1.7 Umsetzung von Leitlinien in der ambulanten Versorgung

4.1.1.8 Leitlinien in der Rhinoplastik

4.1.1.9 Qualität und Patientensicherheit

4.1.1.10 CIRS medical

4.1.2 Aus Patientensicht

4.1.2.1 Patientenbefragung im Qualitätsmanagment

4.1.2.2 Patientenbeschwerden als Instrument im QM

4.1.3 Aus Kostenträgersicht
4.1.3.1 Institut für Qualität und Wirtschaftlichkeit im Gesundheitswesen (IQWiG)
S165

S165

S166

S167

S167

S167

S167

S168

S169

S170

S170

S170

S170

S170

$\mathrm{S} 170$

S171

S171

S171

S172

S172

S173

S174

S174

S174

S174
S174

S175

S175

S175

S177

S177

S177

S178

S178

S178

S178

S178

S179
4.1.3.2 Institut für Qualitätssicherung und Transparenz im Gesundheitswesen (IQTiG)

4.1.3.3 Qualität und Kosten am Beispiel der Septorhinoplastik $\quad$ S179

5. Evidenzbasierte Medizin $\quad$ S180

5.1.1 Definition und Hintergrund $\quad \$ 180$

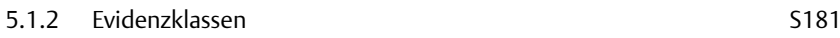

5.1.3 Evidenzbasierte Medizin und Rhinoplastik S181

6. Schlussfolgerung zum Teil $1 \quad$ S181

7. Teil 2 - Qualität von Operationsmethoden in der Rhinoplastik S182

8. Offenen vs. geschlossenen Zugang $\quad \mathrm{S} 182$

8.1.1 Qualität der äußeren bzw. ästhetischen Ergebnisse S183

8.1.2 Qualität der funktionalen bzw. endonasalen Ergebnisse \$183

8.1.3 Qualität der subjektiven Patienteneinschätzung S183

9. Rippen-vs. Ohrknorpeltranplantat S184

9.1.1 Qualität der äußeren bzw. ästhetischen Ergebnisse S184

$\begin{array}{ll}\text { 9.1.1.1 Autologer Rippenknorpel (ACC) } & \text { S184 }\end{array}$

9.1.1.2 Bestrahlter homologer Rippenknorpel (IHCC) S184

9.1.1.3 Qualität der subjektiven Patienteneinschätzung zwischen ACC und IHCC $\quad \$ 184$

9.1.1.4 Gewürfelter Knorpel in Faszie (Diced Cartilage in Fascia) $\quad$ S185

9.1.1.5 Faszienfreier loser gewürfelter Knorpel (Free Diced Cartilage) \$ \$185

9.1.1.6 Ohr- bzw. Conchaknorpel $\$$

9.1.2 Qualität der subjektiven Patienteneinschätzung zwischen Rippen- und Ohrknorpel $\quad$ S185

10. Ultraschallbasierte vs. konventionelle Osteotomie $\$ 185$

10.1.1 Qualität der äußeren bzw. ästhetischen Ergebnisse $\$ 186$

10.1.2 Qualität der funktionalen bzw. endonasalen Ergebnisse \$186

10.1.3 Qualität der subjektiven Patienteneinschätzung S186

11. Preservation vs. traditionelle Rhinoplastik $\$ 186$

11.1.1 Qualität der äußeren bzw. ästhetischen Ergebnisse \$187

11.1.2 Qualität der funktionalen bzw. endonasalen Ergebnisse \$187

11.1.3 Qualität der subjektiven Patienteneinschätzung $\quad$ S187

12. Patient Reported Outcomes (PROs) $\$ 187$

12.1.1 Qualität der äußeren bzw. ästhetischen Ergebnisse und der Lebensqualität

S188

12.1.2 Qualität der funktionellen bzw. endonasalen Ergebnisse und der Lebensqualität

12.1.3 Qualität der subjektiven Patienteneinschätzung bei PROM in der Rhinoplastik Literatur S190

\section{Einleitung}

Die Eingrenzung von Qualitätsmerkmalen im Rahmen der Effektivitätsbewertung verschiedener Therapiegebiete der HNO-Heilkunde nehmen mittlerweile breiten Raum in der Darstellung unseres
Faches ein dem sich auch der Teilbereich der plastisch-ästhetischen Rhinoplastik nicht mehr entziehen kann. Dies beschränkt sich nicht allein auf die Nutzenbewertungsbetrachtungen des gemeinsamen Bundesausschusses (G-BA), oder von Kostenträgern angestrengte 
Regulierungsinstrumentarien, sondern erstreckt sich über die Leitlinien bis hin zu dem anspruchsvollen Begriff der Evidenz. In Abgrenzung des Begriffs „Evidenz“ berührt der Qualitätsbegriff ungleich mehr, da er sich aus ärztlicher Sicht auf die Struktur der Leistungserbringung, die Güte der Ausbildung des Fachpersonals, das Gesamtpaket der Vor- und Nachbehandlung und auf den in vielen Behandlungsfeldern wichtigen Begriff der Interdisziplinarität erstreckt.

Vom „Institute of Medicine of National Academy of Sciences, USA, “ wurde 1990 folgende Definition des Qualitätsbegriffs entwickelt: „Quality of care is the degree to which health services for individuals and populations increase the likelihood of desired health outcomes and are consistent with current professional knowledge“ bzw. übersetzt: Qualität der Behandlung ist das Maß, in dem die gesundheitliche Versorgung von Individuen oder Gruppen die Wahrscheinlichkeit erhöht, daß vom Patienten erwünschte, auf die Gesundheit bezogene Ergebnisse erzielt werden und zwar in Übereinstimmung mit dem aktuellen Wissen des Berufsstandes. Die Besonderheit dieser Definition liegt für den Patienten darin, dass sie „patientenzentriert“ formuliert ist. Nicht das Ergebnis medizinischen Handelns allein steht im Mittelpunkt sondern gerade das vom Patienten wahrgenommene (idealerweise erwünschte) Ergebnis (patient reported outcome, PRO), dieses jedoch gemessen an dem fundierten Erkenntnisstand der Medizin (Leitlinien, Evidenz). Insbesondere diesen Bereich des PRO muss sich die plastisch-ästhetische Rhinoplastik stellen, da die Veränderung des Äußeren angestrebtes Ziel des Eingriffs ist.

Der Qualitätsaspekt wird sehr gerne, aber damit auch hoch inflationär und nicht klar in seiner genauen Bedeutung abgegrenzt, auf werbenden ärztlichen Hompages und Social Media Portalen, aber auch von Kostenträgern und Industrie bemüht. Dies kommt auch in einer zunehmenden Zahl von Zertifikaten und sogenannten „Qualitätslabels “ zum Ausdruck. Häufig ist jedoch aus Patientensicht unklar, wofür diese genau stehen, auf welchen Grundlagen sie beruhen und welches ihre Gültigkeitsdauer ist. Die Messung und Erfassung der medizinischen Behandlungsqualität ist allerdings aufwändig, methodisch nicht einfach und fehleranfällig sowie von subjektiven Einflüssen insbesondere im Bereich der Ästhetik überlagert.

Kaum ein Gebiet in der HNO-Heilkunde unterliegt einem schnelleren Wandel von speziellen Operationstechniken als die plastischästhetische Nasenchirurgie. Bislang gibt es Versuche, neben der intensiven Diskussion und Ergebnisbetrachtung von Operationstechniken im interdisziplinären Forum (Kongresse, Publikationen, Empfehlungen bekannter Rhinoplastiker), über eine Objektivierung der persönlichen Expertise (Mindestmengen, Vortrags-/Publikationstätigkeit usw.) gewisse Qualitätsindikatoren für Patienten und zuweisende Ärzte anzubieten. Um mögliche Täuschungen zu vermeiden ist die Werbung mit unüberprüfbaren Vorher-Nachher Bildern generell verboten worden. Register oder Qualitätskontrollmechanismen sind nicht oder unzureichend vorhanden. Die Angabe des Angebots einer Rhinoplastik auf einer Homepage lässt in der Regel offen, auf welchem Qualitätsniveau diese Leistung erbracht wird. Da es sich in diesem Gebiet um teilweise reine Selbstzahlerleistungen handelt, reagieren die Patientenströme überwiegend auf individuelle Empfehlungen. Vergleichende Studien nach Vorbild der Onkologie sind leider absolute Ausnahmen im Gebiet der ästhetischen Chirurgie und insbesondere im Bereich der Rhinoplastik.
Im ersten Teil des Referates beleuchten wir den Begriff Qualität mit dem Fokus in der Nasenchirurgie hinsichtlich seiner Anforderung, seiner Indikatoren, deren Kontrolle und Sicherung aus den drei Hauptperspektiven des Arztes, des Patienten und des Kostenträgers. Der zweite Teil wird die Qualitätsentwicklung in den einzelnen Bereichen der plastisch-ästhetischen Nasenchirurgie anhand unterschiedlicher Operationsmethoden fokussieren.

\section{Qualität in der Medizin im Fokus der Rhino- plastik}

\subsection{Anforderung an die Qualität}

\subsubsection{Aus ärztlicher Sicht}

Der Qualitätsbegriff ist ein steter Begleiter des Arztes in der Medizin und keine Erfindung der Neuzeit. Das heutige Verständnis von Qualität wurde in den ursprünglichen Definitionen unter dem Überbegriff der Ethik des ärztlichen Handelns verstanden (Eid des Hippokrates: „Meine Verordnungen werde ich treffen zu Nutz und Frommen der Kranken, nach bestem Vermögen und Urteil; ich werde sie bewahren vor Schaden und willkürlichem Unrecht"). Daran hat sich bis heute nichts verändert. Allerdings konnte sich auch der Eid des Hippokrates einer „Reformation“ im Bilde des heutigen Selbstverständnisses von Patienten nicht entziehen. So heißt es in der aktuellen erneuerten Fassung des Eids, der 2017 im Rahmen der 68. Vollversammlung der Medical World Association (WMA) in Chicago verabschiedet wurde: (The Physician's Pledge: „The health and wellbeing of my patient will be my first consideration and I will respect the autonomy and dignity of my patient“) [1].

Der Arzt befindet sich somit im Spannungsfeld zwischen seinem Anspruch, dem neuen Selbstbewußtsein der Patienten und der Interpretation von Qualität aus Kostenträgersicht, die heutzutage zunehmend mit Wirtschaftlichkeit verwechselt wird. Aus dieser Entwicklung heraus haben sich in den letzten Jahrzenten verschiedene Zentren und Institute unter Federführung verschiedener ärztlicher Organe gebildet, die den Qualitätsbegriff aus ärztlicher Sicht beleuchten, definieren und weiterentwickeln sollen.

\subsubsection{1 Ärztliches Zentrum für Qualität in der Medizin (ÄZQ)}

Das Ärztliche Zentrum für Qualität in der Medizin (ÄZQ) ist das gemeinsame Kompetenzzentrum von Bundesärztekammer (BÄK) und Kassenärztlicher Bundesvereinigung (KBV) für Qualität und Wissenstransfer im Gesundheitswesen. Es wurde 1995 als „Zentralstelle der deutschen Ärzteschaft zur Qualitätssicherung in der Medizin“ mit dem Zweck der Qualitätssicherung der ärztlichen Berufsausübung gegründet. Sie wurde im Jahre 2003 in „Ärztliches Zentrum für Qualität in der Medizin (ÄZQ)“ umbenannt. Die Aufgabe des ÄZQ besteht darin, die BÄK und KBV bei ihren Aufgaben im Bereich der Qualitätssicherung der ärztlichen Berufsausübung zu unterstützen.

Unter dem Schlagwort der Qualitätsverbesserung im Gesundheitswesen wurden ein Leitbild durch BÄK und KBV definiert. Die Grundvorraussetzung ist eine Qualitätssicherung und ein Qualitätsmanagement (QS/QM), welches bereichsübergreifend in allen Versorgungsbereichen entworfen und etabliert wird. Des Weiteren müssen Prioritäten gesetzt und der Bereich der QS/QM lösungsorientiert weiterentwickelt werden. Leitlinien und Prinzipien der 
Evidenzbasierten Medizin sollen und müssen in der Versorgung erarbeitet und die Patienten miteinbezogen werden. Versorgungsanbieter sollen angemessene personelle und organisatorische Strukturen für QS/QM schaffen, um damit eine Professionalisierung auf dem Gebiet der QS/QM in Kooperation aller Beteiligten weiter zu entwickeln [2]. Definierte Grundsätze der Arbeitsweise des ÄZQ sind die Evidenzbasierte Medizin, die Patientensicherheit, die Patientenorientierung und die Transparenz. Alles Begriffe, denen wir in der Betrachtung von Qualität im weiteren Referat wieder begegnen werden

\section{Aufgabenschwerpunkte}

Die Schwerpunkte teilen sich, wie folgt, in 4 große Blöcke auf:

- Medizinische Leitlinien (Entwicklung, Bewertung, Verbreitung, Methodik)

- Versorgungsleitlinien

- Leitlinien

- Leitlinien Clearing

- Patienteninformationen (Entwicklung, Bewertung, Verbreitung, Methodik)

- Patienteninformationsdienst

- Patientensicherheit/Fehlervermeidung in der Medizin

- Patientensicherheitonline.de

- CIRSmedical

- Qualitätsentwicklung in der Medizin

An diesen 4 Blöcken zeigt sich, dass die Schwerpunkte nicht nur die ärztliche Sicht, sondern auch den Patienten und dessen Information und Sicherheit mit einbeziehen.

Hier spiegelt sich die zuvor vom „Institute of Medicine of National Academy of Sciences, USA“, im Jahre 1990 genannte festgelegte Definition des Qualitätsbegriffs wider [3]: „....die Wahrscheinlichkeit erhöht, daß vom Patienten erwünschte, auf die Gesundheit bezogene Ergebnisse erzielt werden und zwar in Übereinstimmung mit dem aktuellen Wissen des Berufsstandes“.

Die Besonderheit dieser Definition liegt darin, dass sie „patientenzentriert“ formuliert ist. Nicht das Ergebnis medizinischen Handelns allein steht im Mittelpunkt, sondern gerade das vom Patienten wahrgenommene und idealerweise erwünschte Ergebnis.

In der Literatur wird dies als „patient reported outcome“ (PRO) definiert und muss sich an dem fundierten Erkenntnisstand der Medizin, welche sich in Leitlinien und der Evidenz ausdrückt, messen lassen. Hierzu kommen wir in Teil 2 Kapitel 12 näher zu sprechen, denn PROs spielen in der ästhetischen Chirurgie und insbesondere der Rhinoplastik eine wesentliche Rolle.

\subsubsection{Arbeitsgemeinschaft der Wissenschaftlichen Medizini- schen Fachgesellschaften (AWMF)}

Eine der ersten Fachzusammenschlüsse, um die Qualität der ärztlichen Tätigkeit zu benennen und zu sichern, wurde 1962 durch die Gründung der Arbeitsgemeinschaft der Wissenschaftlichen Medizinischen Fachgesellschaften (AWMF) initiiert. Damals trafen sich auf Anregung der Deutschen Gesellschaft für Chirurgie Vertreter von 16 wissenschaftlich-medizinischen Fachgesellschaften, um v. a. die fachärztliche Weiterbildung und die Einführung einer Facharztprüfung zu implementieren. Die AWMF hat sich in den darauffolgenden
30 Jahren in allen Fachgesellschaften mit einer ganzen Reihe von Problemstellungen befasst, wie z. B. die Weiterbildungsordnung für Ärzte (1962, kontinuierlich seit 1987), die Approbationsordnung für Ärzte (seit 1970), die Qualitätssicherung ärztlicher Berufsausübung (seit 1979 bzw. seit 1993 kontinuierlich) usw. Sie sorgte somit seither für eine Qualitätsverbesserung der Ausbildung durch Festlegung von zu erfüllenden Standards.

Heute sind in der AWMF über 150 wissenschaftlich-medizinische Fachgesellschaften vertreten, die sich im Rahmen der AWMF mit der gesamten Palette wissenschafts- und forschungspolitischer Fragen im Bereich der Medizin beschäftigen und mit anderen Organisationen, wie z. B. BÄK, Medizinischer Fakultätentag, Verband der Universitätsklinika und den Einrichtungen der Wissenschaftsförderung (z. B. Deutscher Forschungsgemeinschaft) ein wichtiger Pfeiler im Rahmen der gesamten deutschen Medizinversorgung sind. Diese Arbeit mündete dahingehend, dass der Sachverständigenrat für die „Konzertierte Aktion im Gesundheitswesen“ schon 1995 die AWMF beauftragte die Entwicklung von Standards, Richtlinien, Leitlinien und Empfehlungen der Wissenschaftlichen Medizinischen Fachgesellschaften voranzutreiben und zu koordinieren, um eine weitere Qualitätsbestimmung zu erreichen > Abb. 1.

\section{Leitlinien}

Prinzipiell sind drei Programme im Bereich der Leitlinien zu unterscheiden. Die Nationalen Versorgungsleitlinien, die allesamt dem höchsten Status S3 entsprechen. Dem „Leitlinienprogramm Onkologie“ in Zusammenarbeit mit der Deutschen Krebsgesellschaft und der Deutschen Krebshilfe, die ebenfalls alle die höchste evidenzbasierte Leitlinienstufe S3 besitzen und als drittes die Leitlinien anderer Fachgesellschaften zur Verifizierung von diagnostischen und therapeutischen Vorgehensweisen, die allerdings alle Stufen von S1 bis S3 repräsentieren. Unabhängig von der Stufe der Leitlinie ist sie heute ein fester Pfeiler im QM, worauf später im Referat noch einzugehen ist.

Den Begriff Leitlinie definiert die AWMF wie folgt:

„Die Leitlinien der Wissenschaftlichen Medizinischen Fachgesellschaften sind systematisch entwickelte Hilfen für Ärzte zur Entschei-

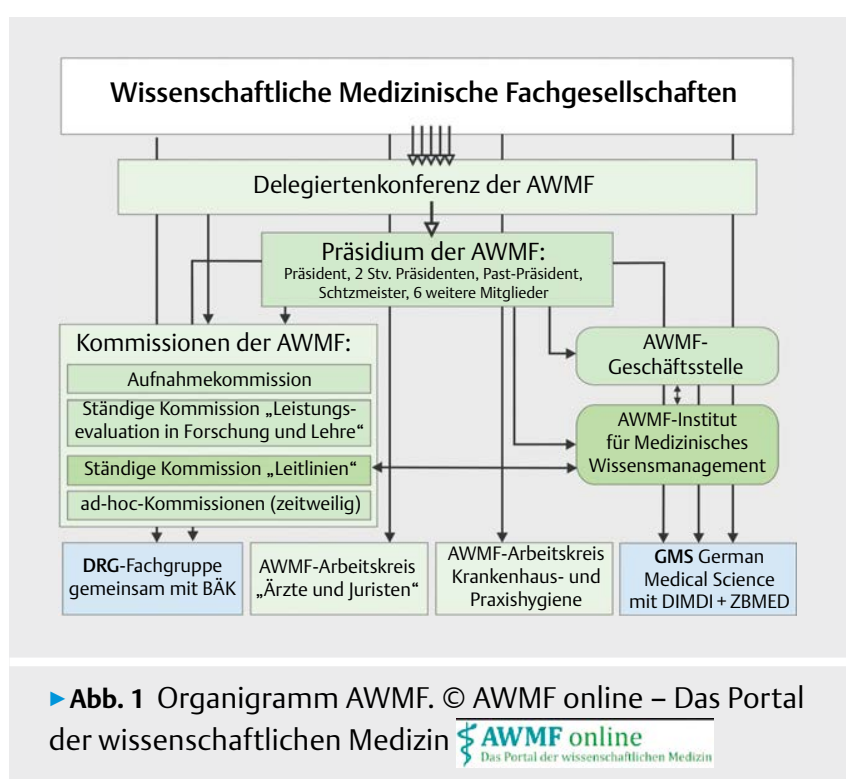


dungsfindung in spezifischen Situationen. Sie beruhen auf aktuellen wissenschaftlichen Erkenntnissen und in der Praxis bewährten Verfahren und sorgen für mehr Sicherheit in der Medizin, sollen aber auch ökonomische Aspekte berücksichtigen. Die Leitlinien sind für Ärzte rechtlich nicht bindend und haben daher weder haftungsbegründende noch haftungsbefreiende Wirkung“.

Mittlerweile sind alleine unter der Federführung der Deutschen Gesellschaft für Hals-Nasen-Ohren-Heilkunde, Kopf- und Hals-Chirurgie (DGHNOKHC) 20 Leitlinien unterschiedlicher Stufen erarbeitet worden. Des Weiteren war die DGHNOKHC bei 45 weiteren Leitlinien in deren Ausarbeitung und Definierung beteiligt.

Unter den 20 durch die Federführung der DGHNOKHC erstellten Leitlinien sind alle Klassifikationsstufen vertreten von S1 (z. B.: Ösophagoskopie, Hörsturz), über S2k (z. B.: Rhinochirurgie und Rhinosinusitis), S2e (z. B.: Schlafapnoe) bis zu S3 (z. B.: Larynxkarzinom, Tinnitus). Die Leitlinie für die Rhinochirurgie steht auf der Stufe $\$ 2 \mathrm{k}$, ist somit konsensusbasiert und wird im Kapitel 4.1.1.3 weiter besprochen werden. Sie ist ein wesentlicher Schritt hin zu mehr Qualität in der plastisch-ästhetischen Nasenchirurgie.

\subsubsection{Aus Patientensicht}

Der Bereich der Qualität der gesundheitlichen Versorgung ist für Patienten oftmals eine Frage von existentieller Bedeutung. Betrachtet man nationale und internationale Studien zu den Erwartungen, die Patientin an die Qualität einer medizinischen Versorgung richten, lassen sich zusammenfassend 7 große Themenbereiche formulieren, wobei der letzte für die Rhinoplastik eher eine untergeordnete Bedeutung hat. [4-5]

- Wiederherstellung von Gesundheit und körperlichem Wohlbefinden

- Respekt vor der Person des Patienten, Respekt vor dessen subjektiven Werten und Vorstellungen

- Ausreichende und verständliche Informationen

- Emotionale Unterstützung und Empathie

- Fachgerechtigkeit und Kompetenz der medizinischen Behandlung aber auch der Sonstigen, z. B. pflegerischen Betreuung

- Einbeziehung von Partner, Familie und Freunden

- Kontinuität der Versorgung, verstanden als personelle Kontinuität innerhalb der medizinischen Einrichtung und als Kontinuität zwischen den einzelnen Versorgungsbereichen, etwa als Hilfestellung bei Entlassung aus den Krankenhäusern in die häusliche Umgebung

Der Bedarf an Informationen über die Qualität der gesundheitlichen Versorgung in den genannten Dimensionen ist für Patienten außerordentlich, wie nicht zuletzt schon die Arbeiten Anfang der 2000er Jahre deutlich machen [6].

Die Patienten wollen zunehmend genauer wissen in welcher Einrichtung und durch welchen Arzt sie qualitativ hochwertig versorgt werden. Dieser Wunsch spiegelt ein neues Selbstverständnis und Selbstbewußtsein der Patienten wider. Sie sehen sich nicht nur als Nutzer der ärztlichen Dienstleistung, sondern möchten auch als Partner auf Augenhöhe durch den Arzt verstanden werden. Dies spielt im Bereich der Rhinoplastik eine wesentliche Rolle, da diese Patienten als äußerst herausfordernd empfunden werden.
Anscheinend gibt es darüberhinaus offenbar eine wachsende Verunsicherung, in Bezug auf die Qualität der medizinischen Versorgung. Zwei Drittel (!) der Bevölkerung haben schon vor 18 Jahren konstatiert, daß sich die Situation des Patienten im Gesundheitswesen im Rahmen der letzten Jahre eher verschlechtert habe [7].

Im Vergleich mit anderen Industrienationen ergab sich 2015 ein heterogenes Bild: In manchen Bereichen nimmt Deutschland einen Spitzenplatz ein (z. B. hausärztliche Versorgung), allerdings ist jeder fünfte Befragte der Ansicht, dass das deutsche Gesundheitssystem komplett reformiert werden sollte [8].

Die Möglichkeiten sich über die Strukturprozesse und Ergebnisqualität eines Arztes und/oder eines Krankenhauses transparent zu informieren, werden durch Patienten immer mehr eingefordert, auch bedingt durch die allgemein zugängliche Information hierüber im Internet und den sozialen Medien. Patienten erkundigen sich über die ärztliche Versorgung und Qualität darüberhinaus entweder im Bekannten- und Freundeskreis oder über niedergelassene Ärzte. Diese Erfahrungen und Zufriedenheitsäußerungen, die in sozialen Netzwerken und im Internet geäußert werden, haben einen zunehmend hohen Stellenwert, obwohl sie größtenteils auf ihre Echtheit und Authentizität nicht zu überprüfen sind.

In einer Umfrage gaben ein Drittel der Patienten an, die medizinische Einrichtung auf Empfehlung des Arztes ausgewählt zu haben [9]. 20\% verließen sich auf die Empfehlung von Angehörigen und Bekannten, ca. $18 \%$ hatten schon frühere Aufenthalte in dieser Klinik, 17\% geben den allgemeinen Ruf des Hauses als ihre Wahl an für $12 \%$ war es die räumliche Nähe und $6 \%$ haben sich über das Internet für das Krankenhaus entsprechend entschieden. Auch Selbsthilfegruppen, die für ihre Mitglieder und weitere Interessengruppen als wichtige Informationsbörse dienen, gehören hierzu.

Der Arzt, der einen Patienten zu einer Kollegin oder einem Kollegen überweist, spricht direkt bzw. indirekt eine Empfehlung aus, an der sich die Patienten entsprechend orientieren. Die Empfehlung des Arztes kommt größtenteils aus der eigenen Erfahrung in der Zusammenarbeit mit bestimmten Kollegen, Abteilungen, Stationen oder Informationen aus dem professionellen Netzwerk bzw. die Rückmeldung der eigenen Patienten.

Im Kontext von Qualitätsmanagement und der Frage nach einer evidenzbasierten Patientenversorgung belegen Untersuchungen in verschiedenen medizinischen Bereichen, dass eine ausschließliche Konzentration der Behandlungsmethoden und -verfahren auf körperliche Symptomatik und Behandlungseffekte nur unzureichende Wirksamkeiten aufweisen [10]. Aus diesem Grund werden bei der Messung der Behandlungseffektivität zunehmend auch psychoemotionale Merkmale der Arzt/Patienten Beziehung, die Kommunikation und eine partnerschaftliche Entscheidungsfindung berücksichtigt (s. o.) $[11,12]$. Das Wissen über die persönlichen Lebenswelten und Wertesysteme der einzelnen Patienten, das Erleben ihrer Erkrankung sowie ihre Bewältigungsstrategien müssen erst kommuniziert werden, um eine adäquate Behandlungsmethodik auszuwählen, die den jeweiligen Bedürfnissen entspricht [13]. Der Aufbau einer stabilen Vertrauensbasis, welche dem Patienten Verständnis für die individuellen Belange und Bedürfnisse signalisiert, ist für die Qualität einer Therapie aus Patientensicht von zentraler Bedeutung [14]. Das Verständnis und das Eingehen auf die Bedürfnislage, Erklärungen, wirksame Therapien und fachliche Informationen sind Indizien für die Fachkompetenz des Arz- 
tes aus der selbstbewußten Patientensicht [15]. Diesem Trend können sich auch die chirurgischen Fächer nicht entziehen. Somit sind die Erfolgsparameter nicht ausschließlich in der Festlegung evidenzbasierter Standards zu verorten, sondern vielmehr in der subjektiven Bewertung des Therapiegeschehens durch den Patienten, das ein weiteres wichtiges Qualitätskriterium darstellt [16]. Dieser Komplex der Kommunikation, Interaktion, Verständigung, Bewertung usw. ist für die plastisch-ästhetische Nasenchirurgie von größter Bedeutung, weil es sich um einen äußerlichen formverändernden Eingriff handelt, der auch noch mitten im Gesicht stattfindet. Die Erwartungshaltung des Patienten muss vom Arzt adäquat eingeschätzt werden.

Die oben schon erwähnten subjektiven Bewertungen (PROs) spielen im ästhetischen Bereich eine ganz wesentliche Rolle, denn Schönheit liegt im Auge des Betrachters und ist zum Teil auch abhängig vom Zeitgeist und der gesellschaftlichen Gesamtsituation. Des Weiteren liefert die Bewertung von den genannten Kriterien Erkenntnisse über den tatsächlich von den Patienten wahrgenommenen Qualitätsstandard der erhaltenen Leistung und der subjektiv empfundenen Verbesserung des Gesundheitszustands. Die gleichzeitige Erfassung der Patientenbedürfnisse stellt ein konsequent patientenorientiertes und effektives Instrument zur Qualitätssicherung dar, um die Prozess- und Ergebnisqualität aus der Nutzerperspektive ganzheitlich zu erfassen. Die sich daraus ergebende Weiterentwicklung in generische (allgemeine) und krankheitsspezifische subjektive Messinstrumente, sogenannte PROMs (Patient related outcome measurements), ist im vollen Gange und wird später, insbesondere unter Berücksichtigung der Rhinoplastik, im Kapitel 12 zu beleuchten sein.

\subsubsection{Aus Kostenträgersicht}

Prinzipiell muss man bei den Kostenträgern die Gesetzliche Krankenversicherung bzw. die Private Krankenversicherung sowie die Gesetzliche Unfallversicherung und Berufsgenossenschaften trennen. Sie sind in unterschiedlichen Sozialgesetzbüchern (SGB) beschrieben und abgegrenzt. Ihre Aufgaben sind verschieden definiert und drücken sich auch in den verschiedenen Handlungsmaximen aus.

\subsubsection{Gesetzliche Krankenversicherung (GKV)}

Ein wesentlicher Pfeiler für die Gesetzliche Krankenversicherung liegt in der Wirtschaftlichkeit, wie im ersten Kapitel des SGB V nachzulesen ist. So heißt es im SGB V unter dem Gesetzeskapitel 2, § 3 , Satz 1: „Die Krankenkassen stellen den Versicherten die im Dritten Kapitel genannten Leistungen unter Beachtung des Wirtschaftlichkeitsgebots (\$ 12) zur Verfügung“. Erst danach wird auf die Qualität Bezug genommen: „Behandlungsmethoden, Arznei- und Heilmittel der besonderen Therapierichtungen sind nicht ausgeschlossen. Qualität und Wirksamkeit der Leistungen haben dem allgemein anerkannten Stand der medizinischen Erkenntnisse zu entsprechen und den medizinischen Fortschritt zu berücksichtigen“.

Die Leistungserbringung und das Wirtschaftlichkeitsgebot sind im §12 (1) wie folgt definiert: „Die Leistungen müssen ausreichend, zweckmäßig und wirtschaftlich sein; sie dürfen das Maß des Notwendigen nicht überschreiten. Leistungen, die nicht notwendig oder unwirtschaftlich sind, können Versicherte nicht beanspruchen, dürfen die
Leistungserbringer nicht bewirken und die Krankenkassen nicht bewilligen“.

Damit zeigt sich schon das Dilemma unter dem die GKV steht, denn all ihre Leistungen sind unter dem Gebot der Wirtschaftlichkeit zu sehen. Die Fehlinterpretation von Wirtschaftlichkeit ist gleich Qualitätsmerkmal wird noch später zu prüfen sein.

\subsubsection{Private Krankenversicherung (PKV)}

Die private Krankenversicherung ist im Gegensatz hierzu nicht Kraft Gesetz in den Sozialgesetzbüchern definiert, sondern das Versicherungsverhältnis kommt durch einen privatrechtlichen Vertrag zustande. Die Grundlagen sind in dem allgemein gültigen Versicherungsvertrag- und Versicherungsaufsichtsgesetz (VVG und VAG) geregelt.

Für die private Krankenversicherung ist der Versicherungsfall die „medizinisch notwendige Heilbehandlung“. Ein Wirtschaftlichkeitsgebot existiert in der privaten Krankenversicherung in dieser Form explizit nicht. Es ist eher der jeweils einzelne Vertrag zwischen den Patienten und den Versicherungsunternehmen, der über die Intensität und das Ausmaß der getragenen Behandlungskosten entscheidet.

\subsubsection{Gesetzliche Unfallversicherung (GUV)}

Die Aufgabe der gesetzlichen Unfallversicherung und der Berufsgenossenschaften ist hingegen diametral anders definiert. Im Gegensatz zur gesetzlichen Krankenversicherung soll sie das Maximum der geeigneten Mittel einsetzen. Ihre Aufgaben und Tätigkeiten sind gesetzlich im Sozialgesetzbuch VII (SGB VII) geregelt. In ihrem Fokus steht nicht die allgemeine Gesundheitsversorgung, sondern die Prävention, Rehabilitation und Entschädigung ihrer Versicherten (SGB VII, §1).

Dementsprechend setzt sie in ihrem Selbstverständnis eine hohe Qualität voraus, um ihre Versicherten wieder schnell in Arbeit zu bringen. Im hierfür zuständigen Sozialgesetzbuch VII steht unter Kapitel 2, Abschnitt $1 \S 1$ (2): „Aufgabe der Unfallversicherung ist es, nach Maßgabe der Vorschriften dieses Buches nach Eintritt von Arbeitsunfällen oder Berufskrankheiten die Gesundheit und die Leistungsfähigkeit der Versicherten mit allen geeigneten Mitteln wiederherzustellen und sie oder ihre Hinterbliebenen durch Geldleistungen zu entschädigen“. Die Wirtschaftlichkeit ist hier in keinem Kapitel ausdrücklich erwähnt. Die Qualität ist zwar ebenfalls nicht explizit genannt, ergibt sich aber aus den Spezialeinrichtungen der GUV und Berufsgenossenschaften, wie z. B. den BG-Kliniken, die in ihrem jeweiligen Bereich sowohl in der Akutversorgung als auch der Rehabilitationsbehandlung hochspezialisiert auf die einzelnen Verletzungsmuster sind.

\section{Indikatoren der Qualität}

\subsection{Qualitätskriterien und Qualitätsindikatoren}

Grundlage aller Untersuchungen zur Qualität erbrachter Leistungen ist die Dokumentation und Analyse von Qualitätsindikatoren. Sie zielen auf die Beurteilung der Frage, welchen Qualitätsgrad eine erbrachte Leistung erreicht hat (Ziel erreicht/zum Teil erreicht/nicht erreicht?). Qualitätsindikatoren versuchen das zunächst nicht messbare Konstrukt „Qualität der medizinischen Versorgung“ 
greifbarer zu machen, indem für besonders wichtige Versorgungsaspekte die Qualität bei einzelnen Qualitätskriterien überprüft wird. (Beispiele: Labor, Röntgen usw.).

\subsubsection{Versorgungsaspekte}

Als Versorgungsaspekte werden Strukturen, Prozesse und Ergebnisse der medizinischen Versorgung bezeichnet, die für die Qualität der medizinischen Behandlung eine besonders hohe Relevanz haben und deshalb im Rahmen des QM beurteilt werden sollten. Für die Auswahl von Versorgungsaspekten, die in das medizinische Qualitätsmanagement einbezogen werden sollten, gibt es eine Reihe von Vorschlägen.

Demnach sollten gerade solche Versorgungsaspekte einer Bewertung zugeführt werden, die auf der Grundlage von Belegen

- mit hoher Frequenz durchgeführt werden, ein hohes Risiko für die Patienten beinhalten oder oft mit Problemen einhergehen;

- möglicherweise mit „Über-, Unter- oder Fehlversorgung“ verbunden sind;

- einer hohen Versorgungsvariabilität unterliegen, deren Versorgung sich kürzlich stark verändert hat, deren finanzielle Bedeutung hoch ist oder bei denen praktische Erwägungen positiv beschieden werden, wie die grundsätzliche Messbarkeit und Veränderbarkeit sowie eine Akzeptanz durch die Gruppe Betroffener;

- die Möglichkeit bieten, die Versorgung und das Gesundheitsergebnis tatsächlich zu verbessern, bei denen ein großes Verbraucherinteresse besteht oder mit denen die Entscheidungsfindung (für oder gegen einen bestimmten Leistungsanbieter) eventuell verbessert werden kann;

- als Indikatoren für umfassende Versorgungsprobleme gelten

Bis auf den letzten genannten Punkt trifft dies auf den Bereich der inneren und äußeren Naseneingriffe zu. Sie werden in einer hohen Zahl durchgeführt und müssen sich immer wieder der Diskussion einer adäquaten Therapie stellen (Stichwort Septumplastik). Darüberhinaus besteht ein hohes Patienteninteresse, da Atmung und Riechen essentiell für die Lebensqualität sind und dies aufgrund der Auswirkung einer Dynamik unterworfen ist, die auch messbar ist. Der ästhetische Gesichtspunkt kommt hierbei noch dazu, da er primär nicht zum Versorgungsaspekt gehört.

\subsubsection{Qualitätskriterien}

Qualitätskriterien für die Bewertung der Leistungserbringung sind solche Eigenschaften, deren Erfüllung typischerweise bei einer qualitativ hochwertigen medizinischen Versorgung erwartet werden.

Eine Liste solcher Kriterien wurde bereits 1998 von der Joint Commission on Accreditation of Healthcare Organizations (JCAHO) veröffentlicht [17]. Diese Kriterien sind bis heute aktuell, international gebräuchlich und im Folgenden aufgeführt:

- Zugänglichkeit der Versorgung

- Angemessenheit der Versorgung

- Stetigkeit/Koordination der Versorgung

- Wirksamkeit unter Idealbedingungen (Efficacy of care)

- Wirksamkeit in der Versorgungspraxis (Effectiveness of care)

- Wirtschaftlichkeit der Versorgung (Efficiency of care)

- Patientenorientierung der Versorgung
- Sicherheit der Versorgungsumgebung

- Rechtzeitigkeit der Versorgung

Im WHO „World Health Report 2000“ wird auf solche Kriterien Bezug genommen und davor gewarnt, diese eher instrumentellen Qualitätskriterien zur Gesamtbeurteilung von Gesundheitssystemen einzusetzen. Die WHO nennt für eine solche Gesamtbewertung drei Kernziele guter Gesundheitsversorgung: Gesundheit, faire Finanzierung und Patientenorientierung [18].

Qualitätskriterien für einzelne Versorgungsaspekte (z. B.: Rhinoplastik) können bspw. im Rahmen des einrichtungsinternen Qualitätsmanagements für die Definition von spezifischen Qualitätszielen genutzt werden.

Beispiele für Qualitätskriterien:

- Rechtzeitigkeit der Diagnostik bei Patienten

- Information des Patienten über seine Befundung, vorgesehene Behandlung, seine Rechte und Pflichten

- Aus-, Weiter-, Fortbildung des Praxisteams

- Führung von Diagnosen-/Behandlungsstatistiken

- angemessene Anwendung von diagnostischen und therapeutischen Maßnahmen

- Wartezeiten bei Aufnahme/vor Arztkontakt/Behandlung/in Klinik und Praxis

\subsubsection{Qualitätsindikatoren/Referenzbereiche}

Qualitätsindikatoren sind Maße, deren Ausprägung eine Unterscheidung zwischen guter und schlechter Qualität von Strukturen, Prozessen und/oder Ergebnissen der Versorgung ermöglichen sollen. Sie sind Hilfsgrößen, die die Qualität einer Einheit durch Zahlen bzw. Zahlenverhältnisse indirekt abbilden. Man könnte sie auch als auf Qualität bezogene Kennzahlen bezeichnen.

Die Qualität der Behandlung ist ein komplexes Phänomen, das in der Regel nur durch mehrere Indikatoren abgebildet werden kann. Anhand einzelner Qualitätsindikatoren kann daher nicht auf die Gesamtqualität der Patientenbetreuung, eines Leistungserbringers oder einer Einrichtung geschlossen werden. Stattdessen beleuchten einzelne Indikatoren immer nur Teilaspekte der Qualität. Es ist daher sinnvoll, Zusammenstellungen mehrerer Indikatoren zur Beurteilung einer Behandlung bzw. Qualitätskriteriums in Form von Indikatorenprofilen vorzunehmen.

Indikatoren sind unter anderem dazu geeignet, die Qualität der Patientenbetreuung, der operativen Versorgung und der patientenseitigen Behandlungsergebnisse zu beeinflussen. Darüber hinaus dienen Qualitätsindikatoren dazu, den Zielerreichungsgrad der medizinischen Behandlung selber anhand der am Patienten ablesbaren Ergebnisqualität zu bewerten (Evaluationsfunktion).

Die Bewertung erfolgt mithilfe vorab definierter Werte für „gute Qualität“, den so genannten Referenzbereichen. Der Referenzbereich ist dasjenige Intervall, innerhalb dessen die Ausprägung eines Qualitätsindikators als „gut oder unauffällig“ definiert wird. Die Bewertung einer guten Qualität ist in der Rhinoplastik sehr schwierig, da den objektiven Kriterien Atmung und Riechen das subjektive Empfinden der Ästhetik gegenübersteht. Schönheit liegt bekanntermaßen im Auge des Betrachters und differiert auch häufig zwischen Operateur und Patient.

Für einen Qualitätsindikator sind - in Abhängigkeit bestimmter Einflussfaktoren - unterschiedliche Referenzbereiche denkbar. 
Unter den Einflussfaktoren sind vor allem patientenbezogene (z. B. Ausmaß der Veränderung, Atmung, Riechen) von operateursbezogenen (z. B. Erfahrung, Op-Zahlen, Spezialisierung) zu unterscheiden. Insbesondere die Indikatoren zur Erhebung der Ergebnisqualität reagieren sensibel auf die vorgenannten Einflussfaktoren. Die Diskriminationsfähigkeit von Qualitätsindikatoren mit ihren zugehörigen Referenzbereichen wird wesentlich durch deren Sensitivität und Spezifität beeinflusst. Bei einem optimal gewählten Referenzbereich werden nahezu alle Qualitätsprobleme erkannt (hohe Sensitivität), ohne dabei zu viel „falschen Alarm“ zu produzieren (hohe Spezifität). Dabei sind die positiv und negativ vorhersagbaren Werte von der Frequenz der Qualitätsprobleme abhängig.

Die Realisierung von Programmen zur Entwicklung und Anwendung von Qualitätsindikatoren wird - ebenso wie die von Leitlinien - maßgeblich von deren Qualität, Praktikabilität und Finanzierbarkeit beeinflusst. Aus diesem Grund sind auch für Qualitätsindikatoren „Qualitätskriterien“ beschrieben worden, die in Teilen den Qualitätskriterien für Leitlinien entsprechen (siehe Kapitel 4.1.1) $[19,20]$.

\subsubsection{Aus ärztlicher Sicht}

Qualitätskriterien, die aus ärztlicher Sicht für die Versorgung der Patienten eine wesentliche Rolle spielen, speisen sich aus dem, was Ärzte im Rahmen ihrer Ausbildung kennengelernt und erlernt haben, sowie die Bereiche, die sie aus dem Austausch im Kollegenkreis oder durch Kongresse, Symposien bzw. Informationen seitens der Fachgesellschaften erhalten haben. Hierzu zählt insbesondere die Art, Intensität und Dauer der Facharztausbildung der Kollegen, die eng mit der jeweiligen Ausbildungs- und Lehrklinik oder Schwerpunktklinik verknüpft sind. Darüberhinaus kommen auch Publikationen, Vorträge oder eine Lehrtätigkeit als wesentliche Pfeiler von Qualitätsindikatoren aus ärztlicher Sicht in Betracht. Allerdings ist insbesondere im Bereich der Rhinoplastik mittlerweile eine inflationäre Anzahl an OP-Kursen usw. weltweit im Angebot. Man kann inzwischen fast 2-mal pro Woche an unterschiedlichen Veranstaltungen zu diesem Thema teilnehmen. Die Qualität solcher Kurse unterliegt keiner Qualitätskontrolle oder Mindeststandards und kann von jedem Arzt angeboten werden. Dies ist aber kein singuläres Problem der Nasenchirurgie, sondern leider für alle Bereiche der Medizin zu registrieren. Aus all diesen einzelnen Parametern und Erfahrungswerten generiert ein Arzt seine persönlichen Qualitätsindikatoren bzw. Qualitätskriterien, um für sich eine Bewertung anderer Kollegen zu erreichen, die man in einem Satz zusammenfassen könnte: „Wo würde ich selbst hingehen? Zu wem habe ich vertrauen?"

Darüberhinaus ist der sogenannte Facharztstandard, der zusätzlich auf zwei Säulen ruht, von entscheidender Bedeutung in der Qualitätsanalyse.

Eine Säule steht für die Menge der versorgten Krankheitsbilder aus einem Fachbereich (z. B.: HNO) und somit die Erfahrung des Arztes in diesem Fach. Die andere Säule steht entweder für die Variation des individuellen Spektrums (z. B.: allgemeine HNO) oder die diagnostische und therapeutische Spezialisierung des Arztes und seine Expertise für diese Disziplin (z. B.: Nasenchirurgie, Ohrchirurgie, Allergologie, Tumoren usw.). Diese Bereiche münden häufig in den Daten einer geringeren Nebenwirkungs- und Komplikationsrate und einer hohen Ergebnisqualität, die seitens der Patien- ten gegenüber dem über- oder zuweisenden Arzt kommuniziert und auch durch sie selbst und die Kostenträger zunehmend eingefordert werden.

Insbesondere in der Rhinochirurgie stehen die Ergebnisse im Vordergrund, die augenscheinlich optisch ein gutes postoperatives Ergebnis zeigen, was nichts über das Innere der Nase aussagt. Erst der HNO-Facharzt kann durch eine endoskopische Befundung dies auch für das Innere der Nase bestätigen. Es bleibt aber immer zu beachten, dass die objektiv erhobenen Ergebnisse nicht mit der subjektiven Empfindung des Patienten korrelieren müssen, insbesondere im Bereich Atmung, Riechen und Optik. Die Erfahrung eines Operateurs spiegelt sich ebenfalls in den Revisions- und Rekonstruktionsfällen wider, die er operiert, denn in der Rhinochirurgie sind hierfür eine ausdrückliche Erfahrung mit hoher Fallzahl und Expertise besonders wichtig.

\subsubsection{Aus Patientensicht}

Indikatoren für die Arztwahl sind äußerst unterschiedlich und hängen sowohl vom Krankheitsbild als auch dem Fachbereich ab. Die Auswahl eines Gynäkologen setzt sich aus anderen Prioritäten als die eines Tumorchirurgen zusammen. Nichtsdestotrotz haben auch Patienten Indikatoren, die ihnen helfen die Qualität einzuschätzen. Qualitätsbewusste Patienten bewerten nicht nur die erhaltene medizinische, therapeutische, pflegerische Versorgung und das Ergebnis der Behandlung, sondern auch die Informationsvermittlung, die Kommunikation mit den Leistungserbringern, den Eindruck vom Personal (Freundlichkeit, Zuverlässigkeit, Kompetenz, Pünktlichkeit, Wartezeit), den Umgang mit allen Mitarbeitern der Praxis oder der Klinik, den Zugang zur und die Organisation in der Praxis sowie die Unterstützungsangebote (s. o.) [21].

Hierbei zeichnen sich schon 2 wesentliche Bereiche ab, die man als „harte“ und „weiche“ Faktoren beschreiben könnte. Unter harten Faktoren bezeichnen wir die, welche durch entsprechende Fakten objektiv oder zum Teil objektiv für den Patienten nachzuvollziehen sind.

Harte Fakten:

- Objektive Kriterien:

- Berufserfahrung

- Ausbildungsgrad

- Facharzttitel

- Praxisausstattung

- Personal

- Hygiene

- Teilweise objektive Kriterien:

- persönliche Empfehlung

- Bewertungsportale und Foren

- Onlineauftritt

- Siegel

- Praxiseinrichtung

- Mitglied in einer Fachgesellschaft

Demgegenüber stehen so genannte weiche Faktoren, die eher dem subjektiven Empfinden des Patienten entsprechen.

- Weiche Faktoren:

- Persönlichkeit des Arztes

- Arzt hörtzu

- Arzt lässt Fragen zu 
- Arzt erklärt

- Arzt respektiert mich und meine Probleme als Patient

- Arzt ist an meinem gesundheitlichen Problem interessiert

- Arzt nimmt sich Zeit

- Arzt-Patientenvertrauen

Aus diesem Blickwinkel heraus haben die BÄK, die KBV und das ÄZQ im Jahre 2015 eine allgemeine Checkliste für Patienten herausgegeben, die diesen Bereich beleuchten und bündeln soll [22].

Im Vorwort dieses Werkes heisst es:

„Sie müssen zum Arzt und wissen nicht so recht wohin. Ihre Ärztin oder Ihr Arzt soll fachlich kompetent und vertrauenswürdig sein. Mit Ihrem Anliegen möchten Sie ernst genommen werden und sich außerdem vom Praxisteam gut betreut fühlen. Es fällt jedoch schwer einzuschätzen, ob eine Arztpraxis diese Erwartungen auch erfüllen kann. Aus diesem Grund wurde die Checkliste „Woran erkennt man eine gute Arztpraxis?“ entwickelt. Diese Broschüre kann Ihnen dabei helfen, die für Sie passende Praxis zu finden. Hier können Sie lesen, was Sie von einer guten Arztpraxis erwarten dürfen. Ob eine Ärztin oder ein Arzt tatsächlich „richtig“ für Sie ist, dafür ist letztlich die vertrauensvolle Beziehung entscheidend. Sie entsteht nicht durch Checklisten, sondern durch das respektvolle und offene Miteinander“...

Als Hilfestellung sind unter anderem folgende Punkte benannt:

1. Werde ich in der Praxis freundlich und respektvoll behandelt?

2. Nimmt meine Ärztin/mein Arzt mich und mein Anliegen ernst?

3. Werden in der Praxis meine Persönlichkeit und meine Intimsphäre respektiert?

4. Erhalte ich eine verständliche und neutrale Aufklärung, Information und Beratung?

5. Bekomme ich Hinweise auf weiterführende verlässliche Informationsquellen und Beratungsangebote?

6. Bezieht meine Ärztin/mein Arzt mich und meine Wünsche in alle Entscheidungen ein?

7. Akzeptiert meine Ärztin/mein Arzt, dass ich im Zweifelsfall eine zweite Meinung einholen möchte?

8. Kann ich erkennen, ob meine Ärztin/mein Arzt und das Mitarbeiterteam an Fortbildungsveranstaltungen und Qualitätsprogrammen teilnehmen?

9. Wird in der Praxis auf möglichst große Sicherheit bei meiner Behandlung geachtet?

10. Erhalte ich ohne Probleme Zugang zu meinen Patientenunterlagen?

11. Kooperiert die Praxis mit anderen Ärztinnen/Ärzten?

Die Problematik besteht darin, dass der Patient in keiner Weise darauf hingeweisen wird, welche Punkte unter objektiven oder subjektiven Kriterien zu subsumieren sind und wie er diese einzuordnen hat. Die Liste erinnert eher an eine subjektive allgemeine Einschätzung. Über die Erfahrung, das Können, die Spezialisierung, die Ausbildung oder ähnliches geben diese Checklisten leider überhaupt keine Auskunft.

Für die plastisch-ästhetische Nasenchirurgie sind andere „Qualitätsindikatoren“ für Patienten wichtig, die sich natürlich aus objektiven aber auch sehr häufig sehr subjektiven Kriterien speisen.
Die Liste erhebt keinen Anspruch auf Vollständigkeit allerdings spielen folgende Kriterien bei Patienten, wie häufig im ästhetischen Bereich, eine Rolle:

- Was für einen Facharzt hat der Operateur?

- Wo hat er die Rhinoplastik gelernt?

- Ist er Mitglied in einer Fachgesellschaft?

- Wie häufig macht er die OP?

- Ist er spezialisiert auf Nasenchirurgie?

- Ist er spezialisiert auf Revisionschirurgie?

- Was kostet die OP?

- Was sagen andere über den Chirurgen?

- Wie gut steht er in Bewertungsportalen oder Foren da?

- Wie aufschlussreich ist die Homepage?

- Wie ist der Auftritt in den sozialen Netzwerken?

- Ist der Arzt sympathisch?

- Usw.

Es entsteht somit ein Konglomerat an „Fakten“, die den Patienten in seiner Arztwahl bewußt und unbewußt beeinflussen und die subjektiv als Qualitätsindikatoren betrachtet werden. Insbesondere die emotionalen Anteile, die durch Aussagen anderer bestärkt werden, egal ob verifizierbar oder nicht, lassen jegliches objektive Kriterium, unabhängig von der Eingriffsart, verschwinden.

\subsubsection{Aus Kostenträgersicht}

Aus Kostenträgersicht steht seit der Wiedervereinigung die Wirtschaftlichkeit im Mittelpunkt. Arbeitet ein Leistungserbringer effizient und wirtschaftlich, so sollte die Qualität dementsprechend sein. Dies war der Ansatz, den die Kostenträger seit Beginn der 1990er Jahre propagierten. Deren Fokus wurde rein auf die Wirtschaftlichkeit gelegt und die darunter zunehmend leidende Qualität der medizinischen Versorgung auf die Leistungserbringer abgewälzt [23].

Dies war allerdings sehr kurz gedacht, denn die medizinische Versorgung entspricht nicht der industriellen Produktion von Waren und Gütern. Dies mussten auch die Kostenträger nach Jahren des Einsparens erkennen und somit rückten zunehmend auch andere Faktoren in den Vordergrund, die vielmehr ein Indikator für Qualität sind, als die reine Wirtschaftlichkeit ansich.

Die strukturelle Spezialisierung auf ein Krankheitsbild oder ein Verletzungsmuster und die damit einhergehende „hohe“ Fallzahl werden mittlerweile als Indikatoren, die auf eine gute Qualität schließen lassen können, herangezogen. Beispielsweise belegen aktuelle Publikationen zur Ergebnisqualität nach Kopf-Hals-Tumortherapie ein signifikant unterschiedliches Gesamtüberleben in Abhängigkeit der Strukturqualität des Behandlungszentrums bei vermeintlich gleicher Therapie. Erst in jüngster Zeit spielen diese Aspekte auch in Studien eine größere Rolle [24].

Weitere Beispiele für eine Verbesserung der Qualität durch eine organisierte und vorgegebene Struktur sind Brustzentren, Verbrennungszentren, Stroke Units oder Cl-Zentren. Die Liste liese sich beliebig für alle Fachbereiche weiterführen. Zu diesem Themenblock gehören natürlich auch die Schlagwörter Mindestanzahl bzw. Mindestmenge, die ein Arzt für die Kostenträger pro Jahr vorweisen sollte, so dass Leistungen überhaupt noch vergütet werden. Dies ist insbesondere im Bereich der plastisch-ästhetischen Nasenchirurgie eminent, denn nur sehr wenige Ärzte machen überhaupt mehr als dreißig komplette innere und äußere Rhinoplastiken pro Jahr. 
Die Kostenträger befinden sich mit den Leistungserbringern dadurch im Spannungsfeld zwischen einer flächendeckenden adäquaten Versorgung der Bevölkerung auf der einen Seite und der bei speziellen Erkrankungs- und Verletzungsbildern erforderlichen und mittlerweile erbrachten hohen Qualität aufgrund der Spezialisierung auf der anderen Seite. Eine Spezialisierung spiegelt mehr Erfahrung und Expertise wider, die z. B. in weniger Nebenwirkungen bzw. Komplikationen oder einem besseren Gesamtüberleben (s. o.) münden.

Dadurch enstehen volkswirtschaftlich langfristig geringere Kosten, bei einer kürzeren Liegezeit und z. T. einer Verschiebung der stationären Leistung in den ambulanten Bereich, der weniger kostenintensiv ist. Somit wären wir wieder bei der Wirtschaftlichkeit, die zu Beginn als primäres Ziel der Kostenträger aufgeführt wurde.

\section{Kontrolle und Sicherung der Qualität}

\subsection{QM-Methoden und -Instrumente}

Wenn ein Qualitätsproblem prinzipiell erkannt ist und konkrete Ziele zu seiner Verminderung vereinbart worden sind, gibt es eine Vielzahl von Methoden des Qualitätsmanagements (QM), mit denen diese Qualitätsziele erreicht werden können [25].

Die inhaltliche und organisatorische Ausgestaltung von QM-Verfahren sollte sich adäquat auf das jeweilige Qualitätsproblem, die vereinbarten Ziele und den jeweiligen Versorgungskontext beziehen. QM-Systeme/-Verfahren weisen in struktureller Hinsicht und konzeptionellem Spektrum oft erhebliche Unterschiede auf, sodass ein direkter Vergleich der Kriterien nur eingeschränkt möglich ist.

Zum Gelingen von QM-Verfahren ist es ferner unerlässlich, geeignete QM-Verfahren anhand ihrer konzeptionellen Ausrichtung und anhand des Stands ihrer wissenschaftlich belegten Effektivität auszuwählen. Qualitätssicherungsinstrumente können entweder alle oder lediglich einzelne Phasen des Qualitätsverbesserungszyklus umfassen [26].

Für das Qualitätsmanagement in der vertragsärztlichen Versorgung kommen als Beispiel derzeit vorrangig folgende Verfahren in Betracht:

- Qualitätszirkel mit Erstellung und/oder Modifikation von Leitlinien

- Ringversuche

- Qualitätsprüfungen im Einzelfall (Stichproben)

- Zweitmeinungsverfahren

- Datenerfassung und Auswertung von Messgrößen (z. B. Komplikationen)

- Strukturierte externe Vergleiche (z. B.: Benchmarking)

- Usw.

Entsprechende Muster oder Verfahren sind im gesamten Bereich der Ästhetik nicht etabliert.

\subsubsection{Aus ärztlicher Sicht}

\subsubsection{Qualitätsziele zur Patientenorientierung aus Sicht der Ärzteschaft}

Die Mehrheit der Regelungen in der ärztlichen Qualitätssicherung betreffen Maßnahmen der Strukturqualität. Sie sind in der Regel in den einzelnen Qualitätssicherungsbereichen durch die Qualifikation des Arztes nach der Aus-, Weiter- und Fortbildung sowie die er- forderlichen Geräte geprägt. Die Richtlinien bestimmen im Detail, welche Qualifikation der Arzt besitzen muss, welche ggf. seine Mitarbeiter oder welche Geräte zulässig sind, um eine bestimmte Leistung geltend machen zu können, sagt aber nichts über die Qualität der medizinischen Leistung aus. Entsprechende Richtlinien fehlen im Bereich der Rhinochirurgie komplett, so dass diese Eingriffe auch von Allgemeinchirurgen usw. durchgeführt werden können. Dies ist in der ästhetischen Chirurgie ein generelles Problem, da es keine ausreichende gesetzliche Regelung gibt, die diese Therapien und Anwendungen definiert und schützt. Entsprechende Berichte in den Medien über Heilpraktiker, die ästhetisch praktizieren, Internisten, die operieren usw. sind hinlänglich bekannt.

In einer großen Anzahl von Qualitätssicherungsbereichen soll durch die Qualitätssicherung auch die Prozessqualität und Ergebnisqualität geprüft und erreicht werden. Die Ergebnisqualität ist durch die Prüfung des Untersuchungs-/Behandlungszieles (Erfolg oder Outcome) charakterisiert.

\subsubsection{Praktische Umsetzung der Patientenorientierung im QM}

\section{Gründliche und verständliche Aufklärung}

Die gründliche und verständliche Aufklärung und Information des Patienten haben im Qualitätsmanagement eine zentrale Bedeutung. Sie bilden die Basis dafür, informierte Entscheidungen treffen zu können. Der Patient, der zum Arzt geht und ohne zu hinterfragen befolgt, was die Autorität im weißen Kittel sagt, wird immer seltener (s. o.). Die Zahl der Patienten nimmt zu, die über diagnostische und therapeutische Maßnahmen und Möglichkeiten aufgeklärt werden wollen, die jeweiligen Implikationen verstehen und letztlich auch mitentscheiden möchten. Dies entspricht der Kernaussage des Konzeptes der gemeinsamen bzw. partizipativen Entscheidungsfindung (shared decison making). Arzt und Patient treffen auf der Basis geteilter Informationen eine gemeinsam verantwortete Entscheidung, sie gehen eine „therapeutische Allianz“ ein [15]. Dies ist im Selbstzahlerbereich und der Ästhetik überdurchschnittlich repräsentiert, da die Patienten die Leistungen aus eigener Tasche bezahlen und eine entsprechende Behandlung und Qualität dadurch einfordern.

Patienten verstehen oft nur $50 \%$ der Informationen, die sie von ihrem Arzt erhalten [27]. Ob ein Patient vermittelte Informationen zu Nutzen und Nebenwirkung vorgeschlagener Behandlungsoptionen versteht, ist davon abhängig, in welcher Form diese vermittelt werden $[28,29]$.

Auch bei einem gründlichen mündlichen Aufklärungsgespräch ist keine Garantie gegeben, dass Patienten die vermittelten Inhalte verstanden haben und sich erinnern. Viele Patienten können sich nach dem Verlassen des Arztzimmers an den Inhalt des Gespräches nicht mehr korrekt erinnern. Deshalb sollten Patienten alles, was sie im Rahmen der Aufklärung über Befunde und mögliche Therapieoptionen, sowie die weitere Versorgung und Betreuung an mündlicher Beratung erfahren, - in komprimierter Form - „schwarz auf weiß“ mitnehmen können [22]. In der Rhinoplastik bieten sich entsprechende bearbeitete Bilder an, die ein möglich anzustrebendes Ergebnis visualisieren. Der Patient muss aber immer darüber informiert werden, dass es sich um einen Entwurf und nicht um ein garantiertes Endergebnis handelt. 
Das Überzeugende daran ist, dass der Patient etwas mit nach Hause nimmt, das extra für ihn erstellt wurde. Dabei sollte Qualität vor Quantität gehen. Eine individuell auf den Patienten abgestimmte Information wirkt professioneller und persönlicher als ein kopierter Plan oder die übliche Informationsbroschüre.

\section{Vermittlung von Behandlungsoptionen}

Evidenzbasierte Entscheidungsgrundlagen für Arzt und Patient setzen ein Verständnis für den Umgang mit Wahrscheinlichkeiten voraus. Keine Untersuchung ist zu einhundert Prozent sicher, keine Behandlung auf der ganzen Linie wirkungsvoll. Bei Entscheidungen müssen sich Arzt und Patient also immer auf Wahrscheinlichkeiten stützen. Die Bewertung von Nutzen und Risiken ist bei Experten und Laien unterschiedlich. Der Experte stützt seine Bewertungsgrundlagen auf eine (durch die entsprechende Studie) klar definierte Patientengruppe, der Patient selbst erwartet eher eine Ja/NeinEntscheidung. Eine weitere Hürde bei der Kommunikation von Nutzen und Risiken stellt der so genannte Framing Effekt dar. Das bedeutet, dass die Entscheidung davon abhängt, ob eine Information/Option positiv oder negativ dargestellt wird.

\section{Qualität der Arzt-Patienten-Kommunikation}

Zur erfolgreichen Gestaltung der Arzt-Patienten-Kommunikation müssen verschiedene Aspekte berücksichtigt werden [30, 31]:

- Schaffung eines störungsfreien Umfeldes

- Aufbau einer Arbeits- und Vertrauensbeziehung

- Empathie

- Konfliktmanagement

- Echtheit der Beziehung

- Berücksichtigung kommunikationswissenschaftlicher Grundlagen

- Erkennen und kommunizieren der eigenen Grenzen

Patientenversorgung

- Ausrichtung der Versorgung an aktuellen fachlichen Standards und Leitlinien

- Patienten-Orientierung, -Sicherheit, -Mitwirkung, -Information und -Beratung

- Strukturierung von Behandlungsabläufen

Praxisführung/Mitarbeiter/Organisation

- Regelung von Verantwortlichkeiten

- Mitarbeiterorientierung (z. B. Arbeitsschutz, Fort- und Weiterbildung)

- Praxismanagement (z. B. Terminplanung, Datenschutz, Hygiene, Fluchtplan)

- Gestaltung von Kommunikationsprozessen (intern/extern) und Informations-management

- Kooperation und Management der Nahtstellen der Versorgung

- Integration bestehender Qualitätssicherungsmaßnahmen in das interne Qualitätsmanagement

Dies sind alles Bereiche, die der Rhinopatient auch in der Kommunikation von Befund und Therapieoption zwischen ihm und dem Arzt als wichtig empfindet. Der Patient, der sich mit der plastischästhetischen Korrektur seiner Nase auseinandersetzt ist meistens durch das Internet zumindest informiert, durch die Meinung anderer geprägt und im gewünschten optischen Bild von den Medien und seiner Umgebung mehr beeinflusst, als ihm bewusst ist.

\subsubsection{Leitlinien und Qualitätsmanagement}

Seit Jahren wird in der einschlägigen Literatur auf die enge Beziehung zwischen Leitlinien und Qualitätsmanagement hingewiesen [32]. Leitlinien sind eines der wichtigsten Instrumente des Qualitätsmanagements. Die Integration von Leitlinien in QM-Programme ist eine der effektivsten Leitlinien-Implementierungsmaßnahmen.

Zusätzlich dienen Leitlinien im Rahmen des Qualitätsmanagements als Grundlage für die Arbeit in Qualitätszirkeln [33], als Referenz für Qualitätsziele, Qualitätsmerkmale und Qualitätsindikatoren [19], als Grundlage für Prozess- und Ablaufbeschreibungen sowie als Durchführungsanleitungen, z. B. in Form klinischer Behandlungspfade [34]. Die Einführung eines funktionierenden Qualitätsmanagementsystems kann die Umsetzung von Leitlinien in die Handlungsroutine sichern und so nachweislich zur gewünschten Qualitätsverbesserung führen [35]. Gleichzeitig stellen Leitlinien einen wesentlichen Bestandteil eines modernen Informationsmanagements für die ärztliche Tätigkeit in Klinik und Praxis dar und können dadurch als Grundlage einer gleichberechtigten, gemeinsamen Entscheidungsfindung von Patienten und Arzt dienen (s. o.).

\subsubsection{Aufgaben und Ziele von Leitlinien}

Leitlinien haben darüber hinaus die Aufgabe, das umfangreiche Wissen (wissenschaftliche Evidenz und Praxiserfahrung) zu speziellen Versorgungsproblemen explizit darzulegen, unter methodischen und klinischen Aspekten zu bewerten, gegensätzliche Standpunkte zu klären, unter Abwägung von Nutzen und Schaden das derzeitige Vorgehen der Wahl zu definieren.

Vorrangiges Ziel von Leitlinien ist die Verbesserung der Qualität medizinischer Versorgung durch Wissensvermittlung. Leitlinien sollen darauf zielen, unter Berücksichtigung der vorhandenen Ressourcen, gute klinische Praxis zu fördern und die Öffentlichkeit darüber zu informieren, Entscheidungen in der medizinischen Versorgung auf eine rationalere Basis zu stellen, die Stellung des Patienten als Partner im Entscheidungsprozess zu stärken und die Qualität der Versorgung zu verbessern.

Aus Leitlinien können Zielgrößen und Indikatoren abgeleitet werden, anhand derer die Qualität der medizinischen Versorgung messbar und eine Unterscheidung zwischen gut und verbesserungsbedürftig ermöglicht wird. Solche Qualitätsindikatoren sind ein wesentliches Werkzeug für die Evaluierung von Behandlungsleistungen und -Ergebnissen im medizinischen Alltag, für das interne Qualitätsmanagement und für den externen Qualitätsvergleich.

\subsubsection{Wirksamkeit und Qualität von Leitlinien}

Der günstige Einfluss von Leitlinien auf die Prozess- und Ergebnisqualität im Gesundheitswesen ist wissenschaftlich belegt [35]. Die Wirksamkeit und damit letztlich der Nutzen einer einzelnen Leitlinie hängen aber entscheidend von ihrer Qualität und von ihrer Umsetzung ab. Demnach werden heute international bestimmte Kriterien, die hochwertige Leitlinien erfüllen sollten, in einheitlicher Weise definiert [32]. Für den deutschen Raum sind diese Kriterien methodischer Qualität in Form einer kommentierten Checkliste 
publiziert, dem Deutschen Leitlinien-Bewertungs-Instrument (DELBI) [36].

DELBI soll einerseits Leitlinienanwendern und anderen Interessierten helfen, Leitlinien einer methodischen Überprüfung zu unterziehen und damit ihre Validität zu beurteilen. Andererseits kann für Leitlinienautoren die Orientierung an DELBI sicherstellen, dass Leitlinien den internationalen Standards entsprechen.

Dabei sind zunächst drei grundlegende Aspekte hervorzuheben:

- Zusammensetzung des Leitliniengremiums: Repräsentativ für den Anwenderkreis

- Evidenzbasierung: systematische Suche, Auswahl und Bewertung der Literatur

- Methodik der Entwicklung: systematische Evidenz- und Konsensbasierung

Vor allem die Evidenzbasierung ist maßgeblich für die wissenschaftliche Legitimation einer Leitlinie, während die Beteiligung der Anwender sowie die strukturierte Konsensfindung v. a. für die Akzeptanz und Umsetzung entscheidend sind. Um Leitliniennutzern eine Orientierung über das Ausmaß der Berücksichtigung dieser Aspekte zu ermöglichen, werden 4 Klassen von Leitlinien unterschieden (siehe Abb. 2) [37]. Aufgabe des praktizierenden Arztes und Voraussetzung für die Nutzung von Leitlinien im Rahmen der evidenzbasierten Medizin ist die Fähigkeit, gute (d. h. valide, aktuelle, praktikable) Leitlinien zu identifizieren.

\section{Rechtliche Aspekte von Leitlinien}

Leitlinien sind im Gegensatz zu Richtlinien nicht verbindlich [38]. Die Anwendbarkeit einer bestimmten Empfehlung in der individu- ellen Situation ist unter Berücksichtigung der vorliegenden Gegebenheiten (z. B. Begleiterkrankungen des Patienten, verfügbare Ressourcen) zu prüfen. Die nicht rechtliche Bindung wurde 2014 seitens des Bundesgerichtshofs (BGH Urt. V. 15.04.2014 - VI ZR 382/12) bestätigt, insbesondere da Leitlinien nach Ansicht des BGH kein Sachverständigengutachten ersetzen würden. Generell muss der praktizierende Arzt über die für sein Berufsfeld relevanten Leitlinien informiert sein. Er sollte weiterhin ein begründetes Abweichen von Leitlinien-Empfehlungen im Einzelfall im Rahmen der Patientendokumentation festhalten. Das bloße Differieren von einer Leitlinie wird kaum als fahrlässiges Verhalten ausgelegt werden, es sei denn, die betreffende Vorgehensweise ist so gut etabliert, dass kein verantwortlicher Arzt sie außer Acht lassen würde. Dies bedeutet aber nicht, dass eine Leitlinie, selbst wenn sie für die Feststellung fahrlässigen Handelns nicht maßgebend ist, in einem Gerichtsverfahren keine weiteren Konsequenzen haben könnte. Zum Beispiel kann sie zu einer Umkehr der Beweislast führen, d. h. hat ein Arzt eine Leitlinie nicht befolgt, wird von ihm möglicherweise der Nachweis verlangt, dass der dem Patienten zugefügte Schaden nicht durch das Nichtbefolgen der Leitlinie entstanden ist.

Für gewöhnlich hängt der Stellenwert, den Leitlinien vor Gericht einnehmen, von mehreren Faktoren ab, insbesondere davon, inwieweit sie wissenschaftlich fundiert sind, einen Expertenkonsens darstellen und von einer dazu autorisierten Gruppe oder Institution herausgegeben wurden. Grundsätzlich werden Leitlinien keine endgültigen Antworten geben, auch wenn sie im Hinblick auf ihre Anwendung nur wenig Flexibilität gestatten. Denn Medizin ist im Fluß, entwickelt sich mit immer schnellerem Tempo weiter, sodass jede einzelne Handlungsmaxime im Lichte des speziellen Gesundheits-

\begin{tabular}{|c|c|c|c|}
\hline Bezeichnung & Charakteristika & $\begin{array}{l}\text { Wissenschaftliche } \\
\text { Legitimation der } \\
\text { Methode }\end{array}$ & $\begin{array}{l}\text { Legitimation } \\
\text { für die } \\
\text { Umsetzung }\end{array}$ \\
\hline $\begin{array}{l}\text { S1: } \\
\text { Handlungsempfehlung } \\
\text { von Experten }\end{array}$ & $\begin{array}{l}\text { Konsensfindung in einem informellen } \\
\text { Verfahren }\end{array}$ & gering & gering \\
\hline $\begin{array}{l}\text { S2K: } \\
\text { Konsensbasierte } \\
\text { Leitlinien }\end{array}$ & $\begin{array}{l}\text { Repräsentatives Gremium, } \\
\text { strukturierte Konsensfindung }\end{array}$ & gering & hoch \\
\hline $\begin{array}{l}\text { S2e: } \\
\text { Evidenzbasierte } \\
\text { Leitlinien }\end{array}$ & $\begin{array}{l}\text { Systematische Recherche, Auswahl, } \\
\text { Bewertung der Literatur }\end{array}$ & hoch & gering \\
\hline $\begin{array}{l}\text { S3: } \\
\text { Evidenz- und } \\
\text { Konsensbasierte } \\
\text { Leitlinien }\end{array}$ & $\begin{array}{l}\text { Repräsentatives Gremium, } \\
\text { systematische Recherche, Auswahl, } \\
\text { Bewertung der Literatur, strukturierte } \\
\text { Konsensfindung }\end{array}$ & hoch & hoch \\
\hline
\end{tabular}

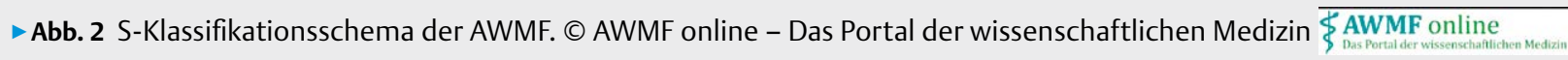


problems sowie der besonderen Umstände des jeweiligen Patienten beurteilt werden muss. Manchmal existieren konkurrierende, z. B. in unterschiedlichen Krankenhäusern oder Regionen erstellte Leitlinien; in anderen Fällen können gutachterliche Aussagen vor Gericht dazu benutzt werden, die Autorität einer Leitlinie in Frage zu stellen. Aus all diesen Gründen werden die Gerichte die Befolgung von Leitlinien nicht automatisch mit guter medizinischer Praxis gleichsetzen [36].

\subsubsection{Nutzung von Leitlinien im Qualitätsmanagement - Leit- linien-Implementierung}

Die Erstellung und Veröffentlichung von Leitlinien allein ist nicht hinreichend, um ihre Anwendung im Versorgungsalltag zu sichern. Man versteht unter Leitlinienimplementierung den Transfer von Handlungsempfehlungen in individuelles Handeln bzw. Verhalten von Ärzten, Patienten, Betroffenen usw.

Um diesen Transfer erfolgreich zu gestalten, müssen im Allgemeinen verschiedene, sich ergänzende Maßnahmen vorgenommen werden, die zielgerichtet auf die identifizierten Problemfelder abgestimmt sind. Dabei handelt es sich um edukative, finanzielle, organisatorische und/oder regulatorische Strategien [39].

Entscheidend für die Frage der Implementierung ist nicht nur die reine Verfügbarkeit einer Leitlinie, sondern, dass sich die Anwender die Inhalte der Leitlinie zu Eigen machen und diese an ihre individuellen Bedürfnisse und Gegebenheiten anpassen. Im ambulanten Bereich existieren dazu erfolgreiche Beispiele solcher Adaptationen im Rahmen der Qualitätszirkelarbeit [40,41].

\subsubsection{Umsetzung von Leitlinien in der ambulanten Versor- gung}

Die Berücksichtigung evidenzbasierter Leitlinien ist mittlerweile in mehreren vertragsärztlichen Versorgungsprogrammen gesetzlich verpflichtend, und zwar im Rahmen der Strukturierten Behandlung bei chronischen Krankheiten nach § 137f SGB V sowie seit der Gesetzesnovellierung im April 2007 bei der „Hausarztzentrierten Versorgung“ (HZV) nach § 73b SGB V.

Strukturierte Behandlungsprogramme müssen auf einheitliche Therapie-Empfehlungen gestützt werden, für die in Deutschland die notwendigen evidenzbasierten Konsensusleitlinien bislang noch nicht zu allen relevanten Erkrankungen zur Verfügung stehen. Vor diesem Hintergrund hat die Bundesärztekammer im Frühjahr 2002 das sogenannte Nationale Programm für Versorgungsleitlinien (NVL) „initiiert und die ersten Versorgungsleitlinien (Asthma, COPD, Typ-2-Diabetes und KHK) publiziert. Ziel dieses gemeinsam von BÄK, KBV und AWMF getragenen Programms ist die Implementierung von evidenzbasierten, deutschen Leitlinien im Rahmen der strukturierten Krankenversorgung. Die Empfehlungen geben den interdisziplinären Konsens aller an der Versorgung einer bestimmten Patientengruppe beteiligten medizinischen Fachgesellschaften und Disziplinen auf der Grundlage der besten verfügbaren Evidenz wieder. Das Programm für NVL beinhaltet gezielte Instrumente und Maßnahmen zur Verbreitung und Implementierung der NVL, um eine effektive Umsetzung in der Praxis zu ermöglichen. Ein Fokus liegt dabei auf den Bereichen Qualitätsmanagement, Qualitätsindikatoren, und Fortbildung. Falls sich insbesondere die Etablierung von NVLs auch monetär auswirken und dies nachzuweisen ist, ist davon auszugehen, dass Gesetzgeber und Kostenträger darauf drängen werden diese für alle Bereiche zu etablieren. Die Gefahr einer Einschränkung der ärztlichen Therapiefreiheit steht hierbei im Raum.

Medizinische Fachinformationen für Laien sollten im Kontext zu bestehenden ärztlichen Leitlinien stehen und den Ratsuchenden eine Hilfestellung bei der Entscheidungsfindung während aller Phasen der medizinischen Betreuung (Diagnostik, Therapie, Nachbetreuung) geben. Die Informationen sollten valide Daten auf der Basis der evidenzbasierten Medizin bieten. In diesem Zusammenhang sind Patienteninformationen erfolgreiche Instrumente der Leitlinienimplementierung.

\subsubsection{Leitlinien in der Rhinoplastik}

Die für die Rhinoplastik wesentliche Leitlinie (AWMF-Register Nr. 017/070) wurde im Juni 2000 erstellt, im Januar 2016 überarbeitet, entspricht dem Standard S2k und steht zur erneuten Überprüfung für Januar 2021 an. Sie wurde als Konsensusarbeit im Auftrag der DGHNOKHC von 11 ärztlichen Kollegen aus den drei Fachbereichen HNO, Plastische Chirurgie und MKG erarbeitet. Sie erfüllt somit die DELBI Kriterien der Repräsentanz des Anwenderkreises (HNO, Plastische Chirurgie, MKG), ist strukturiert konsensusbasiert (S2k) und in der Entwicklung (Kontrolle 2021).

Es heisst in der Zielbeschreibung der Leitlinie zu Beginn: „Ziel dieser Leitlinie ist die Förderung einer qualitativ hochwertigen fachärztlichen Versorgung von Patienten mit Formstörung der inneren und äußeren Nase, die mit einer funktionalen Beeinträchtigung oder einer relevanten ästhetischen Beeinträchtigung einhergehen. Dies soll in besonderem Maße zur Reduktion der assoziierten krankheitsbedingten Morbidität, zu einem rationellen Einsatz diagnostischer und therapeutischer Verfahren sowie zur Reduktion der krankheitsbedingten sozioökonomischen Faktoren beitragen. Angestrebt werden eine sinnvolle angemessene Diagnostik und Therapie auf dem derzeitigen Stand fachlicher Erkenntnisse“.

Diese Zielbeschreibung ist essentiell, denn hierdurch wird auch die Mindestqualitätsanforderung aus ärztlicher Sicht definiert, die für die Korrektur der inneren und äußeren Nase bzw. der funktionellen und ästhetischen Komponenten von wesentlicher Bedeutung sind. Sie sollen den Behandler sprichwörtlich „leiten“, um für den Patienten das beste Konzept unter Leitlinienqualität zu entwickeln. Die S2k Leitlinie Rhinochirurgie hat eine hohe Legitimation in der Umsetzung aufgrund des repräsentativen Gremiums und der strukturierten Konsensusfindung ist allerdings wissenschaftlich gering legitimiert, da leider keinerlei Evidenz. Bei S2e ist es genau umgekehrt (siehe $>$ Bild 2). Eine Steigerung in der Klassifikation ist langfristig unbedingt anzustreben, um die plastisch-ästhetische Nasenchirurgie als S3 Leitlinie, wie beim Larynxkarzinom zu etablieren. Dies kann natürlich auch bedeuten, dass „liebgewonnene“ Vorgehensweisen plötzlich einer wissenschaftlichen Analyse nicht mehr standhalten können und umgedacht werden muss. Dieser Herausforderung können sich aber alle Rhinochirurgen stellen, insbesondere wenn man die Veränderungen der OP-Techniken in den letzten 50 Jahren sieht (Abb. 2) und dies zu einer deutlich verbesserten Versorgung der Patienten geführt hat. Dies sollte ebenfalls das Bestreben aller Ärzte sein, ansonsten hätten wir keinen medizinischen Fortschritt von dem alle profitieren und würden unsere Patienten immer noch wie vor vielleicht 50 Jahren behandeln. 
Der differenzierte Patient greift ebenfalls auf Leitlinien zurück und konfrontiert gelegentlich den Behandler, ob sein Vorgehen „Leitlinienkonform“ sei. Sie sind wesentlicher Bestandteil in der täglichen Arbeit des Rhinochirurgen, sollten ihm geläufig sein und haben auch aus ästhetischer Sicht eine wesentliche Bedeutung, die explizit aus Patientensicht eingefordert wird. Allerdings muss interessanterweise berücksichtig werden, dass die Lebensqualität in der rhinochirurgischen Leitlinie nur im Bereich der Riechfunktion und nicht in den anderen Bereichen (Atmung, Optik usw.) der Nase erwähnt wird. Dies sollte vielleicht ferner ein Ansinnen für die Überprüfung der Leitlinie ab dem Jahr 2021 sein.

\subsubsection{Qualität und Patientensicherheit}

Die Prävention von unerwünschten Ereignissen ist das Ziel aller Aktivitäten im Bereich der Patientensicherheit. Entscheidend hierfür ist die in der jeweiligen Institution vorherrschende Sicherheitskultur. Diese Sicherheitskultur kann durch spezifische Maßnahmen und durch das Engagement Einzelner aufgebaut und etabliert werden. Es besteht ein Wechselspiel zwischen Sicherheitskultur und Maßnahmen zur Verbesserung der Patientensicherheit. Unter Sicherheitskultur in der Medizin versteht man, dass eine Organisation (z. B. Arztpraxis) dauerhaft und auf allen Ebenen danach strebt, dass Patienten keine (vermeidbaren) unerwünschten Ereignisse durch die Gesundheitsversorgung davontragen.

\subsubsection{CIRS medical}

CIRSmedical ist das Berichts- und Lernsystem der deutschen Ärzteschaft für kritische Ereignisse in der Medizin. Die Buchstaben „CIRS“ stehen für Critical Incident Reporting-System. Es ist anonym, sicher und ermöglicht gegenseitiges Lernen aus Fehlern und kritischen Ereignissen. Es richtet sich an die Mitarbeiterinnen und Mitarbeiter des Gesundheitswesens. Alle sicherheitsrelevanten Ereignisse, die in der Medizin auftreten, können von Mitarbeiterinnen und Mitarbeitern des Gesundheitswesens berichtet werden. Dies können Fehler, Beinahe-Schäden, kritische Ereignisse oder auch unerwünschte Ereignisse sein. Als Beispiele seien der „Manchester Patient Safety Framework“ und der „Safety Attitudes Questionaire" genannt.

Die Berichte dürfen keine Daten enthalten, die Rückschlüsse auf die beteiligten Personen oder Institutionen erlauben (Namen, Ortsangaben oder ähnliches) [42, 43]. Im Bereich der HNO bzw. Plastischen Chirurgie sind dem Verfasser solche fokussierten Erhebungsbögen nicht bekannt.

Aus Sicht der plastisch-ästhetischen Nasenchirurgie wäre es vielleicht ein Ansatz unerwünschte oder kritische Ereignisse im Rahmen der Operation und Nachsorge dort anonym zu bündeln, auszuwerten, zu diskutieren, um ggf. entsprechende Lehren ziehen zu können, die sich dann möglicherweise langfristig in der Leitlinie wiederfinden. Diese Informationen sollten auf alle Fälle für jedes Fachpersonal zugänglich sein.

\subsubsection{Aus Patientensicht}

Aus Patientensicht greifen die letzten Abschnitte des letzten Kapitels direkt in dieses über. QM/QS, Fragebögen und auch die Meldung von „kritischen“ Ereignissen werden von Patienten als positiv bewertet. Denn die Patientinnen und Patienten wissen, dass Medi- zin ein hochsensibler Bereich ist, in dem Fehler passieren können. Deswegen kommt es darauf an, wie man, im Interesse der Patienten, mit diesen umgeht.

\subsubsection{Patientenbefragung im Qualitätsmanagment}

Patientenbefragungen stellen ein wichtiges Instrument dar, um Rückmeldungen zur Zufriedenheit der Patienten und Anregungen für Verbesserungen zu erhalten. Sie sollen möglichst mit validierten Fragebögen und soweit möglich regelmäßig durchgeführt werden.

Der Fragebogen „Zufriedenheit in der ambulanten Versorgung - Qualität aus Patientenperspektive“ (ZAP) ist für die anonyme, schriftliche Befragung von volljährigen Patienten entwickelt worden. Patienten, die sich in haus- oder fachärztlicher Behandlung befinden und die Praxis kennen, d. h. mindestens zwei Behandlungskontakte hatten, können mithilfe des Fragebogens die jeweilige Praxis in folgenden Bereichen bewerten:

Praxisorganisation, Information, Interaktion, Fachkompetenz, Einbindung in Entscheidungsprozesse, Vertrauen, Behandlungsqualität und allgemeine Zufriedenheit mit dem Arzt/ der Ärztin. Hier ist deutlich zu sehen, wie sich die sogenannten „harten“ und „weichen“ Faktoren aus dem Kapitel 3.1.2 wiederfinden und seitens des G-BA etabliert wurden.

\subsubsection{Patientenbeschwerden als Instrument im QM}

Eine Beschwerde ist nicht gleichzusetzen mit einem Vertrauensbruch zwischen Arzt und Patient. Vielmehr kann sie eine wesentliche Informationsquelle zur Verbesserung der Qualität in der Behandlung sein. Patientenbeschwerden sind wichtige Rückkoppelungen an alle in der medizinischen Behandlung; daher sind Beschwerden anzuhören, zu bewerten, und es ist auf sie einzugehen. Der behandelnde Arzt muss auch bei Beschwerdefällen oder Vorschlägen Ansprechpartner des Patienten sein.

Bei individuellen Patientenbeschwerden geht es in erster Linie darum, die vom Patienten identifizierten Nöte, Ängste, Sorgen, Ärgernisse usw. aufzunehmen und im individuellen Fall weiter zu helfen.

Wenn es darum geht, ein Meinungsbild der Patienten als Unterstützung des Qualitätsmanagements in der Behandlung zu gewinnen, gibt es z. B. folgende Möglichkeiten: Erfassung von Erfahrungen und Zufriedenheit durch individuelle Rückmeldungen (z. B.: Patientenbox) oder standardisierte Rückmeldungen durch systematische Patientenbefragungen. Da sich Patientenbewertungen aus verschiedenen Dimensionen zusammensetzen, wie den Erwartungen und Bedürfnissen, den Vorstellungen und Annahmen bezüglich des Ergebnisses einer Behandlung, der persönlichen Einstellungen, der sozialen Herkunft, der Bildung, der realen Erfahrungen in einer Situation und dem gesundheitlichen Ergebnis, macht es auch Sinn, diese Parameter zu erheben.

In der Nasenchirurgie ist dies ein Bereich, der nicht nur in den Vorgesprächen, sondern explizit in der Nachsorge auf jeden Operateur zukommt. Ausdrücklich seien hier die Faktoren Erwartungen und Bedürfnisse sowie Vorstellungen und Annahmen des Patienten genannt, die nicht selten diametral zum Ergebnis oder der Wahrnehmung des Arztes stehen können. Selbst wenn präoperativ, seitens des Operateurs, schon ausdrücklich die Grenzen einer Behandlung und eines Ergebnisses dem Patienten erläutert wurden bzw. der Ausgangsbefund nur begrenzte Möglichkeiten offenlässt, prallen diese mit den Erwartungen, Wünschen, Annahmen 
und Vorstellungen des Patienten postoperativ zusammen, wobei die soziale Herkunft, das Umfeld und die Bildung wesentliche Punkte sind, die das beeinflussen (siehe Kapitel 12) [44].

Aufgrunddessen ist es wesentlich die Fragen eindeutig zu formulieren und zahlreiche weitere, in der Literatur zur Entwicklung von Erhebungsinstrumenten zusammengefasste Aspekte zu berücksichtigen. Es empfiehlt sich bereits vorliegende, validierte Fragebögen zu nutzen, die gegebenenfalls im Hinblick auf die Bedürfnisse und Zielsetzungen der jeweiligen Behandlung unter fachkundiger Anleitung angepasst werden können [45].

\subsubsection{Aus Kostenträgersicht}

Aufgrund des zunehmenden Kostendrucks aber auch der deutlicher werdenden Ansprüche von Patienten hinsichtlich einer verbesserten Qualität bei transparenten Gesundheitsinformationen (Stichwort Internet) wurden verschiedene Institute seit Beginn der 2000er Jahre mit dem Ziel der Qualitätsfeststellung und Qualitätssicherung etabliert. Hierunter seien 2 genannt, die direkt bzw. indirekt auf die medizinische Versorgung und Behandlung Einfluss nehmen.

\subsubsection{Institut für Qualität und Wirtschaftlichkeit im Gesund- heitswesen (IQWiG)}

Qualität und Wirtschaftlichkeit - das sind aus Kostenträgersicht 2 entscheidende Faktoren für ein gutes und leistungsfähiges Gesundheitswesen (s. o.). Um dieses Ziel zu erreichen ist es wichtig, die Vor- und Nachteile medizinischer Leistungen für die Patienten objektiv zu überprüfen. Das IQWiG hat unter anderem den gesetzlichen Auftrag, Vor- und Nachteile von medizinischen Verfahren zu bewerten, also z. B. verschiedene Arzneimittel oder Operationsverfahren untereinander zu vergleichen. Das IQWiG führt keine eigenen klinischen Studien mit Patientinnen und Patienten durch. Vielmehr sucht das Institut aus den vorhandenen Studien systematisch diejenigen heraus, in denen die gefragten Vergleiche beschrieben sind und die ausreichend evidenzbasierte und verlässliche Ergebnisse liefern. Aus dieser systematischen Recherche entsteht dann ein zusammenfassendes Gutachten für den Auftraggeber. Es publiziert alle Ergebnisse auf seinen Webseiten und richtet sich damit sowohl an Fachleute aus dem Gesundheitswesen als auch direkt an alle Bürger. Aufträge darf das IQWiG ausschließlich vom Gemeinsamen Bundesausschuss oder vom Bundesministerium für Gesundheit annehmen. Die Ergebnisse werden als Berichte oder Dossierbewertungen bzw. Potenzialbewertungen veröffentlicht. Das Institut kann aber auch in eigener Regie Fragen von grundlegender Bedeutung aufgreifen und bearbeiten. Das IQWiG erstellt somit Berichte über den Nutzen oder (Zusatz-)Nutzen von medizinischen Maßnahmen aus medizinischer und kostentechnischer Sicht und kann hieraus Empfehlungen abgeben. Das IQWiG trifft aber nicht die Entscheidung, ob die Kosten einer Leistung von den Krankenkassen erstattet werden. Das entscheidet alleine der G-BA.

\subsubsection{Institut für Qualitätssicherung und Transparenz im Ge-} sundheitswesen (IQTiG)

Im Jahr 2014 wurde das IQTiG im Rahmen des Gesetzes zur Weiterentwicklung der Finanzstruktur und der Qualität in der gesetzlichen Krankenversicherung (GKV-FQWG) als fachlich unabhängiges, wissenschaftliches Institut für Qualitätssicherung und Trans- parenz im Gesundheitswesen auf Basis von §137a SGB V durch den G-BA gegründet. Es entwickelt, entsprechend des Krankenhausstrukturgesetzes, im Auftrag des G-BA Konzepte für planungsrelevante Qualitätsindikatoren sowie Zu- und Abschläge in der qualitätsorientierenden Vergütung und evaluiert die Qualitätsverträge nach §110a SGB V. Neben der Erarbeitung von Instrumenten der Qualitätssicherung soll die Fortführung und Weiterentwicklung der bereits existierenden Qualitätssicherungsverfahren garantiert werden. Die Entwicklung und Durchführung von Verfahren, die externe Qualitätssicherung der Versorgung, die Schaffung von Kriterien zur Bewertung von Zertifikaten und Qualitätssiegeln sowie die Publizierung der Ergebnisse soll Krankenhäuser hinsichtlich ihrer Qualität miteinander vergleichbar machen. Im Erfassungsjahr 2019 führte das IQTIG insgesamt 24 QS-Verfahren in den Gebieten Viszeral- bzw. Gefäßchirurgie, Kardiologie und Herzchirurgie, Transplantationsmedizin, Hygiene und Infektionsmanagment, Gynäkologie, Perinatalmedizin, Orthopädie und Unfallchirurgie sowie der Pflege durch.

Das QS-Verfahren soll die qualitätsrelevanten Aspekte im Bereich von Komplikationen bzw. unerwünschten Ereignissen, erneuten Eingriffen aufgrund von Komplikationen und des Überlebens der Patienten messen, vergleichend darstellen und bewerten. Ziele des Verfahrens sind insbesondere die Verbesserung der Durchführung der Eingriffe zur Erhöhung der Patientensicherheit, die Verringerung der Komplikationsraten während und nach den Eingriffen sowie die Verringerung von Folgeerkrankungen durch die Eingriffe und somit eine Reduktion der Kosten.

\subsubsection{Qualität und Kosten am Beispiel der Septorhinoplastik} Die Kostenträger analysieren einzelne Operationsmethoden anhand der Qualitätsangepassten Lebensjahre (QALY = quality-adjusted life years). Es wird durch Erfassung der subjektiven Einschätzung der Patienten vor und nach der Operation durch sogenannte PROMs (siehe Teil 2, Kapitel 12) eine Verbesserung oder Verschlechterung der Lebensqualität eingeschätzt um hieraus eine Kosten-/ Nutzenanalyse zu errechnen. Sie bildet sich aus dem Quotienten der Kosten geteilt durch den Zugewinn der errechneten Lebensqualität. Analysen im Bereich der Septorhinoplastik ergeben im Mittel einen QALY-Zuwachs von 0,04 Punkten [46]. Die deutlich höheren publizierten Werte von 0,08 waren immer einer Kombinationsoperation aus Nase inklusive der Nebenhöhlen geschuldet [47]. Damit liegt die QALY im Bereich der Rhinochirurgie sehr gut, verglichen mit anderen Eingriffsbereichen, wie der Otologie 0,01 [48] oder der Cl-Versorgung 0,035 [49]. Ausserhalb der HNO lassen sich höhere Werte nur im Bereich Herzkatheter und Hüfttotalendoprothese nachweisen $(0,1)$ [50].

Bei einem Landesbasisfallwert von $€ 3539,12$ im Jahre 2019 für Baden-Württemberg und einer Bewertungsrelation von 1,139 bei komplexen Septorhinoplastiken ergibt sich ein Erlös von bis zu $€ 4031,06$. Wenn man dies nun in Relation zum QALY-Zuwachs von 0,04 setzt, ergibt sich die ICUR (incremental cost utiliy ratio) mit Kosten von ca. $€ 100000$ pro dazugewonnenem QALY. Dieser hohe ICUR liegt nicht an der teuren Therapie, sondern eher an dem niedrigen QALY Zuwachs. Kostenträger werden zunehmend diese Berechnungen ins Spiel bringen und fokussieren, um rein betriebswirtschaftlich einen Eingriff rechtfertigen zu können oder nicht. Entsprechende Entwicklungen gibt es schon in Großbritannien, wo 
bestimmte Eingriffe ab einem gewissen Alter nicht mehr von der Krankenkasse übernommen werden (z. B. Hüfte) oder diese nur noch auf Einzelfallentscheidungen beruhen (z. B. Septorhinoplastik) [51]. Das heißt im Umkehrschluß, dass aus Sicht der Kostenträger die Kosten des Eingriffs geringer werden müssen (niederere DRG), oder dass die gewonnenen Lebensqualitätsjahre bei unveränderten Kosten deutlich ansteigen müssten. Dabei ist immer zu beachten, dass die QALY die subjektive Empfindung des Patienten wiedergibt und nicht das objektive Ergebnis. Die ärztliche Qualität spielt somit nur indirekt eine Rolle, da wesentliche demografische Merkmale Einfluss auf die Patientensicht nehmen, die ausserhalb der Behandlung stehen und die prä- und postoperative Analyse beeinflussen (siehe Teil 2, Kapitel 12) [44].

\section{Evidenzbasierte Medizin}

\subsubsection{Definition und Hintergrund}

Die Idee der evidenzbasierten Medizin lässt sich auf das in der 2. Hälfte des im 18. Jahrhundert von britischen Ärzten entwickelte Konzept der „medical arithmetic“ zurückführen [52]. Erstmalig findet sich die Bezeichnung in dem 1793 publizierten Artikel „An attempt to improve the Evidence of Medicine“ des schottischen Arztes George Fordyce [53]. In Großbritannien wurde auch eine der ersten kontrollierten klinischen Studien von James Lind durchgeführt, der schon 1747 die Ergebnisse seines Versuchs, den Skorbut bei Seemännern mit Orangen und Zitronen zu behandeln, veröffentlichte.

Im deutschsprachigen Raum kommt, dem in Wien tätigen, ungarischen Arzt Ignaz Semmelweis (1818-1865) die Erstautorenschaft für die Einführung der „systematischen klinischen Beobachtung" in die medizinische Forschung zu (1848).

Das 1972 erschienene Buch „Effectiveness and Efficiency: Random Reflections on Health Services“ von Professor Archie Cochrane, einem britischen Epidemiologen, markiert den Beginn der aktuellen internationalen Bemühungen um „Evidence-based Medicine“. Seine weiteren Arbeiten führten zu einer zunehmenden Akzeptanz von klinischer Epidemiologie und kontrollierten Studien. Cochrane wurde dadurch gewürdigt, dass ein internationales Netzwerk zur Wirksamkeitsbewertung in der Medizin - die Cochrane Collaboration - nach ihm benannt wurde [54]. Trotz ihrer historischen Wurzeln ist die Evidenzbasierte Medizin eine junge Disziplin, deren positive Wirkungen gerade erst nachgewiesen werden und die sich rasant weiterentwickelt. Diese Evolution ist im vollen Gange und wird insbesondere unter dem Aspekt der Digitalisierung und dem weltweiten Sammeln von und Zugriff auf Daten sowie der Begriffe „Evidence based und precision medicine“ ganz neue Dimensionen erreichen [55].

Um mit den Worten David Sacketts, dem Pionier der der klinischen Epidemiologie und wissenschaftlich fundierten Patientenversorgung, die sich seit den 1990er Jahren unter dem Begriff der Evidenzbasierte Medizin (EbM) etablierte, zu sprechen:

„EbM ist der gewissenhafte, ausdrückliche und vernünftige Gebrauch der gegenwärtig besten externen, wissenschaftlichen Evidenz für Entscheidungen in der medizinischen Versorgung individueller Patienten. Die Praxis der EbM bedeutet die Integration individueller klinischer Expertise mit der bestverfügbaren externen Evidenz aus systematischer Forschung" [56].
Mit individueller klinischer Expertise meint er das Können und die Urteilskraft, die Ärzte durch ihre Erfahrung und klinische Praxis erwerben. Ein Zuwachs an Expertise spiegelt sich auf vielerlei Weise wider, besonders aber in treffsichereren Diagnosen und in der mitdenkenden und -fühlenden Identifikation und Berücksichtigung der besonderen Situation, der Rechte und Präferenzen von Patienten bei der klinischen Entscheidungsfindung im Zuge ihrer Behandlung (siehe Kapitel 3.1.2).

Mit bester verfügbarer externer Evidenz beschreibt er die klinisch relevante Forschung, oft medizinische Grundlagenforschung, aber insbesondere die patientenorientierte Forschung zur Genauigkeit diagnostischer Verfahren (einschließlich der körperlichen Untersuchung), zur Aussagekraft prognostischer Faktoren und zur Wirksamkeit und Sicherheit therapeutischer, rehabilitativer und präventiver Maßnahmen. Externe klinische Evidenz führt zur Neubewertung bisher akzeptierter diagnostischer Tests und therapeutischer Verfahren und ersetzt sie durch solche, die wirksamer, genauer, effektiver und sicherer sind.

Externe klinische Evidenz kann individuelle klinische Erfahrung zwar ergänzen, aber niemals ersetzen und umgekehrt. Es ist gerade diese individuelle Expertise, die entscheidet, ob die externe Evidenz überhaupt auf den einzelnen Patienten anwendbar ist und, wenn das zutrifft, wie sie in die Entscheidung integriert werden kann. In gleicher Weise müssen jede Leitlinie und jedes Vorgehen dahingehend überprüft werden, ob und wie es den klinischen Zustand des Patienten verbessern.

EbM ist definitiv keine Kochbuchmedizin. EbM bedarf von seiner Ausrichtung her eines sogenannten „Bottom-up“-Ansatzes, der die beste verfügbare externe Evidenz mit individueller klinischer Expertise und Patientenpräferenzen verbindet. Das heißt die Grundidee besteht immer darin zunächst abgegrenzte, detaillierte Teilprobleme zu lösen, mit deren Hilfe dann größere, darüber liegende Probleme usw. gelöst werden. Die einzelnen Teillösungen werden von unten (bottom) nach oben (up) zusammen-gesetzt, bis das Gesamtproblem gelöst ist. Schlussfolgernd ist das EbM-Konzept nicht mit dem sturen Befolgen eines Kochrezeptes zur Patientenbehandlung vereinbar.

Es gibt aber auch kritische Stimmen, die befürchten, daß die EbM von Kostenträgern und der Politik instrumentalisiert wird, um die Kosten im Gesundheitswesen zu reduzieren (s. o.). Dies wäre nicht nur ein Mißbrauch des Konzeptes, sondern auch ein fundamentales Mißverständnis der finanziellen Konsequenzen. Ärzte, die EbM substanziell praktizieren, werden die effektivsten Verfahren identifizieren und anwenden, um die Lebensqualität und -dauer der Patienten zu maximieren. Dies kann damit eher zu einer Erhöhung als zu einer Reduktion der Kosten führen.

EbM stützt sich auf die drei Säulen der individuellen klinischen Erfahrung, der Werte und Wünsche des Patienten und des aktuellen Standes der klinischen Forschung.

Das Vorgehen in der EbM gliedert sich in 5 Schritte:

1. Übersetzung des klinischen Problems in eine Fragestellung, die durch wissenschaftliche Untersuchungen zu beantworten ist

2. Systematische Literaturrecherche nach geeigneten Studien

3. Kritische Evidenzbewertung über alle identifizierten Studien hinwe

4. Anwendung der gewonnenen Einsichten in Abwägung der konkreten klinischen Situation 
5. Selbstkritische Evaluation und ggf. Anpassung der bisherigen Vorgehensweise

Das fünfschrittige Vorgehen bedarf des Trainings und ist im Alltag der Patientenversorgung häufig nicht praktikabel. Wesentlich für die EbM ist die systematische, zeitnahe und unverzerrte Berücksichtigung von Studienergebnissen (s. o.).

\subsubsection{Evidenzklassen}

Die evidenzbasierte Medizin sollte als eine Hilfestellung für verschiedene therapeutische Fragestellungen verstanden werden, die ausreichend Spielraum für die Einbindung eigener klinischer Erfahrungen lassen. EbM ist nicht nur auf randomisierte, kontrollierte Studien und Metaanalysen begrenzt. Sie beinhaltet ebenfalls die Suche nach der jeweils besten wissenschaftlichen Evidenz zur Beantwortung der klinischen Fragestellung.

Um etwas über die Genauigkeit eines diagnostischen Verfahrens zu erfahren, benötigt man gut durchgeführte Querschnittsstudien von Patienten, bei denen die gesuchte Krankheit klinisch vermutet wird - keine kontrollierte Studie.

Für eine prognostische Fragestellung benötigen wir methodisch einwandfreie Follow-up-Studien von Patienten, die in einem einheitlichen, frühen Stadium ihrer Krankheit in die Studie aufgenommen wurden. Manchmal finden wir die benötigte Evidenz in Grundlagendisziplinen wie Genetik oder Immunologie.

Insbesondere bei der Frage nach Therapiemethoden sollten wir jedoch nicht-experimentelle Ansätze vermeiden, da diese häufig zu falsch-positiven Schlüssen hinsichtlich der Wirksamkeit von Behandlungen kommen. Da randomisierte, kontrollierte klinische Studien und besonders systematische Übersichten dieser Studien mit höherer Wahrscheinlichkeit korrekte Daten liefern und falsche Schlußfolgerungen weniger wahrscheinlich sind, wurden sie zum „Goldstandard“ für die Beantwortung der Frage, ob Therapiemaßnahmen mehr nützen als schaden. Allerdings sind für manche Fragestellungen keine kontrollierten Studien notwendig (etwa erfolgreiche Interventionen bei sonst fatalen Folgen), oder es bleibt keine Zeit für klinische Studien. Falls keine kontrollierte Studie für die besondere Situation der Patienten durchgeführt wurde, muss die nächstbeste externe Evidenz gefunden und berücksichtigt werden.

Dabei unterscheidet man nach den Empfehlungen des AHRQ (Agency for Healthcare Research and Quality) die Evidenzklassen I bis V und Härtegrade, die die klinische Sicht widerspiegeln. Studien der Klasse la haben die höchste Evidenz, Studien der Klasse V die geringste. Je höher die Evidenzklasse, desto besser ist die wissenschaftliche Begründbarkeit für eine daraus abgeleitete Therapieempfehlung (Härtegrad).

- Stufe la: Wenigstens eine Metaanalyse auf der Basis methodisch hochwertiger RCTs

- Stufe Ib: wenigstens ein ausreichend großer, methodisch hochwertiger RCT

- Stufe Ila: wenigstens eine hochwertige Studie ohne Randomisierung

- Stufe Ilb: wenigstens eine hochwertige Studie eines anderen Typs, (z. B.: experimentelle Studie)

- Stufe III: mehr als eine methodisch hochwertige nichtexperimentelle Studie, wie etwa Vergleichsstudien, Korrelationsstudien oder Fall-Kontroll-Studien
- Stufe IV: Meinungen und Überzeugungen von angesehenen Autoritäten (aus klinischer Erfahrung), Expertenkommissionen, beschreibende Studien

- Stufe V: Fallserie oder eine oder mehrere Expertenmeinungen

Gewichtung und Empfehlung der Evidenzklassen mit Härtegraden (modifiziert nach AHCPR 1992, SIGN 1996)

Härtegrad Evidenzklassen

A la oder Ib aus klinischer Sicht erstrangig

B Ila, Ilb, III aus klinischer Sicht zweitrangig

C IV aus klinischer Sicht drittrangig

D $\quad$ V aus klinischer Sicht viertrangig

\subsubsection{Evidenzbasierte Medizin und Rhinoplastik}

Wie schon in den vorangegangenen Kapiteln beschrieben, fehlen uns in der Breite hochwertige kontrollierte randomisierte Studien, selbst bei der reinen Septumchirurgie. Es existieren nur einzelne Arbeiten zu singulären Vorgehensweisen in der Rhinochirurgie [57]. Multiple Metaanalysen zu verschiedenen Bereichen der Rhinoplastik wurden publiziert (siehe Teil 2), die sich allerdings auf Einzelstudien oder Einzeloperateursergebnissen ohne Randomisierung und Kontrolle stützen. Es sollte Ansporn in der Rhinoplastik, wie in allen operativen Fächern sein, sich dieser Herausforderung zu stellen. Zunächst müsste dies national organisiert und die Ergebnisse verglichen werden, um dann den nächsten Schritt im internationalen Austausch zu gehen. Angesichts der enormen positiven Entwicklung inklusive der etablierten S2k Leitlinien, die die plastisch-ästhetische Nasenchirurgie zum Wohle ihrer Patienten in den letzten Jahren im Bereich der OP-Techniken genommen hat, können wir optimistisch dieser Aufgabe entgegensehen. Darüberhinaus sei erwähnt, dass es schon EbM Anwendungen in der Inneren Medizin, Chirurgie, Psychiatrie und Allgemeinmedizin seit Mitte der 1990er Jahre gibt [5860]. Es sei in diesem Zusammenhang nur daran erinnert, wie sich z. B. die Therapie des Larynxkarzinoms in den letzten 20 Jahren, aufgrund Evidenzbasierter Daten, komplett verändert hat [61].

\section{Schlussfolgerung zum Teil 1}

Aus Sicht aller drei Akteure (Arzt, Patient, Kostenträger) muss Qualität im Gesundheitswesen transparent, dauerhaft, wissenschaftlich nachvollziehbar und kostengünstig sein. Der Fokus liegt zum Teil in unterschiedlichen Bereichen. Der Arzt möchte die beste Versorgung für seinen Patienten, die er mit seinem Können und Wissen kombinieren kann. Der Patient möchte die beste Qualität mit den geringsten Nebenwirkungen, dem schönsten Ergebnis und zumindest im Selbstzahlerbereich zu überschaubaren Kosten. Die Kostenträger streben eine Qualität an, die primär den Kostenrahmen nicht sprengt und mit Wirtschaftlichkeit und Einsparungen zu vereinbaren ist. Dies ist ein Spagat, der kaum zu bewerkstelligen ist, ohne dass der eine oder andere Teil Schaden nimmt oder unterrepräsentiert ist. Um dies einvernehmlich und mit harten Daten zu untermauern, sollte die Ausarbeitung von Evidenzbasierten Daten auch für die plastisch-ästhetische Nasenchirurgie angestrebt werden. Allerdings müssen sie frei von monetären Einflüssen, alten Gewohnheiten oder unrealistischen Erwartungen die reinen Daten und Fakten darstellen und interpretieren. 


\section{Teil 2 - Qualität von Operationsmethoden in der Rhinoplastik}

Die im ersten Teil gewonnen Erkenntnisse gilt es nun auf den Bereich der rhinochirurgischen Operation auszudehnen. Hier spielen, neben den für alle Bereiche der Medizin geltenden Normen, besonders im Bereich der Veränderung der äußeren Form die emotionalen und weichen Kriterien im Empfinden der Patienten eine wesentliche Rolle.

Die plastisch-ästhetische Nasenchirurgie scheint sich immer epochenweise zu entwickeln. Dies geschieht meistens durch wegweisende Publikationen, die durch neue OP-Techniken begleitet werden. Ein Wendepunkt in den letzten 40 Jahren war das von Sheen 1978 veröffentlichte Werk „Aesthetic Rhinoplasty“ [62]. Sheen läutete einen ersten großen Paradigmenwechsel seit Jacques Josephs Buch aus dem Jahre 1931 ein [63], denn die Rhinoplastik etablierte sich zu einer richtigen ästhetischen Operation mit präoperativer Analyse, operativer Planung und chirurgischer Durchführung. Das Reduktionskonzept von Joseph wurde durch eine ausgewogene Operation mit der Kombination aus Resektion und Transplantaten ersetzt und die z. T. unschönen Ergebnisse, die zu Revisionen führten, konnten deutlich verbessert werden. Dadurch wurden Rhinochirurgen nicht mehr über deren Schnelligkeit, sondern über ihre ästhetischen Ergebnisse definiert.

Die klassischen Fächer, wie Physiologie und Anatomie, von denen häufig gedacht wurde „alles sei schon verifiziert und untersucht“ überraschen bis heute mit weiteren Details, sodass neue Erkenntnisse unser Verständnis von Form und Funktion der Nase verbessern und die operative Vorgehensweise stetig verändern und verfeinern. Insgesamt hat sich die Art der Nasenoperation in den letzten Jahrzenten enorm weiterentwickelt und präzisiert.

Große Fortschritte wurden durch den erkannten Zusammenhang zwischen Anatomie, nasaler Ästhetik und chirurgischer Technik herausgearbeitet. Als Beispiel seien die Nasenspitze mit ihren nasalen Bändern und das osteokartilaginäre Nasengewölbe genannt.

Als die offenen Nahttechniken für die Nasenspitze in aller Munde waren, gelang es Cakir darzulegen, dass ähnliche Ergebnisse an der Nasenspitze mit besserer Kontrolle und weniger Morbidität durch einen geschlossenen Zugang zu erreichen waren [64]. Ziel war es die nasalen Bänder zu erhalten und die Knorpel nur minmal zu resezieren. Dies gelang durch einen subperichondralen Zugang mit geringerer postoperativer Schwellung und reduziertem Taubheitsgefühl, sowie weniger Narbenbildung, was auch für die Zukunft eine mögliche Revision erleichtern würde.

Ein weiterer Wendepunkt in der Philosophie der Nasenspitzenchirurgie waren die Arbeiten von Ozmen [65] und Gruber [66], die das Sliding bzw. den Erhalt der Flügelknorpel postulierten, da es bis dahin üblich war, den cephalen Anteil grundsätzlich zu resezieren. Aufgrund dieser Neuerungen waren sowohl das sogenannte „alar notching“ postoperativ weniger zu sehen als auch die Verwendung von Alar rim grafts deutlich seltener. Als weiteres Beispiel sei die Problematik der „alar malposition“ erwähnt, die als eine der schwierigsten Deformitäten galt und mit „alar transpositioning“ und „lateral crural strut grafts“ zu therapieren war. Davis konnte überzeugend darstellen, dass dem mit dem „medial tensioning“ ausreichend begegnet werden konnte, ohne dass eine Transposi- tion bzw. eine alare Resektion durchgeführt oder zusätzliche Grafts verwendet werden mussten [67].

Am osteokartilaginären Nasengewölbe konnten anatomische Studien zeigen, dass der knöcherne Höcker in Wahrheit nur eine kleine Knochenkappe ist, die man einfach entfernen und dabei das darunterliegende knorpelige Gewölbe erhalten kann [68].

Der immer im Fokus stehende Keystonebereich hat sich als eine Art semimobiles knorpeliges Gelenk herausgestellt, das von der konvexen in die gerade Form durch Resektion der knorpeligen Septumabstützung verändert werden kann [69].

Im Fokus der operativen Qualität möchten wir 4 Bereiche beleuchten, die im öffentlichen Diskurs auf Kongressen und Symposien seit Jahren diskutiert und die je nach Interpretationssicht als qualitätsstiftend angesehen werden.

- Offen vs. Geschlossener Zugang

- Rippen-vs. Ohrknorpel

- Piezoosteotomie vs. traditionelle Osteotomie

- Preservationsrhinoplastik vs. Non-preservation

\section{Offenen vs. geschlossenen Zugang}

Beim Blick in die Historie zeigt sich, dass schon in der Zeit vor dem zweiten Weltkrieg offene und geschlossene Zugänge praktiziert wurden. Ende des 19. Jahrhunderts führte man die Operationen offen durch, da noch keine Möglichkeiten seitens des Lichts und der Instrumente bestanden, diese Eingriffe geschlossen durchzuführen. Erst mit der technischen Weiterentwicklung der OP-Beleuchtung und der Stirnlampen nach dem zweiten Weltkrieg etablierte sich zunehmend die geschlossene Technik, da man äußerlich keine Narben sah und sie somit unter den Chirurgen als eleganter empfunden wurde. Eigentlich fast alle Nasenchirurgen, die ab den 1960er Jahren die funktionelle und ästhetische Rhinoplastik in Europa erlernten, haben dies durch einen geschlossenen Zugang getan. Die Einführung der Endoskopchirurgie hat der geschlossenen Technik zusätzlich einen Vorschub geleistet.

Die offene Technik verlor sich nach dem zweiten Weltkrieg zunehmend in Europa und wurde Stück für Stück in den USA weiterentwickelt. Als der geschlossene Zugang an seinem Höhepunkt in der Betrachtungsweise angelangt war, begann wieder ein entscheidender Richtungswechsel durch die Arbeiten von Goodman Anfang der 1970er Jahre [70]. Die Ergebnisse überzeugten auch andere Rhinochiurgen in den USA, sodass die Technik sich sehr schnell in den Publikationen verbreitete [71-73].

In den 1990er Jahren kam, die im 19. Jahrhundert schon in Deutschland durch Diefenbach und Joseph entwickelte und etablierte offene Technik wieder zurück nach Europa und erreichte ihren Siegeszug auch im Bereich der ästhetischen Rhinoplastik.

Diese chirurgische Revolution fusste auf drei Säulen:

1. Der offene Zugang ermöglichte eine bessere Visualisierung für die Analyse, OP-Technik und Lehre

2. Es entwickelten sich neue OP-Techniken, wie Nasenspitzennähte, Rekonstruktion des mittleren Gewölbes oder des Septums, die beim geschlossenen Zugang unmöglich oder sehr schwierig waren

3. Der offene Zugang reduzierte die Lernkurve für den Anfänger, wodurch die Rhinoplastik zu einer der am meisten durchgeführten ästhetischen Operationen wurde 
Aiach schrieb allerdings noch 1992, dass die offene Chirurgie nur eine Option bei Revisionen, schwierigen Fällen oder der Nasenspitzenchirurgie sein könne und keineswegs zur Standardprozedur werden sollte [74]. Allerdings hat sich dies mittlerweile aus heutiger Sicht komplett überholt, da über $70 \%$ aller Rhinoplastiken in offener Technik praktiziert werden. Natürlich gab es auch Stimmen, dass die offene Technik eine Rückentwicklung der Chirurgie in das 19. Jahrhundert sei und bezeichneten den offenen Zugang als „chirurgische Todsünde“ [75]. Diese Stimmen verstummten aber aus den zuvor genannten Gründen recht schnell wieder.

Eine wissenschaftliche Qualitätsanalyse gab es in der heute geforderten Art damals für beide Zugänge nicht, denn die Techniken wurden auf Kongressen vorgestellt, von erfahrenen Operateuren Ihrer Zeit postuliert und in den Kliniken entsprechend im Rahmen der Weiterbildung etabliert.

Die Qualität wurde insbesondere unter dem Aspekt der Narbe an der Columella diskutiert und ob die äußerlichen Ergebnisse mit der einen oder anderen Technik besser oder schlechter seien [76]. Eine Diskussion hinsichtlich Qualität bei Patientenkomfort, Atmung, Funktion, Riechvermögen, Lebensqualität usw. hat dahingehend erst viel später stattgefunden. Lediglich in der Einschätzung, dass Revisionen, Traumanasen bzw. Spaltnasen durch die offene Technik besser zu analysieren und zu operieren seien, gab es schlussendlich einen Konsens (s. o.).

\subsubsection{Qualität der äußeren bzw. ästhetischen Ergebnisse} In der „Renaissance“ des offenen Zugangs postulierten schon 1988 Friedman und Gruber, dass die Langzeitergebnisse besser und dauerhafter seien. Aufgrund der offenen Darstellung der Anatomie seien insbesondere Präzision, Genauigkeit und die Vorhersagbarkeit des Ergebnisses dem geschlossenen Zugang überlegen. In der Analyse lag der Fokus primär auf dem Nasengrüst und der Nasenspitze. Das Argument der Narbe am Nasensteg war für Gruber damals schon nicht mehr von Belang, da verbesserte Nahttechniken eine deutliche Verbesserung der postoperativen Situation zeigten [77].

In einer neuen Studie aus dem Jahre 2017 konnte die Gruppe um Yagmur und Demir an 91 Patienten zeigen, dass die Columellanarbe keinerlei signifikanten Einfluss auf die Zufriedenheit des Patienten gegenüber der Rhinoplastik im speziellen und auch in der Körperwahrnehmung im allgemeinen hat [78].

Nichtsdestotrotz darf man mit beiden Techniken die Lernkurve und die damit bestehenden Risiken und Nebenwirkungen nicht außer Acht lassen. Bei geschlossenem wie offenem Zugang besteht die Gefahr des überresezierten Nasenrückens bzw. der Nasenspitze sowie das Entstehen einer engen inneren Nasenklappe. Darüberhinaus sind beim offenen Zugang zusätzliche Risiken einer engen oder instabilen äußeren Nasenklappe, einer instabilen Nasenspitze sowie der Kurznase beschrieben. Darüberhinaus ist es bemerkenswert, dass die Anzahl der Patienten mit Mehrfachoperationen der Nase bei einem offenen Zugang deutlich höher, als bei einem geschlossenen Zugang ist [79].

In der Re-Etablierungsphase, in der auch nasenchiurgische Anfänger den offenen Zugang ohne Berührungsängste erlernten, brachte die ungeübte Gewebepräparation im Nasenspitzenbereich ungekannte Traumatisierungen und Durchblutungsstörung mit sich, welche die Qualität des offenen Zugangs zu Anfang schmäler- ten. Der Vorteil der offenen Darstellung der Nasenspitze und des Nasenrückens brachten dadurch gleichzeitig gravierende Nachteile mit sich. Anatomische Strukturen traten wieder in den Vordergrund, die bei den Operateuren in Vergessenheit geraten waren, da der geschlossene Zugang diese nicht primär tangierte. Die nasalen Bänder wurden trotz ihres Einflusses auf Funktion und Ästhetik lange Zeit übersehen [80].

Als Beispiel seien das „Pitanguy Ligament“ genannt, das schon 1965 durch Pitanguy dargestellt und dessen Rolle bei der dicken postoperativen Nasenspitze beschrieben wurde [81]. Des Weiteren das vertikale Scroll Ligament, das die innere Nasenklappe durch den Muskulus transversalis nasi stabilisiert und die Funktion des Nasengewölbes unterstützt [68].

Darüberhinaus konnte Toriumi 1996 in einer sehr schönen Kombinationsarbeit aus klinischer Evaluation, anatomischer Dissektion und histologischer Aufarbeitung den Verlauf von Venen, Arterien und Lymphgefäßen im Bereich der Nase darstellen [82]. Arterien, Venen und Lymphgefäße verlaufen in oder über der muskuloaponeurotischen Gewebeebene der Nase. Somit wurde das Risiko der ausgeprägten Nasenspitzenschwellung oder sogar der Hautnekrose in diesem Bereich durch eine strikte Präparation unterhalb der muskuloaponeurotischen Schicht deutlich reduziert und somit natürlich auch die Qualität entsprechend verbessert.

\subsubsection{Qualität der funktionalen bzw. endonasalen Ergebnisse}

Im Bereich der Funktion und der knorpeligen und knöchernen Architektur kommt es auf den Schweregrad der Veränderung an. Einfachere Deviationen, Sporne usw. können problemlos mit einer geschlossenen modernen Septorhinoplastik korrigiert werden, bei denen ein offener Zugang völlig übertherapiert wäre. Bei größeren Veränderungen im Inneren der Nase, die eine extrakorporale Rekonstruktion bzw. Wiederherstellung des knorpeligen bzw. knöchernen Nasengerüsts erfordern, stößt man mit der geschlossenen Technik an Grenzen, sodass der offene Zugang im allgemeinen als Standard anerkannt wird. Berghaus beschrieb allerdings in seinem Artikel 2016 ausführlich, dass auch in der modernen Rhinoplastik noch genügend Raum für einen geschlossenen Zugang gebe, der zusätzliche Vorteile bietet [83]. Insbesondere in der Rekonstruktion des Septums, der inneren und äußeren Nasenklappe, der vorderen Abstützung und der Nasenspitze ist der offene Zugang, auch durch seinen großen Anteil an verwendeten Knorpeltransplantaten (grafts) dem geschlossenen Zugang überlegen.

Qualitätsvergleichsstudien sind bezüglich der reinen funktionellen Ergebnisse nicht bekannt. Allerdings hat sich die Überlegenheit des offenen Zuganges in der Rekonstruktions- und Revisionschirurgie im Laufe der Jahre gezeigt und ist entsprechend anerkannt (s. o.).

\subsubsection{Qualität der subjektiven Patienteneinschätzung}

Interessanterweise gibt es seitens der subjektiven Einschätzung der Patienten hinsichtlich des Zugangs, trotz jahrelanger Fachdiskussionen, die bis heute $z$. T. andauern, keine signifikanten Abweichungen. Verschiedene Scores und Fragebögen (NOSE, ROE, DAS24) zeigen keinen signifikanten Unterschied. In einer aktuellen Arbeit aus dem Jahre 2019 konnten Gökce Kütük und Ok an über 90 Patienten zeigen, dass der psychosoziale Stresslevel der Patienten nach einer erfolgreichen Rhinoplastik deutlich reduziert wurde 
[84]. Dieser Wert war aber völlig unabhängig von der Art des Zugangs (offen vs. geschlossen), der Indikation (ästhetisch vs. funktionell) oder des Eingriffstyps (primär vs. Revision). Dies zeigt, wie auch andere prospektive Follow-up-Studien in diesem Bereich, dass die Art des Zugangs nicht entscheidend ist, sondern das Ergebnis speziell in der Betrachtung des Patienten [85]. Die wiederkehrende Bedeutung der Ergebnisqualität vs. Prozedurqualität wird hierbei sehr deutlich.

\section{Rippen- vs. Ohrknorpeltranplantat}

Ein wesentlicher Pfeiler der traditionellen Rhinoplastik war die dorsale Resektion mit Zerstörung des Keystonebereichs und sofortiger Rekonstruktion mittels Osteotomien und Gewölberekonstruktion. Trotz aller operativer Verbesserungen im Bereich der Ästhetik und Funktion der Nase, bleiben bis heute kleinere Revisionen oder große Zweit- und Dritteingriffe unvermeidlich. Jenseits der Traumaanamnese sind es die Revisionen, bei denen die Verwendung von Rippen- bzw. Ohrknorpel zur Rekonstruktion des Nasenrückens oder der Nasenspitze eine Rolle spielen. Die Technik der Preservation im Bereich des Nasenrückens könnte die Anzahl der Nasenrückenrevisionen mit Rippen- oder Ohrknorpel vermeintlich reduzieren und die Notwendigkeit einer Gewölberekonstruktion minimieren (s. Kapitel 11) [86].

In der Analyse der unzähligen Verbesserungen muss man sich in der Historie allerdings die Frage stellen, die Rollin Daniel einmal so treffend formuliert hat: „Warum führen wir eine Operation durch, die solch ein zerstörerisches Ergebnis produzieren kann, dass Rippen- oder Ohrknorpel im Rahmen der Revision benötigt wird, auch wenn der Ersteingriff von einem erfahrenen Operateur durchgeführt wurde?"[87].

Der Einsatz von Knorpeltransplantaten zum Beispiel aus der Rippe, mit primärer Rekonstruktion der Basis und darauffolgendem Aufbau der sekundären Strukturen ist heute unbestritten. Formationen, die sich langfristig den Kontrakturkräften widersetzen sind hierbei von entscheidender Bedeutung [88].

Um eine genauere Betrachtung zu ermöglichen, ist eine Aufteilung in Rippen- und Ohrknorpeltransplantate nötig. Bei Verwendung der Rippe die zusätzliche Unterteilung in autologen bzw. bestrahlten homologen Knorpel. Des Weiteren muss man den operativen Entwicklungen Rechnung tragen und mittlerweile auch die jeweils gewürfelte Form mit (Diced cartilage in fascia, DCF) und ohne Faszie (free diced cartilage, FDC) berücksichtigen. Da die komplette Aufarbeitung der Knorpeltransplantate für die gesamte Nasenarchitektur den Rahmen des Referats sprengen würde, wurde der Fokus auf den Nasenrücken gelegt.

\subsubsection{Qualität der äußeren bzw. ästhetischen Ergebnisse}

\subsubsection{Autologer Rippenknorpel (ACC)}

Der autologe Rippenknorpel ist eine der ältesten Formen in der Rekonstruktion des Nasenrückens. Schon Ende der 1960er Jahre [89] und Mitte der 1970er Jahre wurden Arbeiten hierüber publiziert [90]. Mittlerweile hat sich die Technik etabliert und verfeinert. In der Verwendung des Rippenspans zur Augmentation des Nasenrückens liegen mittlerweile sehr lange Beobachtungszeiträume vor, die eine gute Übersicht über die äußeren Ergebnisse liefern. Eine Metaana- lyse aus über 10 Studien, fast 500 Patienten und einem mittleren Nachbeobachtungszeitraum von fast 3 Jahren zeigte, dass die Beschwerden im Bereich Verkrümmung, hypertrophe Narbe und Revision lagen [91]. Dies ist insbesondere beachtenswert, da diese drei Punkte auch von Patienten und deren Umgebung wahrgenommen werden. Die Verkrümmung (3,1\%) des eingelegten Rippenspans bedeutet für Patienten häufig eine Enttäuschung. Die hypertrophe Narbe (5,5\%) am Korpus ist ein Stigma und eine ästhetisch deutlich einschränkende Folge des Hebeeingriffs, insbesondere bei Frauen. Andere Komplikationen, wie Resorption, Infektion, Verrutschen des Rippenspans usw. spielten kaum eine Rolle und lagen unter $0,6 \%$. Eine Revisionsrate von $14 \%$, jeder 7. Patient(!), lies somit auch die Frage aufkommen, ob es nicht noch andere Möglichkeiten gäbe, auf die wir weiter unten zu sprechen kommen.

\subsubsection{Bestrahlter homologer Rippenknorpel (IHCC)}

Die erste publizierte Arbeit über IHCC wurde 1961 veröffentlicht. Hierbei beleuchtete die Gruppe, um Dingman die Möglichkeit homologen Rippenknorpel mittels Bestrahlung haltbar zu machen [92].

Kridel berichtete 1993 schon an über 120 Nasenrückenaugmentationen über eine gute Verwendungsmöglichkeit des bestrahlten homologen Rippenknorpels [93]. Auch in den Publikationen der Verlaufsergebnisse aus den Jahren 2009 [94] und 2017 [95] konnte Kridel bestätigen, dass die Gesamtsumme an Nebenwirkungen an 943 Patienten nur 3,25\% war. Hierbei machten die Bereiche Resorption 1,01\% und Verkrümmung 1,06\% den größten Bereich aus. Insgesamt zieht Kridel ein rundum positives Fazit, da die Nebenwirkungsraten sehr gering sind, die Hebemorbiditäten komplett entfallen und der IHCC kostengünstiger als der ACC sei.

In einer großen retrospektiven Vergleichsstudie inklusive histologischer Aufbereitung zwischen ACC und IHCC, ebenfalls aus 2017 [96], wurden 83 Patienten nachuntersucht. Es kamen Rippenspäne mit einem Ausmaß von 30 bis 40 mm Länge, 4 bis 6 mm Dicke, and 8 bis $10 \mathrm{~mm}$ Breite zum Einsatz. Es konnte eine 10-mal höhere Resorptionsrate (30\%) beim IHCC nachgewiesen werden. Dies spiegelte sich natürlich auch im optischen Resultat wider, das dem ACC signifikant unterlegen war. Interessanterweise drücken diese Zahlen auch die allgemeine Literatur im Gegensatz zu Kridels Arbeiten aus, bei der die Resorptionsrate je nach Follow-up beim IHCC zwischen 1,4 und 75\% liegt. Die Unterschiede bei Verkrümmung oder Infektion waren nicht signifikant. Auch im Funktionsbereich gab es keine objektiven Unterschiede.

\subsubsection{Qualität der subjektiven Patienteneinschätzung zwi- schen ACC und IHCC}

Die o.g. Ergebnisse projizieren sich natürlich auch auf das subjektive Qualitätsempfinden der Patienten. In Kridels Arbeit 2009 [94] verbesserte sich im mittleren Nachbeobachtungszeitraum von 7,8 Jahren die hohe subjektive Patientenbewertung beim inneren wie äußeren Ergebnis von 91,3 auf 94,2\%.

In der Studie von Wee et al. wurde die postoperative Zufriedenheit in beiden Gruppen mit „zufrieden“ oder „sehr zufrieden“ bei über $75 \%$ der Patienten angegeben [96]. Allerdings war, aufgrund der hohen Resorptionsrate des IHCC, der intergruppale Wert im ästhetischen Bereich signifikant schlechter. Einen signifikanten inter- 
gruppalen Unterschied im funktionalen Anteil konnte nicht nachwiesen werden. Hier lag die Zufriedenheit, wie bei Kridel bei $95 \%$. Hier lässt sich wieder sehr gut darstellen, dass die Patientenzufriedenheit primär vom Ergebnis und nicht von der Methode selbst abhängt.

\subsubsection{Gewürfelter Knorpel in Faszie (Diced Cartilage in Fascia)} Der gewürfelte Knorpel wurde von Erol als sogenanntes „Turkish Delight“ für den Bereich der Nase nach 10-jähriger Anwendung erstmalig publiziert [97]. Er wickelte den Knorpel in seinen Publikationen in Surgicel ${ }^{\circledR}$ und nicht in Faszie ein. Daniel und Calvert konnten in ihrer Arbeit aus dem Jahre 2004 auch klinische Schwierigkeiten darlegen und sahen den Grund in einer Fremdkörperreaktion des bis dato verwendeten Surgice ${ }^{\circledR}[98]$. Nach 2006 etablierte sich die Verwendung von Faszie, da Calvert histologisch die Überlegenheit von gewürfeltem Knorpel in Faszie gegenüber dem Surgicel nachweisen konnte [99]. In neueren Arbeiten wird auch mit Cellulose anstatt Faszie experimentiert, wobei die Anwendung am Menschen noch aussteht [100].

In der Langzeitbetrachtung kam Erol nach 25 Jahren, allerdings mit Surgicel ${ }^{\circledR}$, zum Schluss, dass dies eine optimale Methode ohne entsprechende Nebenwirkungen sei obwohl sich mittlerweile die weiter verbreitete Faszienanwendung etabliert hat [101]. In einer Weiterentwicklung hat Tasman die Faszie durch Fibrinkleber bzw. Blutbestandteile zur Stabilisierung der Würfel ersetzt und konnte ebenfalls bei über 100 Patienten zufriedenstellende Ergebnisse nachweisen [102]. Für alle Weiterentwicklungen des klassischen Rippenspans gilt, dass die Patienten die Verwendung von Diced Cartilage, unabhängig von der Wickelform, als angenehmer gegenüber dem festen Rippenspan empfunden haben. Dies ist der o.g. verminderten Rigidität und Verkrümmungstendenz geschuldet [103].

\subsubsection{Faszienfreier loser gewürfelter Knorpel (Free Diced Car- tilage)}

Hieraus leitete die Gruppe um Gubisch eine Weiterentwicklung ab, die 2017 als FDC publiziert wurde [104]. Hierbei verglichen Sie sowohl für die Augmentation als auch die Camouflage den Einsatz von FDC entweder ohne jegliche Wickelkomponente inklusive Fibrin oder mit Faszie oder als klassisches DCF. Interessanterweise war die Revisionsrate in der DCF Gruppe fünfmal höher als in der FDC Gruppe. Dies mag sicherlich auch daran gelegen haben, dass FDC viel kleiner und feiner gewürfelt werden muss, fast wie Puderzucker, als im Gebrauch von DCF. FDC ist eine gute Option insbesondere für das Abdecken von Irregularitäten und den finalen Touch up. Patienteneinschätzungen liegen hierzu aufgrund der Kürze des Gebrauchs noch nicht vor.

\subsubsection{Ohr- bzw. Conchaknorpel}

Münker machte schon 1984 deutlich, dass in der Verwendung des Ohrknorpels als doppelten oder dreifachen Sandwich eine Option zum Rippenspan für die Augmentation des Nasenrückens besteht [105].

Hierbei gab es aber die entsprechenden Probleme der Knorpelluxation mit unschönen äußerlichen Ergebnissen. Darüberhinaus war, aufgrund der reduzierten Spendermenge, nur ein begrenzter
Nasenrückenaufbau möglich. Somit war auch beim Ohrknorpel der Schritt zum gewürfelten Knorpel, nachdem auch tierexperimentell bewiesen werden konnte, dass insbesondere unter Beibehaltung des Perichondriums eine bessere Vaskularisierung und Stabilisierung des Transplantates erzielt werden konnte, vorgezeichnet [106]. Auch beim Ohrknorpel gibt es eine Hebemorbidität, die sich hauptsächlich durch Keloide oder Hämatome auszeichnet. Sie liegt nach aktuellen Studien bei ca. 2,4\% [107]. Einen Qualitätsunterschied in der Knorpelart (Rippe vs. Ohr) oder Herkunft der Faszie (autogen vs. allogen) konnte im Tierexperiment nicht nachgewiesen werden. Kleinere Unterschiede waren nur in Ossifikation und Kalzifikation ohne Anhalt auf das Ergebnis zu verzeichnen [108].

\subsubsection{Qualität der subjektiven Patienteneinschätzung zwischen Rippen- und Ohrknorpel}

Seit der Etablierung der „diced cartilage“ Technik, egal in welcher Form, hat die Patientenzufriedenheit und Qualitätseinschätzung deutlich zugenommen, da die Verkrümmungstendenz entfallen ist. Auch die Rigidität wird seitens der Patienten nicht mehr als störend angegeben, da die anderen Formen deutlich weicher in der Haptik sind [103]. Hinsichtlich der Hebemorbidität kommt es sicherlich auch auf die Erfahrung des Operateurs an. Neueste Untersuchungen zeigen, dass Patienten keinen Unterschied zwischen Ohr- oder Rippenknorpelentnahme empfinden [109], was wieder zur Ergebnisqualität führt.

\section{Ultraschallbasierte vs. konventionelle Osteotomie}

Die konventionelle Osteotomie hat ebenfalls im Laufe der Jahrzente eine Wandlung insbesondere durch den Wechsel vom geschlossenen zum offenen Zugang erfahren. Ursprünglich mit dem gekehlten Waltermeisel endonasal durchgeführt, entwickelte sich die Technik zur externen lateralen, transversalen und paramedianen Osteotomie, die erstmals durch Goria 1955 beschrieben und durch Straatsma 1961 wissenschaftlich publiziert wurde $[110,111]$.

Ihre klinische Überlegenheit bei Schwellung, Blutung, Einblutung und Mukosaverletzung konnte in vielen Studien sowohl mit durchgehender bzw. perforierender Frakturlinie - im Sinne einer Grünholzfraktur - auch am Kadaver gezeigt werden [112, 113]. Prinzipiell waren diese Studien darauf fokussiert das Handling und die Kurzzeitergebnisse zu beschreiben, aber nicht die mittel- und langfristige Verbesserung der postoperativen Lebensqualität der Patienten.

Durch den technischen Fortschritt haben andere Methoden Einzug gehalten. Die Osteotomie mit Ultraschall ist in anderen Fachbereichen, wie z. B. der Zahnmedizin, schon viel länger als in der Nasenchirurgie etabliert. Robiony und seine Arbeitsgruppe beschrieben die Anwendung der Piezochirurgie in der Rhinochirurgie erstmals 2007 [114]. Sie haben hierbei mittlerweile weltweit die längste Erfahrung, allerdings in der perkutanen Anwendung. Ihr 10-jähriges Follow-up, welches 2019 publiziert wurde, beschreibt an 183 Patienten die Piezoosteotomie mit keiner oder milder Schwellung bei über $90 \%$ der Patienten. Die weitere Entwicklung geht mittlerweile hin zum piezonavigierten Zugang, der allerdings noch nicht etabliert ist [115]. Im Gegensatz hierzu führt die Grup- 
pe um Gerbault und Daniel die Ultraschall-osteotomie durch einen erweiterten offenen Zugang mit weit aufpräpariertem Weichteilmantel durch [116]. Berghaus kam in einer 2019 veröffentlichten retrospektiven Studie an 35 Patienten zu dem Schluss, dass die Piezotechnik in der Rhinoplastik bei der Knochenmodellierung und Osteotomie eine sichere und präzise Vorgehensweise ermöglicht [117].

Die Arbeitsgruppe um Kurt Yazar konnte an einem Tiermodell im Vergleich zeigen, dass es durch die Piezotechnik im Gegensatz zur perkutanen bzw. endonasalen Osteotomie zu weniger Schleimhautläsionen und unerwünschten Bruchlinien kommt [118]. Dies entspricht auch den bisher beschrieben Erfahrungen der regelmäßigen Anwender dieser Osteotomietechnik. Langzeitergebnisse liegen aber in allen durchgeführten Studien noch nicht vor. In den bisher dargestellten Daten zeigt sich zumindest kein offensichtlicher Nachteil dieser Technik, allerdings lies sich aber bisher auch kein signifikanter Vorteil postulieren. Hinsichtlich der Lebensqualität der Patienten gibt es bisher keinerlei Erhebungen, die einen Vergleich zuließen.

\subsubsection{Qualität der äußeren bzw. ästhetischen Ergebnisse} Kocak et al. beschrieben 2017 in einer prospektiven Studie an 49 Patienten, dass insbesondere die Schwellung in den ersten beiden Tagen postoperativ bei der Piezoosteotomie signifikant geringer war, als bei der konventionellen Osteotomie [119]. Diese Signifikanz verlor sich sehr schnell bis spätestens zum siebten postoperativen Tag wieder. Da die reduzierte Schwellung nur in den ersten 48 Stunden bei beiden Piezomethoden (offen vs. perkutan) beschrieben ist, kann dieser Effekt nicht am Umfang der Weichteilpräparation liegen. Bei der Ekchymose ist die Piezochirurgie im kompletten Nachbeobachtungszeitraum der konventionellen Osteotomie signifikant überlegen. Dies konnte auch in einer systemischen Literaturanalyse durch Währmann et al. 2019 bestätigt werden [120]. Ob die begleitend geringer beschriebenen Schmerzen wirklich signifikant reduziert waren oder nur eine Folge des geringeren Ekchymoseeindrucks der Patienten ist, bleibt bisher im Verborgenen. Zu Recht beschreiben die Autoren diese Ergebnisse als Trend, da sie noch nicht statistisch verifiziert werden können und Langzeitergebnisse in allen Bereichen völlig fehlen.

\subsubsection{Qualität der funktionalen bzw. endonasalen Ergebnisse} Wissenschaftliche Arbeiten über die funktionellen zw. endonasalen Ergebnisse liegen auch nach über 10 Jahren Follow-up leider nicht vor. Dies wäre aber insbesondere angesichts der beschriebenen geringeren Schleimhautläsionen und Mukosa-schädigungen zu wünschen. Entsprechende Untersuchungen sind auch bis heute im Vergleich endonasaler zu perkutaner Osteotomie nicht gemacht worden, obwohl ebenfalls die Überlegenheit der externen Osteotomietechnik damals im Bereich der Mukosaverletzung beschrieben wurde.

\subsubsection{Qualität der subjektiven Patienteneinschätzung}

Valide Studien sind leider bisher nicht durchgeführt worden. Lediglich die signifikant verringerte Schwellung im kurzen postoperativen Intervall (bis 2 Tage postoperativ) und die geringere Ekchymose wurden seitens der Untersucher verifiziert und beschrieben (s. o.). Hinsichtlich der Lebensqualität oder Funktionseinschrän- kung gibt es keine validen Daten und Studien, die sicherlich folgen werden. Allerdings konnten Baumann et al. nachweisen, dass es keinen Unterschied in der subjektiven Patienteneinschätzung bzw. der Lebensqualität hinsichtlich der Osteotomie von innen oder von außen gibt [85]. Lediglich die o.g. geringeren Schmerzangaben der Patienten bei der Piezoosteotomie zeigen einen subjektiven Unterschied in den verschiedenen Osteotomietechniken.

\section{Preservation vs. traditionelle Rhinoplastik}

Die Preservationsrhinoplastik ist im Moment in aller Munde. Sie ist keine neue Herangehensweise im Bereich der Rhinoplastik. Erstmal wurde diese Technik 1899 von J.L. Goodale im Boston Medical Surgical Journal unter dem Titel „A new method for the operative correction of exaggerated roman nose “ für den Bereich des Nasenrückens veröffentlicht [121]. Obwohl damals schon der Erhalt des Keystoneareals postuliert wurde, verlor sich dieses Wissen im Laufe der Zeit, wie oft in der Medizin, insbesondere nachdem Joseph 1931 [63] die Reduktionstechniken mit Abtragen des Höckers im Keystonebereich postulierte.

Es zeigt sich an der Wiederentdeckung der Preservation, dass sich im Bewußtsein des Anatomieerhalts anscheinend wieder eine neue/alte Herangehensweise Bahn bricht. Seit Sheens Buch aus dem Jahre 1978 (s. o.) hat sich die Rhinoplastik vom Resezieren zum Umgestalten entwickelt (resection to reshaping). Jetzt scheint die Entwicklung von der Umgestaltung zur Aufrechterhaltung der anatomischen Strukturen zu gehen (reshaping to preservation). Dies ist insbesondere durch die neu gewonnenen Erkenntnisse aus der Anatomie und Physiologie (s. o.), verbesserten Nahttechniken und verfeinerten OP-Techniken möglich.

Die ersten beschriebenen Ergebnisse einer sogenannten „push down“ Preservationsrhinoplastik gab es 1975 durch Barelli [122]. Yves Saban entdeckte diese Technik wieder und entwickelte sie im Rahmen eines geschlossenen Zugangs weiter [123]. Prinzipiell wird die Technik heute als komplette Erhaltung aller Strukturen oder partielle Erhaltung bestimmter Strukturen (Haut/Nasenrücken, Haut/Nasenspitze, Nasenspitze/Nasenrücken) charakterisiert. Yves Saban publizierte 2018 mit seiner Gruppe eine Studie an $320 \mathrm{~Pa}$ tienten und unterschied in let-down-Technik (LDT) und push-downTechnik (PDT) [86].

Prinzipiell besteht der Unterschied darin, dass die PDT bei kleineren Höckern bis zu 4mm indiziert ist und die voll mobilisierte Nasenpyramide „runtergedrückt“ wird. Die Erkenntnis und Bedeutung des Keystonebereichs als semimobiles kartilaginäres Gelenk wurde schon zuvor beleuchtet [69]. Bei der LDT wird nach einer zusätzlichen Keilexcizion im Bereich der Maxillafortsätze die Nasenpyramide „heruntergelassen“ und ist für Höcker über $4 \mathrm{~mm}$ indiziert. Die Preservationstechnik ist nicht ausschließlich eine Technik des geschlossenen Zugangs, sondern wird auch bei offenen Zugängen praktiziert. Rollin Daniel publizierte 2019 einhundert ausschließlich offen operierte Fälle mit einem Follow-up von einem Jahr [124]. Er sieht die Technik als erneuten Paradigmenwechsel an, die den dorsalen Hautweichteilmantel und die Bandstrukturen der Nase - insbesondere im Bereich der Nasenspitze - erhält (s. o.). Allerdings betonen Saban und Daniel, dass die Patienten sehr genau ausgewählt werden müssen und dies sicherlich keine Technik für alle Höckernasen sei. 
11.1.1 Qualität der äußeren bzw. ästhetischen Ergebnisse Insgesamt beschreiben alle Operateure die zügigere postoperative Heilungsphase bei geringerer Schwellungstendenz, und dass die äußeren Ergebnisse sehr zufriedenstellend sind. Eine Kombination aus Preservation- und Piezotechnik ist mittlerweile auch schon in der Erprobungsphase und wurde als Fallbeschreibung durch Göksel und Saban 2019 publiziert [125].

\subsubsection{Qualität der funktionalen bzw. endonasalen Ergebnisse} Entsprechende Ergebnisse im Kurz- wie Langzeitbereich fehlen hier völlig. Sie stehen sicherlich auf der Agenda, um auch im Bereich Atmungsqualität die Leistungsfähigkeit der Preservation ggf. zu unterstreichen.

\subsubsection{Qualität der subjektiven Patienteneinschätzung}

Die bisher beschrieben Äusserungen der Patienten, die durch die Operateure genannt werden, waren positiv. Entsprechende PROMs sind leider noch nicht durchgeführt worden und können wohl auch erst in der Beurteilung einer Langzeitanalyse zu veritablen Ergebnissen führen.

\section{Patient Reported Outcomes (PROs)}

Maße für die klinische Wirksamkeit spiegeln typischerweise Ergebnisse wider, die für Patienten wichtig sind, wie etwa Symptome, Funktion, Optik oder Morbidität.

In den letzten Jahrzehnten hat sich erfreulicherweise die Sichtweise etabliert, dass Behandlungen nicht nur klinisch wirksam und rentabel, sondern auch für die Patienten akzeptabel und tatsächlich wünschenswert sein sollten. Messungen über die klinische Wirksamkeit können uns nicht sagen, wie ein Patient sich fühlt, denkt oder was er mit einer Behandlung erreichen möchte. Das Messen dieses Elements der Akzeptanz erfordert eine auf Patienten beruhende Evidenz, die das Wohlbefinden mit der Behandlung und dem Ergebnis einschließt.

Zu diesem Zweck wird ein zunehmender Fokus auf die Entwicklung von Patient Reported Outcomes (PROs) gelegt, die auf der Wahrnehmung einer Krankheit und ihrer Behandlung durch den Patienten beruhen. Patient Reported Outcome Measures (PROMs) sind Instrumente, die zur Messung und Erfassung von Daten zu PROs verwendet werden und haben insbesondere seit 2011 einen deutlichen Boom in den Publikationen erlebt [126].

Wir unterscheiden generell für alle PROMs 2 Kategorien. Einerseits generische PROM-Instrumente (z. B. SF-36, EQ-5D), die messen die allgemeine gesundheitsbezogene Lebensqualität und können unabhängig von der Erkrankung eingesetzt werden. Die Ergebnisse sind über verschiedene Patienten- oder Bevölkerungsgruppen hinweg vergleichbar. Andererseits die krankheitsspezifischen PROM-Instrumente (z. B. ROE, FROI-17, Oxford Knee Score), die messen den Schweregrad einer spezifischen Erkrankung oder einen bestimmten Aspekt einer Erkrankung oder eines Organs [127].

Die spezifische Befragung zeigt möglicherweise relevante Details auf, die von einem generischen Instrument nicht erfasst worden wäre. Ob ein generisches oder spezifisches Instrument gewählt werden soll, hängt von der Zielsetzung und Fragestellung ab.
Patient Reported Outcomes sind wichtig, da sie die Patientenperspektive der Behandlung und des Ergebnisses liefern, die durch die reine Klinik nicht erfasst wird, aber für den Patienten und seine Wahrnehmung genauso wichtig, wie ein wissenschaftliches Ergebnis ist.

Typischerweise werden hierbei systematisch folgende Dimensionen erhoben [128]:

- erreichter Gesundheitszustand

- Genesungsprozess (Geschwindigkeit, Qualität der Behandlungskette)

- Nachhaltigkeit

Im Gegensatz zu klinischen Standardergebnissen geben uns PROs einen einzigartigen Einblick darin, wie sich eine Therapie oder Operation insbesondere auch im äußeren Ergebnis nach einer Rhinoplastik auf den Patienten auswirken kann.

Personen mit genau den gleichen Gesundheitszuständen, Diagnosen oder Befindlichkeiten haben unterschiedliche Wahrnehmungen davon, da jeder einzelne andere Faktoren der Wahrnehmung über die Zufriedenheit eines Ergebnisses hat. PRO-Daten sind wichtig, da sie sich stärker auf den realen Nutzen konzentrieren, der für die Patienten von Bedeutung ist.

Die Fähigkeit, das Wohlbefinden als Ergebnis zu messen, wird in klinischen Situationen besonders wichtig, in denen das primäre Ziel der Behandlung das Wohlbefinden des Patienten (z. B. Rhinoplastik) und nicht die Verlängerung der Lebenserwartung ist. Die Verwendung von PROMs vor, während und nach einer Behandlung zeigt Veränderungen auf der individuellen Patientenebene auf: z. B. verbesserte körperliche Funktionsfähigkeit, höhere Lebensqualität [129].

PROs müssen sorgfältig definiert werden, damit sie die Informationen erfassen, die für die Patienten wichtig sind. Diese Auskünfte müssen auch exakt und soweit wie möglich auf eine Art und Weise gemessen werden, damit sie mit anderen Ergebnissen vergleichbar sind. Das bedeutet im Umkehrschluss, dass es sehr wichtig ist, wie eine Frage formuliert ist, wann die Fragen an den Patienten gestellt werden, wie die Antworten erfasst werden und wie die Daten interpretiert werden. Das heißt je präziser und spezifischer die Frage formuliert ist, umso besser erhält man detailliertere Informationen aus Patientensicht. Die Definition der jeweiligen PROs bestimmt das Messziel, wie z. B. ein Symptom, eine Wirkung, ein äußeres Merkmal usw. [130,131].

Die wichtigsten anhand von PROs gemessenen Konzepte sind:

- Gesundheitsbezogene Lebensqualität (HRQoL)

Die HRQoL ist mehrdimensional und repräsentiert die Patientenbewertung einer Erkrankung und ihrer Behandlung in seinem Alltag einschließlich der körperlichen, psychischen, sozialen Funktion und der Emotion, dem Wohlbefinden, der Vitalität, des Gesundheitszustands usw.

\section{- Patientenzufriedenheit}

Die Bewertung der ambulanten oder stationären Einrichtung sowie der einzelnen Behandlungen, der Leistungserbringer inkl. aller Angehörigen der Gesundheitsberufe usw.

- Körperliche Funktionen

Körperliche Ein- und Beschränkungen in Ruhe und bei Aktivität einschließlich der Selbstversorgung und anderer täglicher Aktivitäten, wie Laufen, Mobilität, Schlaf, Sex usw.

- Psychischer Zustand 
Positive oder negative Emotionen, kognitives Denken einschließlich Zorn, Aufmerksamkeit, Selbstwertgefühl, Gefühl von Wohlbefinden, Kummer usw.

\section{- Zeichen und Symptome}

Berichte körperlicher und psychischer Symptome oder Gefühle, die nicht direkt zu beobachten sind einschließlich geistiger und körperlicher Kraft und Ermüdung, Übelkeit, Reizbarkeit usw.

- Soziale Kompetenz

Einschränkungen bei der Arbeit, in der Schule, im Freundes- und Familienkreis usw., sowie die aktive Teilnahme am gesellschaftlichen Leben

- Therapietreue (postoperative Nachsorge)

Berichte oder Beobachtungen zur aktiven postoperativen Compliance

- Nützlichkeit

Die Nützlichkeit bzw. die Brauchbarkeit ist die nutzbringende Beschaffenheit von etwas zur Befriedigung von Bedürfnissen. In der Gesundheitsökonomie wird die Patientenpräferenz anhand der Nützlichkeit gemessen, z. B. wie wichtig verschiedene Faktoren für Patienten sind, wie bspw. Symptome, Schmerzen und psychische Gesundheit. Damit kann die Auswirkung neuer Behandlungen auf diese Faktoren und somit auf die Lebensqualität ermittelt werden. Sie ist sowohl ein gängiger Ansatz zur Bewertung von neuen Therapieansätzen als auch zur Beurteilung, ob Behandlungen von Kostenträgern übernommen werden sollen.

Ein qualitativ hochstehendes PROM-Instrument ist valide, liefert konsistente, reproduzierbare Angaben über die Wirkung der Behandlung und reagiert auf Veränderungen. Bei der Wahl des PROM-Instruments ist es wichtig, dass die Eigenschaften der Personengruppe, worauf die Evidenz basiert (Alter, Geschlecht, Krankheit usw.), vergleichbar sind mit der zu untersuchenden Gruppe. Zur Entwicklung, Beurteilung, Implementierung und Berichterstattung von PROMs sind multiple Handbücher und Publikationen verfügbar. Das heisst aber auch, dass die Veränderung der Lebensqualität in PROMs häufig nicht optimal dargestellt wird, denn häufig haben die Studien keine Kohorte, sind nicht prospektiv und verfügen nur über einen kurzen Nachbeobachtungszeitraum [126, 132-134].

\subsubsection{Qualität der äußeren bzw. ästhetischen Ergebnisse und der Lebensqualität}

Die Qualitätsanalysebögen sind im Bereich der Rhinoplastik meistens eine Kombination aus innerem und äußerem Ergebnis mit dem Schwerpunkt der postoperativen Funktion. Die einzige Ausnahme im krankheitsspezifischen Bereich ist die „Rhinoplasty Outcome Evaluation (ROE)“ mit einem äußerlichen Frageschwerpunkt. Die ROE wurde 2001 durch Alsarraf et al. publiziert [135].

Die ROE ist im Moment das einzige auf das äußere Ergebnis der Rhinoplastik fokussierte krankheitsspezifische PROM. Allerdings muss man anmerken, dass der Fragebogen auch Nachteile hat, da er nur über 6 Fragen insgesamt, von denen 5 auf das Äußere zielen, verfügt. Das heißt in Sensitivität und Spezifität hat die ROE definitive Schwächen und erfüllt nicht optimal die o.g. Bedingungen an einen krankheitsspezifischen PROM.

In 2 retrospektiven Arbeiten konnte für die ROE nach 5 Monaten [136] und 36 Monaten [137] eine Verbesserung um 36,7 Punkte im Mittel nachgewiesen werden.
In einer der größten Follow-up-Arbeiten (60 Monate) belegte die Gruppe um Bulut und Baumann, dass die ROE nach einem und 5 Jahren eine deutliche postoperative Verbesserung der äußeren Erscheinung und Lebensqualität nachweisen konnte [138]. Sie ist damit die einzige Studie, die überhaupt diesen langen Nachbeobachtungszeitraum für den Bereich der Septorhinoplastik aufgearbeitet hat. Die Daten legen dar, dass retrospektive Studien ähnliche Ergebnisse, wie die prospektiven Analysen zeigen, allerdings ist die Validität bei prospektiven Studien natürlich deutlich höher als bei retrospektiven Studien (Stichwort: response shift bias, s.u.).

Eine Interimsstellung unter den PROMs nimmt der FACE-Q ein, da er sich nicht auf die Nase, sondern auf Veränderungen des Gesichts konzentriert und somit auch für den Bereich Lidplastik, Facelifting usw. zur Anwendung kommt [139].

Dies macht umsomehr Sinn, da die Nase mitten im Gesicht ist und alles andere in der Wahrnehmung der Patienten überdeckt. In der Weiterentwicklung des FACE-Q gibt es ein Nasenmodul, was 19 Fragen umfasst, wobei zehn Fragen die Nase, 4 Fragen nachteilige Effekte der Operation und fünf Fragen die Nasenlöcher umfassen. Daraus konnte ebenfalls evaluiert werden, dass die äußere Veränderung im Gesicht auf alle Bereiche einen signifikant positiven Effekt hat ( $\mathrm{Er}$ scheinung, Lebensqualität, seelisches Wohlbefinden usw.) [140].

Der generische PROM „Short-Form 36 Health Survey (SF-36)“, der das allgemeine körperliche Befinden analysiert [141], zeigte die Verbesserung ein Jahr postoperativ in den Bereichen rollenspezifische Wirkung und kognitive Gesundheit. Fünf Jahre postoperativ konnten signifikante Verbesserungen gegenüber dem präoperativen Befund in den Bereichen Körperfunktion, rollenspezifische Wirkung, kognitive Gesundheit, körperliche Schmerzen, Vitalität und soziale Funktion nachgewiesen werden. Allerdings gab es bei SF-36 keinerlei signifikante Veränderung zwischen dem 12. und 60. Monat postoperativ. Dies ist äußerst interessant, da eine Verbesserung zum präoperativen Zustand jeweils nachweisbar war, was den Schluss zuliese, dass der postoperativen Erfassung mit SF-36 Grenzen gesetzt sind. Eine Korrelation zwischen SF-36 und ROE konnte für alle Untersuchungszeiträume nicht nachgewiesen werden (Bild 3). Dies könnte an den sehr unspezifischen Fragen und der geringen Fragenanzahl der ROE liegen, die damit ihren Schwachpunkt einer krankheitsspezifischen PROM offenbahrt (s. o.).

\subsubsection{Qualität der funktionellen bzw. endonasalen Ergeb- nisse und der Lebensqualität}

Im funktionellen Bereich nach Rhinoplastik spielen insbesondere 2 PROMs eine wesentliche Rolle. NOSE (Nasal Obstruction Symptom Evaluation), der 2004 durch Stewart et al. etabliert wurde und primär den funktionellen und obstruktiven Aspekt beleuchtet und eine Verbesserung der Lebensqualität nach operativer Sanierung einer behinderten Nasenluftpassage belegen konnte [142].

Eine neuerer PROM wurde 2018 vorgestellt, der eine starke Korrelation zu NOSE hat aber nicht im ästhetischen Bereich diese Gewichtung aufweisen kann. SCHNOS soll der Vollständigkeit halber erwähnt werden, muss aber aufgrund der Kürze seiner Verfügbarkeit noch weitere Daten liefern. Er beinhaltet vier Fragen zur Atmung, 5 Fragen zur Nasenform und eine Frage zum Selbstbewußsein der Patienten [143].

Darüberhinaus FROI-17 der 2014 durch die Gruppe um Baumann et al. initiiert und etabliert wurde [144]. FROI-17 ist breiter aufgestellt, 
da er optische, wie auch funktionale Daten berücksichtigt. In der Analyse der Funktionsergebnisse waren für die Nasenfunktion eine signifikante Veränderung des Patientenempfindens 12 und 60 Monate postoperativ nachzuweisen ist [138]. Des Weiteren konnten Sie belegen, dass Patienten mit einer präoperativen Schiefnase postoperativ signifikant zufriedener, als Patienten mit einer geraden präoperativen Nase waren. Des Weiteren war eine Korrelation zwischen dem generischen PROM SF-36 und dem krankheitsspezifischen PROM FROI-17, im Gegensatz zum ROE, nachzuweisen ( $>$ Abb. 3) [85].

Diese Daten der FROI-17 Studie der inneren und äußeren Nase sind umso gewichtiger, da es sich hierbei um eine prospektive Studie und nicht um eine, wie meist, retrospektive Studie handelt, die immer der Gefahr eines Verzerrungseffekt durch die Beurteilung der präoperativen Situation im Nachhinein unterliegt (response shift bias).

Wir können daraus erkennen, dass es auch langfristig zu einer signifikanten Verbesserung der Lebensqualität aus Sicht der Patienten kommt, die sowohl das innere als auch das äußere der Nase berücksichtigt.

\subsubsection{Qualität der subjektiven Patienteneinschätzung bei PROM in der Rhinoplastik}

Zunächst muss, wie für alle ästhetischen Patienten, die eigene Körperwahrnehmung berücksichtigt werden. Eine Verbesserung der seelischen Gesundheit durch ästhetische Eingriffe konnte schon Mitte der 1990er Jahre nachgewiesen werden [145]. Das häufigste mentale Krankheitsbild, das einem in der plastischen Chirurgie begegnet, ist die Dysmorphophobie. Aktuelle Metaanalysen konnten nachweisen, dass im Mittel 15\% aller Patienten, mit dem Wunsch der äußeren Veränderung, eine Dysmorphophobie haben. Das wäre mehr als jeder sechste Patient. In der Rhinoplastik liegt diese Zahl noch höher und wird zwischen 21 und $48 \%$ angegeben.

Dies wirkt sich natürlich auch in den Ergebnissen und in der Interpretation von PROMs aus [146]. Picavet et al. konnten einen eindeutigen Zusammenhang zwischen einer fehlerhaften Körperwahrnehmung und dem Wunsch zur Rhinoplastik belegen [147]. Felix et al. konnten darüberhinaus aufzeigen, dass Patienten mit einer körperdysmorphen Störung von einer Rhinoplastik profitieren, sie aber prä- und postoperativ mit deutlich schlechteren Werten abschneiden, als die Kontrollgruppe ohne diese mentale Störung [148]. Darüberhinaus muss in der Einschätzung aller Fragetools berücksichtigt werden, dass Nasenpatienten meistens weiblich, jung und gesund sind und ansonsten üblicherweise keine weiteren Einschränkungen haben. Somit wirkt sich die Verbesserung eines körperlichen Befundes direkter auf alle Bereiche des Lebens aus. Schwitzer et al. legten in einer demographischen Analyse dar, dass die Zufriedenheit über eine Verbesserung des äußerlichen Erscheinungsbildes und der Lebensqualität von Alter, Geschlecht, Rasse und Einkommen abhängig ist. Weiße, weibliche Patienten unter 35 Jahre waren deutlich zufriedener mit ihrer postoperativen Lebensqualität und ihrem Äußeren als alle anderen Gruppen [44].

Des Weiteren ist die Zeitachse im Follow-up ein wesentlicher Punkt in den Analysen der PROMs. Die positive Veränderung auf Körper, Geist und die Lebensqualität ist insbesondere in den ersten 12 Monaten nachzuweisen [149]. Eine Metaanalyse von Yang et al. konnte 2018 in der Sichtung aller rhinospezifischen PROMs dies verifizieren und die Verbesserung der Lebensqualität insbesondere in den ersten 12 Monaten und bei jungen Patienten nachweisen [150].

\begin{tabular}{|c|c|c|c|c|c|c|c|c|c|}
\hline & \multicolumn{2}{|c|}{ Preoperatively } & \multicolumn{2}{|c|}{$\begin{array}{l}\text { 1-year postop- } \\
\text { eratively }\end{array}$} & \multicolumn{2}{|c|}{$\begin{array}{l}\text { 5-year postop- } \\
\text { eratively }\end{array}$} & \multirow[t]{2}{*}{ p (preop vs. 1-year) } & \multirow[t]{2}{*}{ p (preop vs. 5-year) } & \multirow[t]{2}{*}{$\begin{array}{c}p \text { (1-year } \\
\text { vs. } 5 \text {-year) }\end{array}$} \\
\hline & Mean & SD & Mean & SD & Mean & SD & & & \\
\hline \multicolumn{10}{|l|}{ FROI-17 } \\
\hline Overall score & 32.2 & 17.5 & 20.2 & 18.3 & 13.7 & 17.3 & $<0.0001$ & $<0.0001$ & 0.048 \\
\hline Nasal symptoms & 31.7 & 17.0 & 21.5 & 19.7 & 14.7 & 18.8 & 0.001 & $<0.0001$ & 0.05 \\
\hline General symptoms & 32.9 & 22.8 & 20.0 & 20.7 & 12.9 & 17.9 & 0.0002 & $<0.0001$ & 0.043 \\
\hline Self-confidence & 30.8 & 26.5 & 16.9 & 21.8 & 14.3 & 23.7 & 0.0002 & $<0.0001$ & 0.55 \\
\hline \multicolumn{10}{|l|}{ ROE } \\
\hline Overall score & 39.8 & 15.2 & 68.5 & 17.8 & 75.1 & 24.0 & $<0.0001$ & $<0.0001$ & 0.15 \\
\hline \multicolumn{10}{|l|}{ SF-36 } \\
\hline Physical functioning & 84.0 & 19.4 & 91.4 & 13.3 & 94.4 & 13.3 & 0.05 & 0.001 & 0.23 \\
\hline Role-functioning physical & 75.5 & 35.7 & 89.1 & 23.7 & 90.1 & 25.6 & 0.02 & 0.004 & 0.85 \\
\hline Bodily pain & 80.3 & 26.5 & 83.6 & 23.2 & 89.5 & 22.7 & 0.49 & 0.026 & 0.26 \\
\hline General health & 66.0 & 21.0 & 70.9 & 20.0 & 66.4 & 19.5 & 0.24 & 0.90 & 0.31 \\
\hline Vitality & 53.1 & 19.6 & 57.2 & 19.6 & 62.8 & 19.7 & 0.30 & 0.004 & 0.20 \\
\hline Social functioning & 76.0 & 25.2 & 80.5 & 24.6 & 87.5 & 20.7 & 0.38 & 0.003 & 0.18 \\
\hline Role-functioning emotional & 80.2 & 33.4 & 85.4 & 31.6 & 88.7 & 26.9 & 0.43 & 0.09 & 0.63 \\
\hline Mental health & 61.8 & 19.2 & 70.6 & 18.4 & 71.0 & 18.2 & 0.024 & 0.004 & 0.90 \\
\hline
\end{tabular}

$S D$ standard deviation, $p p$ value, vs versus

- Abb. 3 Präoperative Bewertung durch ROE, FROI-17 und SF-36 zu einem und fünf Jahre postoperativ, sowie ein Jahr zu fünf Jahre postoperativ . Aus: Bulut OC, Wallner F, Oladokun D et al. Long-term quality of life changes after primary septorhinoplasty. Quality of life research : an international journal of quality of life aspects of treatment, care and rehabilitation 2018; 27: $987-991$ 
Sarver und Kollegen hatten diesen Bereich enger gefasst, in dem sie in einer prospektiven Studie belegten, dass der positive Wandel bei ästhetischen Operationen im Allgemeinen (Nase, Lider, Brust usw.) sich unabhängig vom Patientengut primär in den ersten 3 Monaten abspielt [151]. Eine Verbesserung auf die Lebensqualität war nach 60 Monaten bei keiner einzigen Studie bisher nachweisbar [152].

Auf der einen Seite wirken sich die funktionalen und optischen Verbesserungen positiv in den Beurteilungsbögen (SF-36, FROI-17 und ROE), insbesondere im kürzeren Follow-up von einem Jahr aus. Allerdings zeigen sich die funktionalen Verbesserungen mit der Auswirkung auf Resozialisierung, Selbstvertrauen und Selbstwertgefühl erst deutlich später, was sich in der Erfassung der Langzeitdaten des FROI-17 widerspiegelt; im Gegensatz zum ROE, der keinerlei positive Langzeitveränderungen nachweisen kann. Ein wesentlicher Aspekt, den Bulut et al. beweisen konnten entspricht den schon oben genannten Daten, dass nämlich die Art der Prozedur in keinster Weise die Lebensqualität oder das Körperempfinden positiv oder negativ beeinflussen. Dies ist umso interessanter, da bei deren Patientengut 2/3 Drittel geschlossen und 1/3 offen operiert wurde, was dem allgemeinen Trend im Moment entgegentritt [153].

\section{FAZIT}

1. Die Qualität in der plastisch-ästhetischen Rhinoplastik ist von vielen unterschiedlichen Faktoren abhängig

2. Wie in allen Bereichen muss sich die Rhinoplastik den Einflüssen von Institutionen, Kostenträgern, Patienten und den Medien (primär Internet und Social media) stellen

3. Nachweisbare und fassbare Qualität wird von den einzelnen Gruppen jeweils anders definiert und erwartet

4. Wirtschaftlichkeit und Qualität schließen sich nicht aus, aber bedingen sich definitiv nicht gegeneinander

5. Die Qualität der Rhinoplastik ist bei Veränderungen des äußeren Erscheinungsbildes häufig von weichen Faktoren und dem ästhetischen Geschmack des Patienten abhängig

6. Die Qualität der Rhinoplastik ist nach heutigem Wissen nicht von der Art des Zugangs, der Osteotomietechnik oder anderer einzelner Schritte abhängig, sondern dem äußerlichen und funktionellen Endergebnis geschuldet, das sich in den generischen und krankheitsspezifischen PROMs widerspiegelt.

7. Ergebnisqualität ist höher als Prozedurqualität zu bewerten.

8. Die Qualitätssicherung und der Qualitätsnachweis können gegenüber allen Bereichen und Institutionen nur über eine wissenschaftliche Aufarbeitung in Studien gelingen, die in der Levelsteigerung der Leitlinien und in evidenzbasierten Daten münden sollte.

9. Das Ziel sollte die seriöse Etablierung von prospektiven Vergleichsstudien sein, wie sie in der Medikamenten- oder Krebstherapie heute selbstverständlich sind, um sich gegenüber den Kostenträgern besser aufstellen zu können und um unabhängiger von Industrieeinflüssen zu sein.
Interessenkonflikt

Der Autor gibt an, dass kein Interessenkonflikt besteht.

\section{Literatur}

[1] Parsa-Parsi RW. The Revised Declaration of Geneva: A Modern-Day Physician's Pledge. JAMA 2017; 318: 1971-1972. doi:10.1001/ jama.2017.16230

[2] Medizin ÄZfQid. Gemeinsame Stellungnahme von BÄK und KBV zur Qualitätsverbesserung im Gesundheitswesen. In: Deutschland: 1998

[3] Institute of Medicine (U.S.) Division of Health Care Services., Lohr KN, Institute of Medicine (U.S.). Committee to Design a Strategy for Quality Review and Assurance in Medicare. et al. Medicare : a strategy for quality assurance. Washington, D.C: National Academy Press; 1990

[4] Cassier-Woidasky AK. What do people expect from their hospital? Results of a visitor survey at open house in a general hospital. Pflege 1998; 11: 248-254

[5] Höhmann UM-M, Schulz G, Qualität B. durch Kooperation - Gesundheitsdienste in der Vernetzung In: Pflege. Frankfurt: Mabuse; 1997: 248-254

[6] Schmidt C, Moller J, Reibe F et al. Patient satisfaction with inpatient care. Evaluation, methods and special features. Dtsch Med Wochenschr 2003; 128: 619-624. doi:10.1055/s-2003-38055

[7] Nolting HdW, J. Der Patient vor der Wahl. Durch mehr Wissen zu mehr Verantwortung. Ergebnisse der Janssen-Cilag Bevölkerungsbefragung 2002. Neuss: Eigenverlag Janssen-Cilag; 2002

[8] Stock S, Hertle D, Veit C. Patient-centredness and Quality of Care in Germany in International Comparison - Results of a Telephone Survey of Patients in 11 Countries. Gesundheitswesen 2015; 77: 761-767. doi:10.1055/s-0034-1387757

[9] Gaydoul T. Qualitätsberichte von Krankenhäusern: eine empirische Analyse aus informationsökonomischer Sicht. Wiesbaden: Gabler Zugl.: Hohenheim; 2009: 256 S.

[10] Klemperer D. Wie Ärzte und Patienten Entscheidungen treffen. Berlin: Wissenschaftszentrum Berlin für Sozialforschung (WZB); 2003

[11] Dierks M-L. Bürger- und Patientenorientierung im Gesundheitswesen. Berlin: Robert Koch-Institut; 2006

[12] Kidd J, Patel V, Peile E et al. Clinical and communication skills. BM] 2005; 330: 374-375. doi:10.1136/bmj.330.7488.374

[13] Little P, Dorward M, Warner G et al. Importance of patient pressure and perceived pressure and perceived medical need for investigations, referral, and prescribing in primary care: nested observational study. BMJ 2004; 328: 444. doi:10.1136/bmj.38013.644086.7C

[14] Kahrs MS.T Medizinische Versorgung und Patientenbedürfnisse. Berlin: Wissenschaftlicher Verlag; 2003

[15] Harter M. Shared decision making - from the point of view of patients, physicians and health politics is set in place. Z Arztl Fortbild Qualitatssich 2004; 98: 89-92

[16] Dierks M-L. Patientensouveränität: der autonome Patient im Mittelpunkt. Stuttgart: Akad. für Technikfolgenabschätzung in Baden-Württemberg; 2001

[17] [Anonym] Guide to Quality Assurance.In Chicago, USA: Joint Commission on Accreditation of Healthcare Organizations (JCAHO). 1998

[18] Van der Stuyft P, Unger JP. Improving the performance of health systems: the World Health Report as go-between for scientific evidence and ideological discourse. Trop Med Int Health 2000; 5: 675-677

[19] al. GMJWTCALe Qualitätsindikatoren in Deutschland. Positionspapier des Expertenkreises Qualitätsindikatoren beim Ärztlichen Zentrum für Qualität in der Medizin (ÄZQ), Berlin. Z Arztl Fortbild Qualitatssich 2005; 99: 329-331 
[20] Geraedts M, Selbmann HK, Ollenschlager G. Assessment of methodological quality of clinical performance measures. Z Arztl Fortbild Qualitatssich 2002; 96: 91-96

[21] Wölker T. Qualitätsmanagement in der Arztpraxis - So managen Sie Qualität. Neu-Isenburg: Ärzte-Zeitung-Verl; -Ges. 2002

[22] Medizin ÄZfQid Woran erkennt man eine gute Arztpraxis? - Checkliste für Patientinnen und Patienten. 4. Aufl. Aufl. Berlin: ÄZQ; 2015

[23] Blanke BK.H Die Ökonomisierung der Gesundheitspolitik: Von der Globalsteuerung zum Wettbewerbskonzept im Gesundheitswesen. Leviathan 1996; 24: 512-538

[24] Shulman LN, Palis BE, McCabe R et al. Survival As a Quality Metric of Cancer Care: Use of the National Cancer Data Base to Assess Hospital Performance. J Oncol Pract 2018; 14: e59-e72. doi:10.1200/ JOP.2016.020446

[25] Helou A, Schwartz FW, Ollenschlager G. Qualitätsmanagement und Qualitätssicherung in Deutschland. Bundesgesundheitsblatt Gesundheitsforschung Gesundheitsschutz 2002; 45: 205-214. doi:10.1007/s00103-001-0372-1

[26] Herholz H. Qualitätssicherung und Qualitätsmanagement in der ambulanten Versorgung am Beispiel Hessen. Bundesgesundheitsblatt Gesundheitsforschung Gesundheitsschutz 2002; 45: 249-259. doi:10.1007/s00103-002-0379-2

[27] Schillinger D, Piette J, Grumbach K et al. Closing the loop: physician communication with diabetic patients who have low health literacy. Arch Intern Med 2003; 163: 83-90. doi:10.1001/archinte.163.1.83

[28] Ortmann K. Discrepancies between physicians and patients with long-term functional somatoform disorders as a central treatment problem. How the quality of assistance can be improved. Z Arztl Fortbild Qualitatssich 2000; 94: 708-712

[29] Hoffrage U, Kurzenhauser S, Gigerenzer G. How can one improve the understanding and communication of the importance of medical test results?. Z Arztl Fortbild Qualitatssich 2000; 94: 713-719

[30] [Anonym] Hausärztliche Gesprächsführung. Hausärztliche Leitlinie. In: Leitliniengruppe Hessen. Köln: PMV Forschungsgruppe; 2006

[31] Schulz von Thun F. Miteinander reden. 14. Aufl. Reinbek: Rowohlt; 1998

[32] Ollenschlager G, Marshall C, Qureshi S et al. Improving the quality of health care: using international collaboration to inform guideline programmes by founding the Guidelines International Network (G-I-N). Qual Saf Health Care 2004; 13: 455-460. doi:10.1136/ qhc. 13.6.455

[33] Kirchner H, Fiene M, Ollenschlager G. Dissemination and implementation of guidelines in public health: current state in July 2001. Dtsch Med Wochenschr 2001; 126: 1215-1220. doi:10.1055/s-2001-18003

[34] Lelgemann M, Ollenschlager G. Evidence based guidelines and clinical pathways: complementation or contradiction?. Internist (Berl) 2006; 47: 690 692-697. doi:10.1007/s00108-006-1652-5

[35] Grimshaw JM, Thomas RE, MacLennan G et al. Effectiveness and efficiency of guideline dissemination and implementation strategies. Health Technol Assess 2004; 8: iii-iv 1-72

[36] Encke AKI, Selbmann HK, Hoppe D et al. Das Deutsche Instrument zur methodischen Leitlinien-Bewertung (DELBI). Dtsch Arztebl 2005; 102: 1912-1913. doi:1

[37] Kirchner HO.G Implementierung von Leitlinien. Netze auf dem Weg zur evidenzbasierten Medizin. In Tophoven CL L, Hrsg. Integrierte Versorgung. Köln: Dt. Ärzte-Verl; 2002: 63-106

[38] Bundesvereinigung BK. Beurteilungskriterien für Leitlinien in der medizinischen Versorgung - Beschlüsse der Vorstände der Bundesärztekammer und Kassenärztlicher Bundesvereinigung. Dtsch Ärztebl 1997; 94: A2154-A2155

[39] Schubert IL, Kirchner M, von Ferber HC et al. Handbuch zur Entwicklung regionaler Leitlinien. Norderstedt: BoD, Books on Demand 2006
[40] Kirchner HS, Weingart S, Ollenschläger O.G Methoden und Techniken der Evidenzbasierten Medizin. In: Handbuch Qualitätszirkel. Köln: Kassenärztliche Bundesvereinigung; 2003

[41] Fessler J, Gross J, Papendick H et al. Qualitative and economc impact of implementing GP guidelines in a medical practitioners' network. Z Arztl Fortbild Qualitatssich 2006; 100: 107-112

[42] Pizzi LTG.N Promoting a Culture of Safety. Making Health Care Safer: A Critical Analysis of Patient Safety Practices. Evidence. In: (AHRQ) AfHRaQ., Hrsg.Evidence Report/Technology Assessment; Rockville: Ed Nash DB2001

[43] Rosenthal J, Riley T. National Academy for State Health Policy (U.S.) et al Patient safety and medical errors: a road map for state action. Portland, ME: National Academy for State Health Policy; 2001

[44] Schwitzer JA, Albino FP, Mathis RK et al. Assessing Demographic Differences in Patient-Perceived Improvement in Facial Appearance and Quality of Life Following Rhinoplasty. Aesthetic surgery journal 2015; 35: 784-793. doi:10.1093/asj/sjv066

[45] Bitzer EMD.ML Wie kann man Erwartungen und Zufriedenheit der Patienten im Qualitätsmanagement berücksichtigen? - Erhebungsverfahren und Erfahrungen aus der ambulanten Versorgung.In Gesundheit DBf, Hrsg. Qualitätsmanagement in der Arztpraxis. Baden-Baden: Nomos; 1999: 125-184

[46] Oladokun D, Baumgart A, Baumann I et al. Quality of Life Gain After Septorhinoplasty: An Analysis of Health Utility and Cost Utility Values Associated with Septorhinoplasty. Aesthetic plastic surgery 2018; 42: 1618-1624. doi:10.1007/s00266-018-1226-7

[47] Luk L], Steele TO, Mace JC et al. Health utility outcomes in patients undergoing medical management for chronic rhinosinusitis: a prospective multiinstitutional study. Int Forum Allergy Rhinol 2015; 5: 1018-1027. doi:10.1002/alr.21588

[48] Barton GR, Bankart ], Davis AC et al. Comparing utility scores before and after hearing-aid provision: results according to the EQ-5D, HUI3 and SF-6D. Appl Health Econ Health Policy 2004; 3: 103-105. doi:10.2165/00148365-200403020-00006

[49] Arnoldner C, Lin VY, Honeder C et al. Ten-year health-related quality of life in cochlear implant recipients: prospective SF-36 data with SF-6D conversion. The Laryngoscope 2014; 124: 278-282. doi:10.1002/lary.24387

[50] Feeny D, Wu L, Eng K. Comparing short form 6D, standard gamble, and Health Utilities Index Mark 2 and Mark 3 utility scores: results from total hip arthroplasty patients. Quality of life research: an international journal of quality of life aspects of treatment, care and rehabilitation 2004; 13: 1659-1670. doi:10.1007/s11136-004-6189-2

[51] Biggs TC, Fraser LR, Ward M] et al. Patient reported outcome measures in septorhinoplasty surgery. Annals of the Royal College of Surgeons of England 2015; 97: 63-65. doi:10.1308/00358841 $4 \times 14055925059075$

[52] Black W. An arithmetical and medical analysis of the diseases and mortality of the human species By. William Black MD. In: The second edition corrected and improved. ed. London: printed for the author by John Crowder: and sold by C. Dilly; 178912 ,ix, 11 ,265, 261 p., 262 leaves of plates

[53] Trohler U. „To improve the evidence of medicine“: arithmetic observation in clinical medicine in the eighteenth and early nineteenth centuries. Hist Philos Life Sci 1988; 10: Suppl 31-40

[54] Kunz R, Cox M. Lehrbuch evidenzbasierte Medizin in Klinik und Praxis. mit 85 Tabellen. 2., überarb. und erw. Aufl. Aufl. Köln: Dt. Ärzte-Verl; 2007

[55] Beckmann JS, Lew D. Reconciling evidence-based medicine and precision medicine in the era of big data: challenges and opportunities. Genome Med 2016; 8: 134. doi:10.1186/s13073-016-0388-7 
[56] Sackett DL, Rosenberg WM, Gray JA et al. Evidence based medicine: what it is and what it isn't. BMJ 1996; 312: 71-72. doi:10.1136/ bmj.312.7023.71

[57] Karaiskakis P, Bromba M, Dietz A et al. Reconstruction of nasal tip support in primary, open approach septorhinoplasty: A retrospective analysis between the tongue-in-groove technique and the columellar strut. European archives of oto-rhino-laryngology: official journal of the European Federation of Oto-Rhino-Laryngological Societies (EUFOS): affiliated with the German Society for Oto-Rhino-Laryngology - Head and Neck Surgery 2016; 273: 2555-2560. doi:10.1007| s00405-016-3911-y

[58] Ellis J, Mulligan I, Rowe J et al. Inpatient general medicine is evidence based. A-Team, Nuffield Department of Clinical Medicine. Lancet 1995; 346: 407-410

[59] Powell JA, Geddes JR. Evidence-based psychiatry. Br J Psychiatry 1997; 171: 586-587. doi:10.1192/bjp.171.6.586c

[60] Gill P, Dowell AC, Neal RD et al. Evidence based general practice: a retrospective study of interventions in one training practice. BM] 1996; 312: 819-821. doi:10.1136/bmj.312.7034.819

[61] Dietz A, Wiegand S, Kuhnt T et al. Laryngeal Preservation Approaches: Considerations for New Selection Criteria Based on the DeLOS-II Trial. Front Oncol 2019; 9: 625. doi:10.3389/fonc.2019.00625

[62] Sheen JH, Sheen AP. Aesthetic rhinoplasty. St. Louis: C. V. Mosby Co; 1978

[63] Joseph J. Nasenplastik und sonstige Gesichtsplastik nebst einem Anhang über Mammaplastik und einige weitere Operationen aus dem Gebiete der äusseren Körperplastik: ein Atlas und Lehrbuch. Leipzig: Kabitzsch; 1931

[64] Cakir B, Oreroglu AR, Dogan T et al. A complete subperichondrial dissection technique for rhinoplasty with management of the nasal ligaments. Aesthetic surgery journal 2012; 32: 564-574. doi:10.1177 /1090820X12445471

[65] Ozmen S, Eryilmaz T, Sencan A et al. Sliding alar cartilage (SAC) flap: a new technique for nasal tip surgery. Annals of plastic surgery 2009; 63: 480-485. doi:10.1097/SAP.0b013e31819538a8

[66] Gruber RP, Zhang AY, Mohebali K. Preventing alar retraction by preservation of the lateral crus. Plastic and reconstructive surgery 2010; 126: 581-588. doi:10.1097/PRS.0b013e3181de22d1

[67] Davis RE. Lateral crural tensioning for refinement of the wide and underprojected nasal tip: rethinking the lateral crural steal. Facial plastic surgery clinics of North America 2015; 23: 23-53. doi:10.1016/j.fsc.2014.09.003

[68] Palhazi P, Daniel RK, Kosins AM. The osseocartilaginous vault of the nose: anatomy and surgical observations. Aesthetic surgery journal 2015; 35: 242-251. doi:10.1093/asj/sju079

[69] Saban YP, Atlas d'Anatomie R. Chirurgicale de la Face et du Cou. Florence, Italy: SEE Editrice; 2009

[70] Goodman WS. External approach to rhinoplasty. Can J Otolaryngol 1973; 2: 207-210

[71] Anderson JR, Ries WR. Rhinoplasty: emphasizing the external approach. New York: Thieme; 1986

[72] Daniel RK. Rhinoplasty: creating an aesthetic tip. A preliminary report. Plastic and reconstructive surgery 1987; 80: 775-783

[73] Gunter JP, Rohrich RJ. External approach for secondary rhinoplasty. Plastic and reconstructive surgery 1987; 80: 161-174. doi:10.1097/00006534-198708000-00001

[74] Aiach G. Mini-forum: rhinoplasty by external approach. External or endonasal approach for rhinoplasty?. Annales de chirurgie plastique et esthetique 1992; 37: 498-509

[75] Fritz K. „Open approach“-,progress“ back the beginnings of septorhinoplasty. Hno 2000; 48: 562-567
[76] Durante B], Porubsky ES. Reducing columella scarring in open septorhinoplasty. The Laryngoscope 1986; 96: 810-811

[77] Friedman GD, Gruber RP. A fresh look at the open rhinoplasty technique. Plastic and reconstructive surgery 1988; 82: 973-982. doi:10.1097/00006534-198812000-00006

[78] Yagmur C, Ak S, Engin MS et al. Columellar Scar Perception in Open Rhinoplasty. Interplay of Scar Awareness, Body Cathexis and Patient Satisfaction. Aesthetic plastic surgery 2017; 41: 153-160. doi:10.1007/s00266-016-0719-5

[79] Constantian MB. Differing characteristics in 100 consecutive secondary rhinoplasty patients following closed versus open surgical approaches. Plastic and reconstructive surgery 2002; 109: 2097-2111. doi:10.1097/00006534-200205000-00048

[80] Daniel RK, Palhazi P. The Nasal Ligaments and Tip Support in Rhinoplasty: An Anatomical Study. Aesthetic surgery journal 2018; 38: 357-368. doi:10.1093/asj/sjx192

[81] Pitanguy I. Surgical Importance of a Dermocartilaginous Ligament in Bulbous Noses. Plastic and reconstructive surgery 1965; 36: 247-253. doi:10.1097/00006534-196508000-00014

[82] Toriumi DM, Mueller RA, Grosch T et al. Vascular anatomy of the nose and the external rhinoplasty approach. Arch Otolaryngol Head Neck Surg 1996; 122: 24-34

[83] Berghaus A. Modern Rhinoplasty: Is There a Place for the Closed Approach? Facial plastic surgery: FPS 2016; 32: 402-408. doi:10.1055/s-0036-1585422

[84] Gokce Kutuk S, Arikan OK. Evaluation of the effects of open and closed rhinoplasty on the psychosocial stress level and quality of life of rhinoplasty patients. Journal of plastic, reconstructive \& aesthetic surgery : JPRAS 2019; 72: 1347-1354. doi:10.1016/j. bjps.2019.03.020

[85] Bulut OC, Wallner F, Hohenberger R et al. Quality of life after primary septorhinoplasty in deviated-and non-deviated nose measured with ROE, FROI-17 and SF-36. Rhinology 2017; 55: 75-80. doi:10.4193| Rhin 16.243

[86] Saban Y, Daniel RK, Polselli R et al. Dorsal Preservation: The Push Down Technique Reassessed. Aesthetic surgery journal 2018; 38: 117-131. doi:10.1093/asj/sjx180

[87] Daniel RK. The Preservation Rhinoplasty: A New Rhinoplasty Revolution. Aesthetic surgery journal 2018; 38: 228-229. doi:10.1093/asj/sjx258

[88] Toriumi DM. Structure rhinoplasty: lessons learned in 30 years. Chicago: DMT Solutions; 2019

[89] Muhler G. Correction of the nose with autogenic rib cartilage transplantation. Hno 1969; 17: 294-298

[90] Walter C. Nasal reconstruction. The Laryngoscope 1975; 85: 1227-1240. doi:10.1288/00005537-197507000-00015

[91] Wee JH, Park MH, Oh S et al. Complications associated with autologous rib cartilage use in rhinoplasty: a meta-analysis. JAMA facial plastic surgery 2015; 17: 49-55. doi:10.1001/jamafacial.2014.914

[92] Dingman RO, Grabb WC. Costal cartilage homografts preserved by irradiation. Plast Reconstr Surg Transplant Bull 1961; 28: 562-567

[93] Kridel RW, Konior RJ. Irradiated cartilage grafts in the nose. A preliminary report. Arch Otolaryngol Head Neck Surg 1993; 119: 24-30. discussion 30-21

[94] Kridel RW, Ashoori F, Liu ES et al. Long-term use and follow-up of irradiated homologous costal cartilage grafts in the nose. Archives of facial plastic surgery 2009; 11: 378-394. doi:10.1001/archfacial.2009.91

[95] Kridel RW, Sturm AK. Dorsal Augmentation with Homologous Rib. Facial plastic surgery : FPS 2017; 33: 195-201. doi:10.1055/s-0037-1598031 
[96] Wee JH, Mun SJ, Na WS et al. Autologous vs. Irradiated Homologous Costal Cartilage as Graft Material in Rhinoplasty. JAMA facial plastic surgery 2017; 19: 183-188. doi:10.1001/jamafacial.2016.1776

[97] Erol 00. The Turkish delight: a pliable graft for rhinoplasty. Plastic and reconstructive surgery. 2000; 105: 2229-2241; discussion 2242-2223. doi:10.1097/00006534-200005000-00051

[98] Daniel RK, Calvert JW. Diced cartilage grafts in rhinoplasty surgery. Plastic and reconstructive surgery 2004; 113: 2156-2171. doi:10.1097/01.prs.0000122544.87086.b9

[99] Calvert JW, Brenner K, DaCosta-lyer M et al. Histological analysis of human diced cartilage grafts. Plastic and reconstructive surgery 2006; 118: 230-236. doi:10.1097/01.prs.0000220463.73865.78

[100] Aydinli S, Biskin S, Dinc AE et al. Bacterial cellulose as a new graft model for the Turkish delight technique in rhinoplasty: An experiment in 20 rats. Ear, nose, \& throat journal 2017; 96: E1-E5

[101] Erol OO. Long-Term Results and Refinement of the Turkish Delight Technique for Primary and Secondary Rhinoplasty: 25 Years of Experience. Plastic and reconstructive surgery 2016; 137: 423-437. doi:10.1097/01.prs.0000475755.71333.bf

[102] Tasman AJ. Dorsal Augmentation-Diced Cartilage Techniques: The Diced Cartilage Glue Graft. Facial plastic surgery: FPS 2017; 33: 179-188. doi:10.1055/s-0037-1598185

[103] El-Shazly M, El-Shafiey H. Soft versus hard implants in dorsal nasal augmentation: a comparative clinical study. Aesthetic plastic surgery 2012; 36: 1019-1027. doi:10.1007/s00266-012-9941-y

[104] Kreutzer C, Hoehne J, Gubisch W et al. Free Diced Cartilage: A New Application of Diced Cartilage Grafts in Primary and Secondary Rhinoplasty. Plastic and reconstructive surgery 2017; 140: 461-470. doi:10.1097/PRS.0000000000003622

[105] Muenker R. The bilateral conchal cartilage graft: a new technique in augmentation rhinoplasty. Aesthetic plastic surgery 1984; 8: 37-42

[106] Hafezi F, Bateni H, Naghibzadeh B et al. Diced ear cartilage with perichondrial attachment in rhinoplasty: a new concept. Aesthetic surgery journal 2012; 32: 825-832. doi:10.1177/109082 $0 \times 12455635$

[107] Lan MY, Park JP, Jang YJ. Donor site morbidities resulting from conchal cartilage harvesting in rhinoplasty. The Journal of laryngology and otology 2017; 131: 529-533. doi:10.1017/S0022215117000639

[108] Jurk V, Kampmann H, Iblher $\mathrm{N}$ et al. Long-Term Comparison of Rib and Ear Cartilage Grafts in Autologous and Allogenic Fascia Lata: An Experimental Study in a White Rabbit Model. Plastic and reconstructive surgery 2016; 137: 1465-1474. doi:10.1097/PRS.0000000000002133

[109] Ho TT, Sykes K, Kriet JD et al. Cartilage Graft Donor Site Morbidity following Rhinoplasty and Nasal Reconstruction. Craniomaxillofacial trauma \& reconstruction 2018; 11: 278-284. doi:10.1055/s-0037-1607065

[110] Denecke H], Meyer R. Plastic surgery of head and neck. Berlin, New York etc: Springer; 1967

[111] Straatsma CR. Surgery of the bony nose: comparative evaluation of chisel and saw technique. Plast Reconstr Surg Transplant Bull 1961; 28: $246-248$

[112] Rohrich RJ, Janis JE, Adams WP et al. An update on the lateral nasal osteotomy in rhinoplasty: an anatomic endoscopic comparison of the external versus the internal approach. Plastic and reconstructive surgery. 2003; 111: 2461-2462; discussion 2463. doi:10.1097/01. PRS.0000061005.27994.E3

[113] Gryskiewicz JM, Gryskiewicz KM. Nasal osteotomies: a clinical comparison of the perforating methods versus the continuous technique. Plastic and reconstructive surgery. 2004; 113: 1445-1456; discussion 1457-1448. doi:10.1097/01.prs.0000113031.67600.b9

[114] Robiony M, Toro C, Costa F et al. Piezosurgery: a new method for osteotomies in rhinoplasty. The Journal of craniofacial surgery 2007; 18: 1098-1100. doi:10.1097/scs.0b013e3180de6489
[115] Robiony M, Lazzarotto A, Nocini R et al. Piezosurgery: Ten Years' Experience of Percutaneous Osteotomies in Rhinoplasty. Journal of oral and maxillofacial surgery : official journal of the American Association of Oral and Maxillofacial Surgeons 2019; 77: 1237-1244. doi:10.1016/j.joms.2019.01.035

[116] Gerbault O, Daniel RK, Kosins AM. The Role of Piezoelectric Instrumentation in Rhinoplasty Surgery. Aesthetic surgery journal 2016; 36: 21-34. doi:10.1093/asj/sjv167

[117] San Nicolo M, Berghaus A. Advantages of piezoelectric technology in rhinoplasty. Hno 2019, doi:10.1007/s00106-019-0675-x.

[118] Kurt Yazar S, Serin M, Rakici IT et al. Comparison of piezosurgery, percutaneous osteotomy, and endonasal continuous osteotomy techniques with a caprine skull model. Journal of plastic, reconstructive \& aesthetic surgery: JPRAS 2019; 72: 107-113. doi:10.1016/j. bjps.2018.08.025

[119] Kocak I, Dogan R, Gokler O. A comparison of piezosurgery with conventional techniques for internal osteotomy. European archives of oto-rhino-laryngology: official journal of the European Federation of Oto-Rhino-Laryngological Societies (EUFOS): affiliated with the German Society for Oto-Rhino-Laryngology - Head and Neck Surgery 2017; 274: 2483-2491. doi:10.1007/s00405-017-4514-y

[120] Wähmann M, Riedel F, Kovacevic M et al. Comparison of piezoelectric and conventional osteotomy in rhinoplasty: A systematic review. Hno 2019; 67: 98-109 doi:10.1007/s00106-018-0606-2

[121] Goodale JL. A new method for the operative correction of exaggerated roman nose. Boston Med Surg | 1899; 140

[122] Barelli PA. Long term evaluation of „push down“ procedures. Rhinology 1975; 13: 25-32

[123] Saban Y, Braccini F, Polselli R. Rhinoplasty: morphodynamic anatomy of rhinoplasty. Interest of conservative rhinoplasty. Rev Laryngol Otol Rhinol (Bord) 2006; 127: 15-22

[124] Kosins AM, Daniel RK. Decision Making in Preservation Rhinoplasty: A 100 Case Series With One-Year Follow-Up. Aesthetic surgery journal 2019, doi:10.1093/asj/sjz107.

[125] Goksel A, Saban Y. Open Piezo Preservation Rhinoplasty: A Case Report of the New Rhinoplasty Approach. Facial plastic surgery : FPS 2019; 35: 113-118. doi:10.1055/s-0039-1678578

[126] Wähmann MS, Bulut OC, Bran GM et al. Systematic Review of Quality-of-Life Measurement After Aesthetic Rhinoplasty. Aesthetic plastic surgery 2018; 42: 1635-1647. doi:10.1007/s00266-0181199-6

[127] Devlin NJ, Appleby J, Buxton M. Getting the most out of PROMs: putting health outcomes at the heart of NHS decision-making. London: King's Fund; 2010

[128] Porter ME. What is value in health care? N Engl J Med 2010; 363 : 2477-2481. doi:10.1056/NEJMp1011024

[129] Ovretveit J, Zubkoff L, Nelson EC et al. Using patient-reported outcome measurement to improve patient care. Int J Qual Health Care 2017; 29: 874-879. doi:10.1093/intqhc/mzx108

[130] Nelson EC, Eftimovska E, Lind C et al. Patient reported outcome measures in practice. BMJ 2015; 350: g7818. doi:10.1136/bmj.g7818

[131] Staniszewska S, Haywood KL, Brett J et al. Patient and public involvement in patient-reported outcome measures: evolution not revolution. Patient 2012; 5: 79-87. doi:10.2165/11597150000000000-00000

[132] [Anonym] Guidance for Industry Patient-Reported Outcome Measures: Use in Medical Product Development to Support Labeling Claims. In: Administration USDoHaHSFaD; ed2009

[133] Basch E, Bennett AV. Patient-reported outcomes in clinical trials of rare diseases. J Gen Intern Med 2014; 29 (Suppl 3): S801-S803. doi:10.1007/s11606-014-2892-z 
[134] Calvert M, Kyte D, Mercieca-Bebber R et al. Guidelines for Inclusion of Patient-Reported Outcomes in Clinical Trial Protocols: The SPIRIT-PRO Extension. JAMA 2018; 319: 483-494. doi:10.1001/jama.2017.21903

[135] Alsarraf R, Larrabee WF Jr., Anderson S et al. Measuring cosmetic facial plastic surgery outcomes: a pilot study. Archives of facial plastic surgery 2001; 3: 198-201

[136] Meningaud JP, Lantieri L, Bertrand JC. Rhinoplasty: an outcome research. Plastic and reconstructive surgery 2008; 121: 251-257. doi:10.1097/01.prs.0000293866.57517.d4

[137] Saleh AM, Younes A, Friedman O. Cosmetics and function: quality-oflife changes after rhinoplasty surgery. The Laryngoscope 2012; 122: 254-259. doi:10.1002/lary.22390

[138] Bulut OC, Wallner F, Oladokun D et al. Long-term quality of life changes after primary septorhinoplasty. Quality of life research : an international journal of quality of life aspects of treatment, care and rehabilitation 2018; 27: 987-991. doi:10.1007/s11136-017-1761-8

[139] Klassen AF, Cano SJ, Scott A et al. Measuring patient-reported outcomes in facial aesthetic patients: development of the FACE-Q. Facial plastic surgery: FPS 2010; 26: 303-309. doi:10.1055/s-0030-1262313

[140] Schwitzer JA, Sher SR, Fan KL et al. Assessing Patient-Reported Satisfaction with Appearance and Quality of Life following Rhinoplasty Using the FACE-Q Appraisal Scales. Plastic and reconstructive surgery 2015; 135: 830e-837e. doi:10.1097/PRS.0000000000001159

[141] Ware J Jr., Kosinski M, Keller SD. A 12-Item Short-Form Health Survey: construction of scales and preliminary tests of reliability and validity. Med Care 1996; 34: 220-233

[142] Stewart MG, Witsell DL, Smith TL et al. Development and validation of the Nasal Obstruction Symptom Evaluation (NOSE) scale. Otolaryngology - head and neck surgery: official journal of American Academy of Otolaryngology-Head and Neck Surgery. 2004; 130: 157-163 doi:10.1016/j.otohns.2003.09.016

[143] Moubayed SP, Ioannidis JPA, Saltychev M et al. The 10-Item Standardized Cosmesis and Health Nasal Outcomes Survey (SCHNOS) for Functional and Cosmetic Rhinoplasty. JAMA facial plastic surgery 2018; 20: 37-42. doi:10.1001/jamafacial.2017.1083
[144] Bulut C, Wallner F, Plinkert PK et al. Development and validation of the Functional Rhinoplasty Outcome Inventory 17 (FROI-17). Rhinology 2014; 52: 315-319. doi:10.4193/Rhin13.098

[145] Klassen A, Jenkinson C, Fitzpatrick R et al. Patients' health related quality of life before and after aesthetic surgery. Br J Plast Surg 1996; 49: $433-438$

[146] Ribeiro RVE. Prevalence of Body Dysmorphic Disorder in Plastic Surgery and Dermatology Patients: A Systematic Review with Meta-Analysis. Aesthetic plastic surgery 2017; 41: 964-970. doi:10.1007/s00266-017-0869-0

[147] Picavet VA, Prokopakis EP, Gabriels L et al. High prevalence of body dysmorphic disorder symptoms in patients seeking rhinoplasty. Plastic and reconstructive surgery 2011; 128: 509-517. doi:10.1097| PRS.0b013e31821b631f

[148] Felix GA, de Brito MJ, Nahas FX et al. Patients with mild to moderate body dysmorphic disorder may benefit from rhinoplasty. Journal of plastic, reconstructive \& aesthetic surgery: JPRAS 2014; 67: 646-654. doi:10.1016/j.bjps.2014.01.002

[149] Bensoussan JC, Bolton MA, Pi S et al. Quality of life before and after cosmetic surgery. CNS Spectr 2014; 19: 282-292 doi:10.1017/ S1092852913000606

[150] Yang F, Liu Y, Xiao H et al. Evaluation of Preoperative and Postoperative Patient Satisfaction and Quality of Life in Patients Undergoing Rhinoplasty: A Systematic Review and Meta-Analysis. Plastic and reconstructive surgery 2018; 141: 603-611. doi:10.1097/ PRS.0000000000004102

[151] Sarwer DB, Gibbons LM, Magee L et al. A prospective, multi-site investigation of patient satisfaction and psychosocial status following cosmetic surgery. Aesthetic surgery journal 2005; 25: 263-269. doi:10.1016/j.asj.2005.03.009

[152] Arima LM, Velasco LC, Tiago RS. Crooked nose: outcome evaluations in rhinoplasty. Brazilian journal of otorhinolaryngology 2011; 77: 510-515

[153] van Zijl F, Mokkink LB, Haagsma JA et al. Evaluation of Measurement Properties of Patient-Reported Outcome Measures After Rhinoplasty: A Systematic Review. JAMA facial plastic surgery 2019; 21: 152-162. doi:10.1001/jamafacial.2018.1639 